\title{
Disabled By Nature? \\ The Care Economy: History, Critique, and Ethnography
}

\author{
by \\ Graham G. Macdonald
}

\begin{abstract}
A thesis submitted to the Faculty of Graduate and Postdoctoral Affairs in partial fulfillment of the requirements for the degree of

Master of Arts

in

Political Economy
\end{abstract}

Carleton University

Ottawa, Ontario

(C) 2014, Graham G. Macdonald 


\begin{abstract}
This thesis explores the relationship between the care economy and disability through both an ethnography of an assisted living program offered to students with disabilities living in residence at Carleton University, as well as through a theoretical and historical exploration of the relation of the care economy to human vulnerability. This thesis posits the interrelation of the constructions of disability and of care, and explores, analyzes and delineates the effects that this interrelation has upon care-workers and their clients. It engages and critiques the British Social Model theory of disability with theories of political economy and nature drawn from economic anthropology.
\end{abstract}




\section{Acknowledgements}

I would like to thank my supervisor, Susan Braedley, for believing in me and my work even when I did not, and for being critical, honest, and encouraging at every step. Thank you, to my family and my partner, for your unconditional support. I would also like to thank my friends, colleagues and professors at the Institute of Political Economy for being part of the supportive and dynamic environment that allowed me to produce this thesis. Finally, I would like to extend my sincere gratitude to everyone who participated in this study, and all the rest at Attendant Services, your thoughts and struggles have inspired this work, and as such it belongs to all of us. 


\section{Table of Contents}

$\begin{array}{ll}\text { Abstract } & \text { ii }\end{array}$

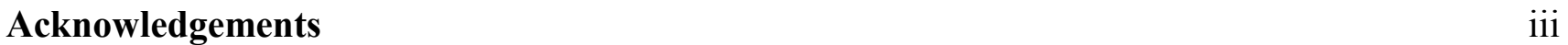

Chapter 1. INTRODUCTION 1

1.1 Care, Disability and Impairment $\quad 7$

1.2 The Disabling Environment: Nature, Culture and Impairment 11

1.3 The Importance of Disability as a Category of Political Economy 14

1.4 The Case Study: A Brief History of Attendant Services at Carleton University 17

\section{Chapter 2. Method and Methodology}

2.1 On History and Methodology 19

$\begin{array}{ll}\text { 2.2 Participant Observation } & 23\end{array}$

$\begin{array}{ll}2.3 \text { Interviews } & 24\end{array}$

FINDINGS: AN ETHNOGRAPHY OF INDEPENDENT LIVING

Chapter 3. "My Body is a Cage": Attending to Human Vulnerability 28

3.1 The Structure of Work in the Program $\quad 30$

3.2 A Gendered Workplace? $\quad 34$

3.3 Interlude: The Price of Being Unique 36

Chapter 4. Power and Policy: The Sinking Ship

4.1 Policy and Management in the Attendant-Client Relationship 43

4.2 "Telling a Dog Not to Bark" : The Short Arm of Policy 46

4.3 Together on a Sinking Ship: A Culture of Resistance Against Management 53

Chapter 5. The Mutual Experience of Disablement 
5.1 Neoliberal Training-Wheels or Emancipation? The Transition to Independent Living 57

5.2 Why Do We Care? The Attendant Experience of Disablement 61

5.3 Embracing Vulnerability: A Positive Culture 71

\section{Chapter 6. ANALYSIS}

6.1 The Myth of Independence or The Case for Social Equality

6.2 The Dialectic of the Care Worker

6.3 The Role of the Attendant Revisited: A Revised Workers Manual

6.4 "Independent Living is Dead, Long Live Independent Living"

\section{Chapter 7. A RETURN TO THEORY: Toward a Theory of Impairment}

7.1 Cutting the Gordian Knot: In Defence of a Social Model 92

7.2 The Crippled Savage I: The Founding of a Utopia Outside of Nature 99

7.3 The Crippled Savage II: The Question of Technology 103

$\begin{array}{ll}7.4 \text { Scarcity's Utopia } & 107\end{array}$

7.5 Scarcity and the Theory of Impairment 111

$\begin{array}{ll}\text { Chapter 8. CONCLUSION } & 117\end{array}$

$\begin{array}{ll}\text { References } & 124\end{array}$

\section{Appendix I: Ethics Protocol Components}

Sub-Appendix A: Informed Consent Forms for Interviews

Sub-Appendix B: Interviews Schedules for Attendants and Clients

Sub-Appendix C: Email Recruitment

Sub-Appendix D: Letter of Introduction

Sub-Appendix E: Ethics Protocol Application Form

Sub-Appendix F: Ethics Clearance Form 


\section{Chapter 1. INTRODUCTION}

How are autonomy, independence and care experienced in an Independent- or Assisted- Living setting, for both clients and their attendants, and do these experiences align with theories of disability? This thesis critically examines a program of Independent Living in order to assess how care and independence relate to one another for people with disabilities. Furthermore it looks at care as an economic process, a political relationship and a cultural construct. It brings theory and ethnographic findings into dialogue to critically inquire into the "nature" of disability and its relationship to care. This thesis presents a social theory of impairment in order to reconnect the notion of a socially constructed disability to the real material barriers and vulnerabilities with which care is concerned.

This ethnography is a study of Carleton University and Algonquin College's Attendant Services Program, a workplace of attendant care and independent or assisted living for people with disabilities living as students in residence on these campuses. Data was collected through participant observation working as an attendant at the Carleton University site and through semi-structured interviews with attendants and former attendants as well as clients and former clients of the program.

The research question is: are the distinctions between 1) able-bodied versus disabled, and 2) impairment versus disability tenable when one considers the role of political-economic relations of care in the construction of these categories? Furthermore, how does the lived experience of the relationship between attendants and clients reaffirm or undermine the validity of these existing categories?

While addressing questions raised by the literature of disability studies, political economy, and care ethics, this thesis is first and foremost a work in the discipline of economic anthropology because it rejects the separation of culture and economy from nature, as a theoretical and methodological starting point. The care economy is and has been a fundamental feature of every human culture and 
economy that has existed, in every kind of social organization from subsistence-based nomadic groups to commercial societies. This is because care economies address the basic natural fact of human vulnerability, and as such, care economies bridge the gap between natural and cultural processes. This thesis compares the case study to other economies of care in order to formulate a deeper analysis of disability, impairment and care in the present day political economy.

Mobility impairment due to congenital spinal cord conditions is the particular kind of disability represented in the research; these are incurable, permanent impairments, with a wide range of severity. This research examines the dynamics of everyday life for the clients and attendants involved in this program, and the tension between the "caregiving" done by the attendants and how the clients maintain their independence and autonomy. The Attendant Services Program follows the principle of "clientdirected care," meaning that clients are treated as the experts on their own bodies and needs; this is a principle that is meant to correct the paternalism, abuse and colonial power relations that characterized institutional care (Morris 1993). Jenny Morris posits four principles necessary to an emancipatory community care model:

"That all human life is of value; that anyone, whatever their impairment, is capable of exerting choices; that people who are disabled by society's reaction to physical, intellectual and sensory impairment and to emotional distress have the right to assert control over their lives; that disabled people have the right to participate fully in society" (Morris 1993, 21).

The Independent Living movement and other similar, disability rights-based initiatives have an uneasy relationship with care due to the reality that people with disabilities were and are incarcerated in "total institutions" which historically used the prerogative of care for the vulnerable to justify total control of their lives. ${ }^{1}$ While this is an important historical reality to keep in mind, it is not a starting

\footnotetext{
${ }^{1}$ Independent Living is usually more consumer-centered than the program at Carleton and Algonquin, and involves a greater degree of control by people with disabilities (Lord, 2010,13). Funding is usually given directly to them to arrange their care ( $\mathrm{COPOH}$ "defining the parameters of independent living"), rather than going through a management structure as it
} 
point for understanding what the process of care is and entails. As a process, care involves "caring about, i.e. recognizing a need for care; caring for, i.e. taking responsibility to meet that need; care giving, i.e. the actual physical work of providing care; and, finally, care receiving, i.e. the evaluation of how well the care provided had met the caring need" (Tronto, 2010: 160). Within each of these aspects of the process there are "many points where conflict, power relations, inconsistencies, competing purposes and divergent ideas about good care could affect care processes" (ibid).

This research, like the program it is studying, is rooted firmly in the practice of community care guided by Independent Living ${ }^{2}$; these programs are a work in progress, and need to be reflected upon and assessed by those within them and in a manner that is not concerned with streamlining or costcutting these services. There is much to be learned for universities who wish to operate a program such as the one at Carleton and Algonquin college, and, in spite of how proud this program is to be "one of a kind," there will likely be efforts by other universities to offer these programs and services. Since there is a lack of expertise on how to run this kind of program in practice, this ethnography will prove useful in providing an overview of the program and how it works at the ground level. The benefits and challenges of the program are not necessarily obvious, and there is much that needs to be understood in human rather than financial or administrative terms. To understand its complexities requires an in-depth

does at Carleton University and Algonquin College.

${ }^{2}$ The case study in question - the Attendant Services Program at Carleton University and Algonquin College - resembles more closely an "assisted living" program than an independent living one. Though this is the case, the program, and those within it, still tend to think of it and brand it as an independent living program. For this reason, the ethnography will refer to it as "independent living," because it is important to take the language and meanings of the subjects seriously in an ethnography - to use the language that they use. In technical terms, it may not be "independent living" per se, but in spirit, and in the minds of the subjects of this study, it mostly certainly is. "Independent Living" will be used throughout the ethnography in this sense to both represent a cultural ideal on one hand, and to denote broadly a certain kind of care-giving and care-receiving relationship in practice aimed at some permutation of that ideal. To call it simply "assisted living" does not capture the perceived relationship of the subjects in this study to an emancipatory social movement which is their ideal around or against which they orient their politics. The management, attendants and clients mostly think of it in terms of an independent living program, though acknowledge that there are different ways to do independent living. The program also sees itself as something of a transition to independent living from home-care, so as such it is not a "complete" reproduction of the independent living model, but it aspires to work in tandem with it and to perform a role within the broader project of independent living. (DeJong, 1979). 
look at relationships in the present, framed by the history of the material conditions of disability in capitalism, and the development of the concept of disability in modern thought.

This ethnography is informed by -and in dialogue with - literatures that continue to influence understandings of disability and care. The Social Model of Disability literature, particularly its central debates, is a starting point, as Independent Living as a concept relies upon many of its propositions.

This thesis begins with a literature review of the Social Model of Disability ${ }^{3}$ and critiques thereof, focusing on the debates around the conceptual separation of disability as a social construction and impairment as the material fact to which disability is anchored. This theorization of impairment versus disability is the starting point in most of the social model literature.

In section 1.1, this review looks at the work of Tom Shakespeare and Fiona Williams and their attempts to integrate the concept of care and the role of "able-bodied" within emancipatory approaches to disability, and the challenges they present to social model theorists. Section 1.2 puts theories of the social construction of nature in conversation with Tom Shakespeare's reformulation of disability and impairment, namely that the natural environment always already presents disability as an intrinsic limitation. The literature review closes with an historical look at disability as a category of political economy and the foundation of the welfare state in order to demonstrate how this relation between impairment and nature has shaped and been shaped by the industrial and modern world.

The second chapter, method and methodology, begins by situating this project as a work in historical materialism, using the ethnographic record from the discipline of economic anthropology to create a point of comparison for the care economy presented in this ethnography. It then delineates the method of Grounded Theory, through which the findings of the ethnography will be filtered to test existing theory and to build new theory. Section 2.1 describes the method of participant observation

${ }^{3}$ Primarily the British Social Model. 
used in constructing the ethnography, and section 2.2 covers the method and methodology behind the interviews.

The ethnography begins with a short introduction to the workplace in section 3.1, and continues in section 3.2 describing the basic structures, tasks, routines, layout and relationships within the workplace. In section 3.3, the gender dynamics of the workplace are described. The following section and closing part of the chapter presents a narrative of the daily tasks of the attendant and interaction with the clients, taken from the participant observation.

The fourth chapter continues deeper into the ethnography, looking at relationships of power, policy and management in the program. The first part of the chapter (4.1) puts on display the thoughts of the attendants and clients about the terms of their relationship with one another and who has the power within it. This is followed by a look at the role of policy in the workplace, specifically the attendant Code of Conduct, and goes on to describe how this is enforced and violated, paying particular attention to issues surrounding confidentiality in the workplace. The final section of the chapter (4.3) sketches a picture of how the program is managed, and details the sources of the existing culture of resistance by clients and attendants in the program against management.

The fifth chapter, and last descriptive section of the ethnography, details how attendants and clients together build a mutual experience of care and disability. The first section (5.1) presents how the program functions as a stage of transition for clients coming from home-care and going into more professionalized and formal care in the community. The following section (5.2) delves into how the attendants make a transition of their own in how they experience disability and in how their understanding of their role changes over time, through their engagement with the program. The final section (5.3) details how these two experiences converge to form a culture of disability and care, and specifically, a culture in which there is the potential to form a positive experience of disability. 
In the sixth chapter, the analysis, I put forth that independence is a myth, and that the best conditions for achieving functional independence for people with disabilities is through a relationship based on social equality and interdependence. The analysis supports the the claims made by Tom Shakespeare $(2000,2006)$ and Fiona Williams (2001) that interaction with other people through care is an aspect essential to understanding disablement. In section 6.2 , the analysis then examines the tension between the stated role of the attendant versus their performed role, and toward this end the section focuses on how the care worker experiences the alienation of their labour. The next section (6.3), incorporates both analysis and recommendation to realign a practical ethics of care to reflect the realities of alienation and marginalization faced by attendants. The analysis then shifts to bring the power of the program itself into view, examining the consequences for attendants and clients of the fact that this program is the only one of its kind.

Following this analysis of care and the social model of disability, the final chapter makes a return to theory in order to further engage with Shakespeare's critique of the social model as well as his theorization of impairment. Section 7.1 uses the literature on the human production of nature to address Shakespeare's argument on impairment, while 7.2 sets the stage for an empirical rather than abstract basis for a theory of impairment by addressing how scarcity is institutionalized in capitalism, versus other modes of production/social reproduction. Section 7.3 addresses the question of how technological progress figures in the creation of disability. The final parts, 7.4 and 7.5, explain the origins of the existing theory of impairment as a natural phenomenon. This final section explains that what is needed is not turning impairment from a natural theory into a social theory, but in understanding its function and origins as something which is already a social theory. The conclusion, section 8 , brings the findings, analysis and theory together to put this new understanding of impairment into conversation with the dynamics of the attendant-client relationship as well as its place in the broader political economy. 


\section{LITERATURE REVIEW}

\subsection{Care, Disability and Impairment}

This thesis engages with the political debates on which approach to understanding disability offers the most viable path toward a better world for people with disabilities. The following literature review gives a brief background on these approaches and the central debates around which they orbit. In starting with the literature, it will establish a sense of the broader significance of the research presented in this thesis and allow the questions raised in these debates in the literature to better frame the findings as they are unfolded in the ethnography.

The British social model of disability, pioneered by the likes of Paul Hunt (1976), Vic Finklestein (1981), Michael Oliver and Colin Barnes (2012), and UPIAS (1976), among others, has maintained that impairment is the mean fact of a physical impediment, and disability derives from how impairment is treated and perceived in the social, cultural, policy-related, and political-economic contexts (Shakespeare 2006). These authors' various perspectives have developed a family of social models of disability, and have given rise to the insight that disability is not located in an individual's 'biological inferiority', but in practices and ideas which build social and material environments that are hostile or indifferent to impairment. Thus, environments create disability in those they exclude. Indeed, "at the level of the experience of people with disabilities there is a range of disadvantages and limitations that have everything to do with how people react - their attitudes and behaviours - and nothing to do with one's incapabilities" (Bickenbach 1993:136-137). This perspective has, historically, been an indispensable tool for disability rights activists, but, as criticized by Shakespeare, has ossified into something of a blunt instrument because the rigidity of this distinction does not allow for a more 
nuanced politics (Shakespeare 2013). The social model also "assumes what it needs to prove: that disabled people are oppressed" which, in turn, bears the assumption that disability not only involves oppression, but is oppression by definition (Shakespeare 2013: 218).

Tom Shakespeare, a well-acknowledged critic of the social model of disability, argues that “disabled people face both discrimination and intrinsic limitation... even if social barriers are removed, it will remain disadvantageous to have many forms of impairment" (Shakespeare 2013, 220). Shelley Tremain (2002) has also claimed that the social model conceptualizes impairment as though it were a universal concept that exists before or outside of the realm of social meaning. The trouble with the hard-and-fast divide between impairment and disability is that impairment itself "cannot be understood as an abstract category, since it always and only occurs in a particular social and historical context which determines its nature"(Abberly 1996, 61).

The social model of disability is and has been an invaluable theorization of disability, and is a pillar of this thesis, but as such will be subject to critique in what follows. The main goal of my critique is to uncover the basis of the theory of impairment by reconnecting the social model of disability with the intrinsic material barriers with which care grapples. The everyday lives of people involved in attendant care are examples of the ways in which the social whole operates to cope with the material and physical implications of disability. Since disability is a relational concept, meaning it is defined not by absolute standards, but by a person's relationship to a material and social environment, then it follows that the relationships of impaired people to their attendants (as their means of dealing with their material environment) are a starting point in discovering what disability/impairment is and entails, and to investigate Shakespeare's claims that there is more going on than simply oppression.

The relationship between care and disability is a much contested one. There are ongoing debates in the disability studies literature about the place of care within the discourse of disability liberation: 
some seem to think there is scant place for it in a political movement which asserts independence and autonomy (Oliver and Barnes 2012, 1996), while others present care as a necessary element in the struggle for rights and autonomy (Shakespeare 2006, 2013). In a related but separate literature on care, however, caring is presented as an ethical framework challenging the notion that anyone can be an autonomous and self-sufficient human being (Tronto 1993, F. Williams 2001). Fiona Williams, in response to critiques of care ethics by disability scholars, concedes that without the structures and conditions for equality, it becomes incumbent on the cared-for person to ensure that the carer behaves virtuously, and thus places moral onus on the cared-for person (F. Williams 2001:479).

In the disability rights discourse, the need for independence has overshadowed care due to the historical fact of a very illiberal and paternalistic institutional care regime. The political strategy of disability rights has largely been written care out of the discussion, though "in the longer term, disabled people's... interests would be better served by a society that valued interdependence and acknowledged the vulnerability of all, [but] the immediate strategy for disabled people is for rights to secure the conditions of independence" (F. Williams 2001:481). Though much of the disability studies literature and the care ethic literature seem to agree in principle and put great emphasis on the interdependent nature of human life, the rights-based approach to disability has often disavowed care in its political program. Disability does often require care, and this is unavoidable; but in making the political distinction between impairment and disability, care became one of the social factors seen to create disability out of impairment.

Independent Living, as a movement and in programs, runs the risk of marginalizing care and care workers as a result of protecting the very independence for people with disabilities that it aims to produce. Despite endorsing independence in a qualified form, as functional autonomy and selfdetermination, Independent Living marginalizes the care-workers it employs. This marginalization 
comes from seeing the interests of care workers as antagonistic to the interests of disabled people. Furthermore, both paid and unpaid care workers are generally women, and often racialized, making the already diminished value of the work even less visible by tying its value to hierarchies of gender and race. Marginalization of care-workers can often translate into worse conditions and quality of care for recipients of care. Independence has its logical limits when it is being facilitated by another person, therefore the living conditions of persons with disabilities who receive care are inextricably tied to the conditions of those who provide it.

Care, though instrumental to the actual practice of Independent Living, is often kept in the shadows by the strong emphasis that the political rhetoric of Independent Living puts on independence. As Tronto argues, "a political order that presumes only independence and autonomy as the nature of human life thereby misses a great deal of human experience and must somehow hide this point elsewhere," for example in the rigid separation of 'public' and 'private' life (Tronto, 1993: 135). The separation of the public and the private may not be altogether present in Independent Living arrangements; what people with severe physical disabilities experience as private or public may be radically different than the standard conception, especially for a person with a severe physical disability who requires assistance for a wide array of tasks and routines. The question of how, or if, one can maintain a degree of separation between their public and private lives in an Independent Living setting will be an important part of this inquiry. Mapping this boundary is one way in which this thesis reintegrates care into the debate on whether impairment and disability can be separately conceived. The work of carers also gets caught up in this separation between public and private, and is integrated into the "private sphere" of life, which is generally underpaid/unpaid "women's work" (Armstrong, 1997: $37-61)$. 


\subsection{The Disabling Environment: Nature, Culture and Impairment}

When the disabling environment is a natural one, a major fault line in the impairment/disability debate opens. Here, the literature review introduces critical theories of nature which the thesis will integrate to better address the questions raised in the debates that occur in the disability studies literature.

Tom Shakespeare, in his critique of the social model notes that despite efforts to promote universal access socially and materially, "numerous parts of the natural world will remain inaccessible to many disabled people: mountains, bogs, beaches are almost impossible for wheelchair users to traverse" (Shakespeare 2013: 219). This may seem an obvious argument, but it raises a naive question about the nature of impairment that demands an answer and a deeper exploration. One may extrapolate that indeed, impairment is what happens in natural conditions where geography is not manipulated to serve human ends: a state of nature, as Shakespeare seems to posit. A social model approach would contend, and fairly, that:

"Human beings are, in common with other primates, social animals. We have neither the physical ability nor intellectual capacity [one may well add emotional/mental ability] to survive as truly independent, isolated individuals. Hence as in all previous societies, we live in a state of mutual interdependence... the dependence of disabled people is not a feature which marks them out as different in kind from the rest of the population, but is different only in degree" (Oliver \& Barnes, 2012: 127).

How human beings interact with their environment is then better conceived as a question of social groups working with material resources to provide for a whole, rather than individuals working with their own innate physical capacities to provide for themselves. Proponents of Care Ethics make similar arguments about the social basis of addressing material problems (Williams, 2001). 
Though adapting to living within nature's barriers may seem better accounted for by looking at the group rather than individual, it does not mean that it cannot be understood on the individual, physical level, where impairment may present serious difficulties and exist as a material reality. What about mountains and bogs, are they social constructions as well? To answer that question in the affirmative would be to elide the experience of impairment and disability, relegating it to exist only as an oppressed and marginal identity. The diversity and importance of the human experience of the limitations of the body are undersold in this narrow view because

"Everyone is virtually disabled, both in the sense that able-bodied norms are "intrinsically impossible to embody" fully, and in the sense that able-bodied status is always temporary, disability being the one identity category that all people will embody if they live long enough" (McRuer 2013: 374).

McRuer's assertion is borne out by the fact that "in countries with life expectancies over 70 years, people spend an average of 8 years living with disabilities" (UNENABLE, Factsheet, 2011) not to mention the vulnerability of human beings in infancy and early childhood. What is so powerful about the category of disability is that it presents the human body and identity both as malleable (Davis 2013:273) and, I might add, demonstrates just how tied we as human beings are to our material environment.

In trying to address the material nature of disability within the social model, the "social creationist" view of disability, endorsed by Oliver and Barnes (and Paul Higgins (1996), among others), holds that ideas are material forces and not simply free-floating. This perspective looks towards combating institutional discrimination, which has material consequences, rather than towards changing "social attitudes" which social constructionists may advocate (Oliver and Barnes 2012: 125-126). This thesis takes this approach a step further.

In this thesis, my goal is to examine not just how ideas are material forces in creating disability, 
but how conceptions of material forces themselves create impairment. What I wish to put on display is that material forces are represented by ideas which hide as much as they may reveal about reality. To support this end I will examine how the concept of nature, as it has been so carefully analyzed by second-wave geographers, ecological historians, and economic anthropologists, as an ideological construct (Smith 1984, R. Williams 1980, Braun 2002, Braun and Castree 2001, Cronon 1979, Young 1973), helps to get beyond the disability/impairment distinction. Insofar as this thesis is trying to contribute to complex theoretical and political debates, it aims to do so by asking naive questions about disability, impairment and the environment.

This project recognizes that "impairment is not a 'natural' but an historically changing category; equally not all restrictions on human activity are oppression" (Abberly 1996, 64). This thesis does not aim to make impairment into an abstract category of nature, but rather to place the concept of nature in a historical perspective in order to understand the very limitations of impairment acting as a universal, abstract category of nature. The discipline of political ecology offers a view of nature that is dynamic, social and historical. The insights of this field stem from the methodological basis that human beings are always already a part of nature, and that the "physical constraints and opportunities nature presents societies with can only be defined relative to specific sets of economic, cultural and technical capacities" (Castree 2001: 13).

An ecological understanding of disability/impairment is not an unprecedented theoretical step. Writing from a political economy perspective, Gary Albrecht (1992) puts forth an "ecological model of disability patterns in a society" based on a physical environment (food, technologies, natural resources, climate, energy) as the container for the biological environment (disease patterns, pathogens, viral and bacterial agents) that also provides the context for the cultural environment (means of production, social organization, economy, ideologies, lifestyles, level of technology, biogenetic characteristics). 
Albrecht maintains that "the concept of a disabling environment ... forces us to acknowledge that disabilities are physically based but socially constructed. Societies then produce disabilities different from impairments." (Albrecht 1992: 34-35). This thesis, then, will build upon this foundation to see what the everyday lives of people with severe disabilities reveal about that difference between disability and impairment. Furthermore this thesis aims to develop an understanding of how the social and natural environments overlap to become "disabling."

Furthermore, the aspect of care shows how human beings cope with impairment in their social orders, as well as how they socially construct, deconstruct and reconstruct meanings around disablement. The line between having functional independence and being cared for is a blurry one, as Jenny Morris writes

"in the context of the economic inequality that accompanies significant physical impairment in industrialized societies, the need for personal assistance translates into a need for 'care' in the sense of a need to be looked after. Once personal assistance is seen as care then the carer, whether professional or a relative, becomes the person in charge, the person in control. The disabled person is seen as being dependent on the carer, and incapable of even taking charge of the personal assistance s/he requires" (Morris, 1993: 23).

There is a conflict with clients' autonomy, then, if there is an overbearing emphasis on "care" by the attendants. This research examines, through participant observation and interviews, how this tension plays out in the relationships taking place within the Attendant Services Program.

\subsection{The Importance of Disability as a Category of Political Economy}

This section of the literature review addresses disability's role, or more accurately, lack of a role in modern society, and how it has been shaped and understood through the discipline of political economy. As a researcher trained in this discipline, political economic processes and relationships are 
central to my understanding and analysis of social phenomena. Moreover, disability is very important to a deeper understanding of political economy because it is largely a "repressed" category that poses perennial and intractable problems to an economistic worldview. This thesis challenges the notion that disability can be narrowly conceived as a state of economic dependency, and expands its meaning as a political-economic category.

Disability is a modern concept, emerging as a focus of social policy during the upheaval of the Industrial Revolution, which created large groups of "dependents" -- or people without a means of economic support in eighteenth to nineteenth century Britain (Oliver and Barnes 2012: 83). The English Poor Laws were the legislative mechanism drafted to ameliorate this situation. These laws provided the state with tools to distinguish from the "deserving" and "undeserving" poor in order to administer welfare where it was most needed. The line between deserving and undeserving poor was meant to distinguish between those who legitimately could not work, and those who could work but did not.

Since the Industrial Revolution "work has been organized around the twin principles of competition between individual workers and maximization of profit. Inevitably disabled people have suffered because of the way work has been organized around those two principles" (Oliver 1996: 33). Disability/impairment, to functionaries in the welfare bureaucracy, represents an inability to work or to produce that is to be met with some form of welfare provision.

People with disabilities were among those thrown into economic need because of their inabilities to adapt to the changing conditions of work. The "speed of factory work, the enforced discipline, the time-keeping and production norms - all these were highly unfavourable changes from the slower, more self-determined methods of work into which many handicapped people had been integrated" (Ryan and Thomas 1980: 101). The purpose of the welfare state was to provide for those 
who could not sell their labour as a commodity on the competitive and individualized wage labour market. Acting as the provider, the welfare state had the authority to make the distinction between those who could work and those who could not and would therefore receive state help. The welfare bureaucracy and the medical profession worked in tandem to devise more scientific and exacting criteria for making the distinction between "deserving" and "undeserving" poor.

Disability is one aspect in society which is seen, almost categorically in many industrialized countries, to warrant state intervention into the lives of individuals. As D.J. Brewer expressed in 1875,

"It is, strictly speaking, the pauper, and not the poor man, who has claims on public charity. It is not one who is in want merely, but one who, being in want, in unable to remove or prevent such want. There is the idea of helplessness as well as of destitution. We speak of those whom society must aid, as the dependent classes, not simply because they do depend on society, but because they cannot otherwise than thus depend. Cold and harsh as the statement may seem, it is nevertheless true, that the obligation of the state to help, is limited to those who are unable to help themselves" (Brewer, 1875: 74-75)

These policy interventions have varied widely between providing things like disability pensions and accessible architecture, to institutionalization and eugenics programs.

Disability is at the heart of the modern welfare state. It raises perennial questions of how a state takes care of its own citizens and redistributes wealth and resources to that end. Disabled people are often the "poorest of the poor" within their societies, given their relative exclusion from the labour market (World Bank, 2008). To put things in perspective, around half of the world's impairment is linked directly to poverty (DFID 2000, 2011 cited in Oliver and Barnes 2012), so it should be understood that poverty gives rise to impairment, and simultaneously disability impoverishes. A central problem of the welfare state in addressing this need has been that "the work-based model of social membership and identity is linked to the prevention/cure-oriented perspective of allopathic medicine and ... involves a value judgement on the undesirability of impaired modes of being" (Abberly 1996, 
74), making impairment a problem to be solved rather than a consequence of human diversity. This kind of instrumental thinking treats people as objects whose main purpose is to work and reproduce workers.

Political economy, as a mode of enquiry, is indispensable in "constructing a theory of disability oppression because poverty and powerlessness are cornerstones of the dependency people with disabilities experience" (Charlton 1998: 23). The attendant-client relationship within the Independent Living programs is the latest chapter in this political economy of disability and care, which provides care on the premise that disability is a product of social and environmental conditions. Therefore, it is with this political economic relationship that the analysis will begin.

\subsection{The Case Study: A Brief History of Attendant Services at Carleton University}

The Attendant Services Program at Carleton University began with limited services in 1986; prior to this all students with disabilities lived on one floor of one residence house and received care from the nurse who was on morning duty at the residences. Throughout the rest of the day they had to remain independent or get assistance from their floor-mates. The nurse was responsible for the entire residence population, but ended up spending most of her time helping a few students with disabilities. Beginning in 1986, two local health care companies started providing service in residence, and most of the attendants staffing this program were drawn from the residence population. The primary responsibilities of the attendants were to assist students with disabilities with morning and evening care, allowing the nurse to return to regular duties. Outside of morning and evening routines, students with disabilities were without access to help for most of the day. At this point, only students with minor disabilities could live in residence because the attendants were largely untrained, had little policy to 
follow, and were underpaid. This service was funded through a small grant from Vocational Rehabilitation Services (VRS), an organization that had never funded this kind of arrangement before. Although the program showed potential, the limited capacity of the VRS made an increase in funding unlikely.

In 1986, the director of the Paul Menton Centre for Students with Disabilities applied to the Ontario Ministry of Health for a grant that would allow the program to operate 24 hours a day. The two health care service providers that had been running the program previously both sought exclusivity over the management of the expanded services. Both were, however, cut out of the deal when the university decided it would run the program itself, in order to side-step paying off-site management in a move to ensure that attendants would be paid more. In September of 1987, the new 24 hour service began, with little formality and training, but with goodwill and a strong commitment to the principles of Independent Living. Throughout the 1990's and 2000's, new residence buildings were built with a focus on accessibility, and linked to the underground tunnel system which had unintentionally made the Carleton Campus one of the most wheelchair accessible in the country. By 1990, the program's service was extended to operate through the summer, making its operation year-round rather than simply for the duration of the eight month academic year. (Paul Menton Center 2006). This prevented students with disabilities from having to struggle to find accessible housing for the summer, which was (and still is) in extremely scarce supply.

In 2001 the program expanded to the Algonquin College residence, making Algonquin College the first Canadian college to offer 24 hour attendant services. The program has been growing steadily since then and remains reliant on grants from the Ontario Ministry of Health and Long Term Care in 
order to maintain its funding. ${ }^{4}$

\section{Chapter 2. METHOD AND METHODOLOGY}

\subsection{On History and Methodology}

Drawing on Political Economy as an analytic lens, this project's methodology seeks to weave a longue duree history into an understanding of the practices of a specific culture in the present. The history in question is of the relation between people with impairments, their paid or unpaid helpers, and the political economic structures that broadly shape the Attendant Services Program at Carleton University and Algonquin College. This thesis derives from an understanding of changing modes of production and social reproduction through historical materialist political economy. My enquiry is in the spirit and method of the French school of economic anthropology (Hann \& Hart, $48-53$ ) and my understanding of history is deeply indebted to Walter Benjamin's On the Concept of History (1940).

The ethnographic element is absolutely necessary and central to this enquiry because the actual patterns and rhythms of social life often escape the bird's eye view of theory and history. Ethnographic evidence must be brought back in to the discussion as the particular through which we can address the more universal questions of theory. The purpose of this enquiry is to put disability theory to the test, and to build new theory by treating disability culture not as a representation of dominant ideology, but as human praxis (Willis, 1977: 3). This ethnography is the starting point for theory, and not the other

\footnotetext{
${ }^{4}$ The exact amount provided by these grants, and the budget for the program itself was not disclosed to the researcher or anyone known to him during the study, despite multiple attempts to gather this information from the Ministry of Health and Long Term Care, the Champlain Community Care Access Centre, and the management of the Attendant Services Program.
} 
way around; while it is impossible to fully weed out or even trace the origins of exogenous ideas about disability that manifest in this thesis, care has been taken to maintain a reflexive understanding of my own personal and intellectual place in the research (Rose, 1997: 311). A very important aspect of this "reflexivity" is to keep a priori theoretical categories at arms length in interpreting results (Emerson, Fretz \& Shaw, 2010: 132). In order to do this, the methodological framework that I have adopted is the "grounded theory" approach, which is inductive and qualitative (Corbin \& Strauss, 1997). Grounded theory is useful for building theory from the ground up, so to speak, and involves using ethnographic data to verify or alter existing theory.

Participant observation, interviews with attendants and clients of the Attendants Services Program and a content analysis of this program's key policy documents are the methods which will bring together history, critique and ethnography in this thesis. In framing and interpreting the data from the interviews, participant observations and policy documents, this critical insight into the function of rules within social orders has been instrumental:

Every order of explicit social normativity has to rely on a complex network of informal rules which tell us how we are to relate to the explicit rules. How are we to apply them? To what extent are we to take them literally? How then, are we allowed, solicited even, to disregard them? To know the meta-rules of a society is to know the unwritten rules of how to apply its explicit rules. When to use them or not to use them, when to violate them, when not to use a choice that is offered. For example, often we are obliged to do something but we have to pretend that we are doing it as a free choice. Recall the polite offer meant to be refused: it is a habit to refuse such an offer, anyone who accepts it has made a vulgar blunder. The same goes for many political situations in which a choice is given on condition that you make the right choice. You are solemnly reminded that you can say no, but that you are expected to say yes. (Zizek, 2006)

Thus while it is very important to talk about how the rules are followed within a social order, it is perhaps more significant to be aware of the acceptable ways in which these rules may be broken, and how choices presented are not meaningful or "free" choices at all. 
If politics is the realm of the possible, and explicit rules do not place absolute constraints on what is possible, then we do not yet know what is possible if explicit rules are taken at face value. Without this insight, the attendant services workplace would remain a black box, filled only with baffling irrationality. The only method for understanding the workings of a social order is to participate in it and gain knowledge of its history. Knowing a history, and participating in the present, however, are not wholly distinct things, because in order to participate in a social order, one tacitly and necessarily internalizes a version of its history.

History is embedded and ever-present in the ways in which people communicate and relate to one another. Relationships, and the patterns and scripts they follow, are formed through history, not outside or parallel to it. The history of social orders is moved by changes in relationships. In the case of this research, that would include relationships between genders; between human beings and their environment; between the disabled and the non-disabled and so on. These relationships are political, in that their terms establish the realm of possibility for social interaction between parties. They are also economic in that the "possibilities" for each group represent their range of options to achieve subsistence and livelihood within a given social order. Finally, relationships in social orders, as well as being political and economic, are undoubtedly also cultural. Here, again we take the word "culture" to mean "human practice"; because in order to maintain a certain pattern of political and economic relationships there are necessarily a wide range of practices, both material and social, that are involved in creating meaning for the participants.

As Blaney and Inayatullah maintain, "to give in to the cultural is to be newly sensitive to cultural variation, thereby downgrading economics from its status as natural law. It is to confront different meanings and purposes than those central to contemporary economic analysis, transforming economics into a branch of ethnography" (Blaney and Inayatullah, 2010: 19-20). As such, this 
ethnography follows the methodological leanings of Blaney and Inayatullah in that it seeks to interpret a social organization, the Attendant Services Program and the broader political economy of disability that it represents, as a cultural project. Seeing it through this lens then, nothing is taken for granted as natural, but instead treated as a human project with its own peculiar meanings and purposes. The ethnography treats the Attendant Service Program at Carleton University and Algonquin College as one such cultural artifact that can tell us a great deal about the broader cultural meaning of the political economy of disability.

Following Blaney and Inayatullah, the analysis and theory built from this ethnography explores the utopian visions of this Program, relating them to the "savage" condition to which the client-directed care model implicitly defines itself against. This "savage condition" can be properly understood as a "state of nature" in the Enlightenment sense of representing a kind of original sin. The current social order imagines itself as having evolved away from this barbarous state - the question to ask here is why does it believe this, and what possibilities and horizons does this present? And which does it preclude?

A vital methodological insight about culture and history comes from the German philosopher Walter Benjamin:

"There has never been a document of culture, which is not simultaneously one of barbarism. And just as it is itself not free from barbarism, neither is it free from the process of transmission, in which it falls from one set of hands into another. The historical materialist thus moves as far away from this as measurably possible. He regards it as his task to brush history against the grain.” (Benjamin, 1940, vii).

What is considered "culture" in one place and time may well be interpreted as barbarity from another standpoint. In the historical materialist tradition, there is no place here for a history that sees itself as a line of technical and human progress marching across the blank page of time. The line between culture and barbarity is at best an artificial one.

Benjamin explains "The tradition of the oppressed teaches us that the "emergency situation" in 
which we live is the rule. We must arrive at a concept of history which corresponds to this" (ibid, viii). Blaney and Inayatullah (2010) describe the "Wound of Wealth" as that which the linear and progressive vision of history is forever promising to bandage, but perennially fails to remedy. Central to this idea is the notion that the benefits of wealth and progress are created and distributed in such a way in society so that they actually produce poverty and misery as never before seen. The "Wound of Wealth" that disability represents for modern liberal capitalism will not be treated so naively as to garner "astonishment that the things we are experiencing in the $20^{\text {th }}$ [and 21st] century are "still" possible. This] is by no means philosophical. It is not the beginning of knowledge, unless it would be the knowledge that the conception of history on which it rests is untenable" (Benjamin, 1940, viii). In order to arrive at a historical materialist understanding of disability, the care economy must be addressed in its role of perpetually attempting to bandage this "Wound of Wealth." Care deals with the consequences of the "emergency situation" of the rejected and excluded, "defective" people who are dragged along by the rising tide of modern progress.

In the context of a changing political economy, the terms upon which care is both given and received shape the power dynamics within the care economy. This research treats care as its category of theoretical and practical importance, and as such, critically examines its role in constructing the social meaning and conditions of disability. Care, which is fundamentally about attending to the disadvantaged in situations of inequality (Williams 2001), can also perpetuate and service those unequal arrangements.

\subsection{Participant Observation:}

In my research, I acted as a participant observer, working for 150 hours over three months as an 
attendant in the Attendant Services Program at Carleton University. This research developed from the experience that I acquired during two years of work as an attendant, during which I developed an understanding of what it is like to be involved in an attendant service workplace. During the three months of participant observation, I did the job of an attendant and then reflected critically on the day's work and interactions through constructing fieldnotes. This reflection is critical in that it attempts to not take for granted anything that goes on, including things that may seem commonplace or mundane. The truly critical aspect of the reflection on the participant observation is in deciding what to mention.

The gift of the ethnographic method is that even though I had already been intimately involved in the workplace, the ethnography allowed me to both step further inside but also, crucially, take a step back and see the relationships and everyday tasks and interactions from a much different angle than I had previously. The field-notes were careful not to impose outside meanings or categories, but rather to work within the categories used by those involved in the program and to appreciate them on those terms, first and foremost (Matza, 1969: 15 - 40). The categories of the greatest importance in this study are "attendants," "clients," and "management"; these three categories were even more defining than the disabled/able-bodied categories within the culture of the workplace. Since this study analyzes the structure of social relations at Attendant Services, these categories are treated as roles seen to influence the behaviours of their occupants regardless of their temperament, origins or personality (Gluckman, 2009). Participant observation revealed aspects of experience I had taken for granted, even as someone whom was aware of disability issues and had experience working as an attendant.

\subsection{Interviews:}

The interviews focused on how clients and attendants perceive themselves in relation to one 
another in order to get a sense of the relationships taking place under the rubric of Independent/assisted Living. The dynamics of the care relationship are subsumed under the banner of "service" and the person with a disability is not a dependent ward in an institution, but the client of the attendants, who are working in the clients' homes. The interviews explored how both the attendants and clients conceptualize their role and position, and how both deal with the kind of cages that these scripts create. Furthermore, the interviews examined the inner workings of how attendants and clients work towards the shared goal of the client exercising functional independence through the support of the attendant.

Eleven interviews were done over the period of two months, with five attendants/former attendants and six clients/former clients. Five interviewees were female and six were male. The interviews were instrumental in deepening and broadening understanding and analysis, both complementing and informing participant observation components, and allowing for data triangulation, thus improving the validity of the findings (Emerson, Fretz and Shaw, 2011). The participants for interviews were recruited through personal contact. They were audio-recorded and transcribed, and the transcriptions, together with the fieldnotes, were coded for themes. Interviews were carried out at the beginning of the period of participant observation and throughout the rest of the study.

As participant observation deepened my understanding of my own experience and prompted new thoughts and thought processes, the interviews allowed me to tap into other attendants' experiences and thoughts as well as the experiences, concerns and thoughts of the clients of the program. ${ }^{5}$ The attendants were asked about their prior experience with disability and how their understanding of disability had evolved throughout the course of their work as an attendant. They were also asked about how they characterized their relationship with clients, and any inequalities of power they felt were at play in that relationship or in the workplace at large. The interviews with attendants also touched on the

\footnotetext{
${ }^{5}$ The informed consent form, the interview schedules and ethics review are all included in appendix I.
} 
idea of dependency as an aspect of power relations, asking whether they felt the clients were dependent upon them, or whether they felt dependent upon the clients. The interview also focused on how they saw themselves as workers and how they thought and felt about the work they were doing.

The interviews with the clients focused on their experiences with the attendant services program at Carleton, though allowing for a comparative perspective with home-care (by family) and other, differently run and administered attendant service providers. The interviews addressed questions of how the interviewees' impairments impacted their daily lives and whether physical or social barriers posed greater challenge to their well-being. As with the attendants, the dynamics of power in the attendant care workplace were discussed at length, including issues of dependency, interdependence and independence. The central aspect discussed was the relationships clients had with their attendants, and how they would characterize those relationships and how they impacted their well-being, independence, privacy, dignity as well as the quality of their care and the level of professionalism involved therein. More broad and general views on and experiences with disability were addressed, such as whether or not the interviewees felt their disability had a positive impact on their lives and if they believed their impairment to be a "natural" thing.

The interviews were all very conversational, comfortable and casual, with everyone involved appearing happy to participate and give their thoughts. Significantly, the most unanticipated and surprising insights were gained through interviews with the attendants. This can be attributed to the following: the participant observation, due to the nature of the job being that attendants work in parallel with each other and directly with the clients, revealed an active understanding of the clients' experience while the experiences of the attendants remained largely hidden from my view. My breadth of understanding from interacting with many different clients was thus broader, deeper and more varied; conversations such as the ones that took place in a structured way in the interviews were actually 
relatively commonplace in my interactions with the clients, to the extent that in the interviews, we were re-hashing previous discussions we had had.

With attendants, this was not the case. The "water-cooler" discussion that took place during down-time in our office provided discussion which was much less explicitly about disability; the conversation was often not representative of the political views of attendants on disability, but rather more reflective of a need to deal with stresses of attendant work with others who understood. The stress of being an attendant was apparent from the participant observation, but the political, social and personal sentiments underlying that stress remained a black box until the interviews were conducted. Interviews with the clients also held some valuable insights about the experience of attendants because every client has been involved with a far greater number attendants in their lives than I could have hoped to interview. Those experiences and stories were also vital in developing an understanding of the dynamics of attendant work.

Another reason that the attendant interviews revealed more surprising findings were biases I had inherited from a number of sources, some of which are difficult to pin down. Having internalized the accounts of generations of disability literature, I was more concentrated on the experiences of people with disabilities rather than those of their attendants. This is interesting because in my first year of work as an attendant, I was acutely aware of the labour issues involved in being an attendant and privy to a more holistic view of interdependence between attendants and clients. This "organic" understanding did not change so much as it was pushed into the background after my reading of disability studies literature. In conducting this research it has become apparent that there are relationships and ontologies that have come into being since the disability rights movement began and that deserve to be elaborated upon. 


\section{FINDINGS: AN ETHNOGRAPHY OF INDEPENDENT LIVING}

\section{Chapter 3. "MY BODY IS A CAGE" : ATTENDING TO HUMAN VULNERABILITY}

The thing I remember about my very first shift as an attendant was the anxiety of not knowing what the interaction would be like, whether it would be strained or natural, the kind of personality I would have to deal with, and what they might ask me to do. I was there as an observer for a few shifts, shadowing a senior staff attendant as he went through the motions of the morning routines: getting people out of bed, helping them to shower, brush their teeth and go to the bathroom and then helping them get dressed so they could go to early classes without delay. I was, as all trainees, a voyeur to a standard weekday morning of the clients of attendant services. This is how attendants train, by observing and being observed. Since the "care" is client-directed, there are actually very few technical aspects of the job which require intensive training. Learning how to interact with people with disabilities in their homes and learning the social role of the attendant is the implicit goal of the training.

My training was, more accurately, my introduction to people with disabilities. I had not known anyone with a severe disability in my life before and frankly did not know how to approach disability in general. I remember the initial anxiety before what was literally my first wake-up call, but I remember much more clearly what broke that anxiety. The first client that the senior attendant and I awoke was using a BiPAP machine to regulate his breathing and keep his airways open while he slept. As the attendant removed the BiPAP mask from the client's face, the client began to sing "My body is a 
cage" -- a song by the Arcade Fire, a Canadian music group. As jarring and poetic an experience as this was, I immediately responded "that's a great song", and we began to talk about Arcade Fire and the music we both liked as we took him to the bathroom in a wheeled "commode" chair to help him shower. That first moment, in many ways, encapsulates the experience that is Attendant Services.

There is a closeness to human vulnerability which becomes almost immediately normalized, but in the very process of normalizing an experience so complex, there are some profound political and social transformations that take place, about which I will speak at length later. The interactions that take place within the framework of the program create a fascinating and strong subculture of disability awareness on these campuses. One may become aware of by reading texts on disability or care work, but the experience of being there and doing it takes one far deeper into an understanding of these things than any amount of reading possibly could.

The anxiety provoked by the idea of caring for another person disappeared with actual engagement with that person as a human being, and as a peer. What makes attendant services at Carleton stand out as a care provider is that the common ground shared by clients and attendants of being university or college students eclipses the differences present in a typical attendant-client relationship. As one client explained, "the atmosphere of your peers helping you was good, I enjoyed that. You get to hang out a bit, you get to make the most of these people. Sometimes it creates kind of fuzzy boundaries, but you felt less like work and more like they were just helping you, which at least for me works out" (I: E : 2013) ${ }^{6}$. There is far more basis for friendship in this regard, but more significantly, this provides a firm basis for social equality between attendants and clients. Another client explained how relationships between attendants and clients are built in the following way: "It's

\footnotetext{
${ }^{6}$ This citation is for an interview. Anytime a citation of (I: [letter], [year]) occurs, this means that it is a citation of an interview. The letter is randomly assigned, and each letter represents a different interview, and the year simply denotes the year that the interview was done.
} 
fifteen minutes every two or three days that you get to talk to someone, and from there if you have more things in common, you start talking about those when they're working, and from there when you're not working” (I: I, 2014).

For the vast majority of the attendants interviewed and encountered in the participant observation, this job is the first contact and experience they have had with disabled people. As a result, there is a subculture which develops quickly and strongly among attendants through their initiation and training as a worker meant to help others that have unfamiliar physical conditions. It is the process of learning to normalize and erase the stigma of disability that is the fountainhead of this subculture. Whether or not attendants realize it (and many indeed do) their experience as an attendant is perhaps one of the most profound political experiences they may have. All attendants interviewed described having their views on disability change in radical ways while working at Attendant Services, and this was especially present in the attendants who had less prior experience around people with disabilities. These very meaningful transformations happened in the context of a mundane environment, attending to the most basic facts of human life.

\subsection{The Structure of Work in the Program}

The allure of being an attendant is, in unromantic terms, that it is a part time job which is flexible in its scheduling, on campus, with management that is sympathetic to students' class schedules and the demands of their coursework, allowing their employees to put their education first. Not all attendants are students, however. There are some who have graduated and continued to work, and some who have left school and still continue to work, as well as people who do not attend university or college. The management are not students, have salaried positions, and have been there much longer. 
There are two team leaders, one each for the male staff and female staff, who coordinate the schedules of the attendants and fill gaps in the schedule themselves when necessary. There is the executive director who has ultimate hiring and firing power, appoints team leaders, writes policy and is in charge of budgeting, securing funding, public relations and an over-arching leadership of the program. The current director has been part of the program ever since it began 25 years ago.

There are two sites administered by the program: the Carleton university residences and the Algonquin College residence. Carleton was the first site and remains the headquarters, with Algonquin college operating as a satellite. In general, Carleton is a busier site which requires more staff (two male attendants and two female attendants during the day) and Algonquin is a small site with fewer clients and staff (one male and one female during the day on staff). Both sites have one person on overnight everyday day. Technically, the different sites are staffed by the same people, but Carleton and Algonquin students tend to work more hours at their respective sites.

At the beginning of their shift, each attendant takes a phone from the attendant whom they relieve, and sign out a set of keys and proxy cards which will give them access to the offices, clients' rooms and storage facilities. During this changeover, the attendant is brought up to speed on what has happened during the day, who has called for help, and given a vague idea as to what to expect in the first portion of their shift.

One attendant carries the "main phone" which receives all incoming calls from clients who need or want help. The attendant on the main phone, in addition to their ordinary duties helping clients, is in charge of dispatching calls to the other attendants. Dispatching calls involves keeping track of where other attendants are and what they are doing, in order to fairly balance everyone's workload, and to give more efficient service by prioritizing more urgent calls over others. Attendants are expected to text the main phone to let that attendant know where they are and what they are doing if a call is taking a long 
time or if they are free to help with another call.

Since the principle of the program is to provide "client-directed care," the role of the attendant is to do more or less what the client tells them to (within reason, obviously). This also means that, since disability is different in every individual the clients need help with different tasks. There are varying degrees of physical impairment, and as such this program lets the clients dictate what they need help with and how they should receive it. ${ }^{7}$

Attendants work independently directly with clients, and in a coordinated team to help meet the needs of all the clients, because there are more clients than attendants. The usual shifts are morning, evening and overnight. The daily tasks are: wake-ups which are scheduled and take place between 7 am and $11 \mathrm{am}$, normally; cafeteria duty, for dinner and for lunch, should the clients want to go to the residence cafeteria and need help with getting and eating food; and bed time, which is not typically scheduled. The overnight staff are, for the most part, in charge of turning clients in their beds who need to be turned every few hours and helping to bed those who choose to stay up later than midnight.

The fact that clients do not have fixed or scheduled bed times does in fact afford a significant degree of flexibility, which is often needed to account for the odd hours that student work and lifestyle engender. As one former client shared, "I enjoyed the flexibility of the care provision, and I felt that it was very necessary in an academic environment when you're not on a particular 9-5 schedule" (I: D, 2013). Many former clients cite this as a degree of freedom lost in the transition from the university program to Independent Living programs in the community.

Other tasks which attendants regularly perform are laundry, cooking, cleaning and washroom

\footnotetext{
${ }^{7}$ Some clients have acquired disabilities as the result of accidents, but most have congenital conditions such as cerebral palsy (CP), muscular dystrophy(MD), and spinal muscular atrophy (SMA). Some of these conditions are degenerative, meaning they get worse over time, but most are relatively stablestate conditions and therefore not "chronic illnesses."
} 
calls. The calls are first-come, first-served, but there is a hierarchy among different kinds of calls based on their degree of urgency. Washroom calls are given the highest priority of all, for good reason - if an attendant is in the middle of helping a client with laundry and they receive a call to help another client with the washroom, the priority is always the latter and thus the laundry will be postponed. Attendants are more or less the common resources of the clients and need to be allocated to the most important and urgent tasks first, as a basic matter of efficiency.

There are some allotted times for things such as going to the cafeteria and doing laundry, but they are flexible within a wide range of hours; a client cannot do laundry during wake-ups or bed times and can only go to the cafeteria between noon and two P.M. for lunch and five and eight P.M. for dinner. Where these policies are not a matter of strict necessity, they are often bent.

When attendants are not doing calls they return to the office which is in the Residence Commons building at Carleton, in an area shared by counselling services. At Algonquin College, the office is in a converted residence room. The team leaders have a separate office at Carleton, and the director has his own office as well, all next to one another. The attendant office is a relatively small, windowless room furnished with a couch, a futon, two desks, a desktop computer, a cabinet full of latex gloves and cleaning supplies, a microwave and a mini-fridge. Sometimes if there are no calls there will be up to four attendants in the office. Clients can come to the office if they need assistance from an attendant office, however, clients are not allowed inside the attendant office. Many clients do come to the office and stay in the doorway to chat or to ask for help, but it is a rather strict policy that is more or less always observed that no clients are to be in the office. It is an arbitrary demarcation of space, but one that could be seen as empowering attendants by allowing them a spot where professional distance can be maintained at times when they are not needed by clients. This division serves the purpose that clients do not always have attendants in their homes when they have no work for them, and so that 
attendants have a place to which they can return to maintain a distance between themselves and the clients. In some cases attendants do spend time with clients even after their work is done and their help is no longer needed.

The space of the attendant office seems to build solidarity between attendants as workers and co-workers. The shared, exclusive space for down-time allows building of community, and to share experiences had on the job, however, attendants not only relate to each other as attendants working together, but also as students who each have their own parallel lives and pursuits (F: 01, 2014) ${ }^{8}$. Comparing this particular program to other attendant care providers in the community, one client explained "there's a lot more division among the attendants because they're all part-time workers, I find they're more loyal to the clients themselves than the task of client care. Whereas a lot of the attendants in the community are loyal to the building”(I: F, 2014).

At the Carleton University and Algonquin College program, the attendants do not share a commitment to the program in the same way as their off-campus counterparts, because the workplace space overlaps with campus residence space and shares the space with the various experiences of campus life. The noise and life of the campus residences also serves to assimilate and normalize the attendant care experience into something which seems to be organically part of campus life, and very unlike an institutional care setting or even another independent living residence.

\subsection{A Gendered Workplace?}

Work is divided by gender: male attendants help male clients and female attendants help females. There are quite a few female staff who work both female and male shifts and will do any male

\footnotetext{
${ }^{8}$ The citation format (F: [month], [year]) is a reference to the fieldnotes taken in the month and year listed in the citation.
} 
calls. Male attendants however, do not cross the gender line in the other direction. Female calls are always taken by females unless it is something completely "non-gender specific." The male clients do not mind being assisted by female attendants, whereas the female clients have a distinct preference to be assisted by females only. The official policy is that no client has the right to request service by someone of the opposite sex. All of this is further complicated by the fact that there have been numerous homosexual clients and attendants, for whom service from, or to, their own "gender" may not be ideal for both parties. Despite this complication, it remains that females do female and sometimes male calls, and males do exclusively male calls. There are several more experienced, senior female attendants who take shifts on the male schedule.

What is perhaps most notable is that besides this formal structure of gender division, gender is not an ostensibly dominant aspect in shaping the dynamics of the workplace. There is no hierarchy between male and female attendants, or between male and female clients. The female attendants, however, have lately been taking more calls because the male schedule has been understaffed, and they can help out with male calls, while the reverse does not occur (F: 01/02, 2014). In this situation where the demand for male attendants exceeds the supply, the females can fill in - male attendants still work just as hard as the females to carry the burden in these situations, but in a circumstance where females need help, there is no possibility for relief from the males and here it may be uneven. Some female clients have expressed openness to being helped by males: "If the male is okay with it, I guess. But I don't know if it was a male helping a female if [management] would allow that" (I: I, 2014).

Some homosexual clients have mentioned that they notice a "queering" of the male attendants with whom they work; meaning that male attendants come to perform their masculine gender roles differently in working with queer clients (F: 02, 2014). This should not necessarily be seen as a "feminizing" of male attendants, but more accurately as a discovery of a different way to express 
masculinity and be masculine. Cis-gender male clients who identify as heterosexual also reported that the experience of disability lead them to explore different ways of both being masculine and of being sexual (F: 02, 2014). Where the role of attendants and clients are not actively gendered in this workplace, there is more room for differing experiences that go beyond binary conceptions of sexuality and gender.

The Program requires its employees and clients to attend a workshop on sexuality and disability soon after they are hired or join the program. Sexual assault and harassment are a concern in the workplace, as most others. Since interaction between male attendants and female clients is, frankly, off the table, the discourse around workplace sexual harassment takes a much different direction. The instances of harassment discussed in this workshop are almost entirely stories of female attendants harassed by male clients (F: 01, 2014). At this workshop, attendants are also informed that they may be called upon by clients to help put a condom on, or to help the client get into bed with a partner. This is rightly touted as an important service that is available to the clients of the program. While in some cases the sexual politics of the program may seem out-of-touch and skewed, it does crucially recognize that people with disabilities are sexual beings, and, without question, deserve access to that aspect of life. But, to paraphrase the words of a respondent, this represents more of a "zero-point" than an actual progressive attitude, and in hailing this baseline as something to be proud of, one gives in to a "zeromentality" which presents things which would otherwise appear neutral as relatively good (I: H, 2014). That is to say, it is better than nothing, provided that nothing is all that one expects. There are still questions around gender and sexuality which the program needs to address, namely that gender is not simply a binary of male/female, and that heterosexuality cannot be assumed as a default.

\subsection{Interlude: The Price of Being Unique}


On a normal day in January, a client and I walk side by side through the heating tunnels that link all the buildings on campus and funnel student traffic in the winter. We walk underneath the pipes and cables on the already low ceilings where the oxygen feels scarce. We both call it "walking" without thinking, though one of us is using an electric wheelchair. As students pass us by, going in the opposite direction, I try to see the look on their faces. I try to read their faces for some mark of prejudice or aversion - a widening of the eyes, a change in the rhythm of their gait. I try to see how they see us. The ones who smile bother me the most, though part of me knows I should not fault them, but I cannot help it. Especially when they smile at me, as though I were doing some great act of charity by simply walking next to a person using a wheelchair, whose proportions are irregular and whose spine is curved more to one side. I do not mind it when they smile at the client, as long as there is nothing patronizing in it.

Some people will regard us strangely in passing if we are simply having a normal conversation between us. And I notice this, I feel their eyes on us, and these ones I do enjoy. I enjoy when we show people, simply by talking, laughing and being comfortably and confidently ourselves, that their own prejudices misled them about what was possible in the world. I know they try to tell if we are faking it, if I am faking it, and I smile more broadly because I see their thoughts written on their faces and feel it in their gaze. I know this because I was once one of those people. I was in a position of where my ignorance and misunderstanding led me to fear people with impairments, or rather simply just not know where to fit it in my understanding of the world. I am, like them, what the client next to me jokingly refers to as "an upright."

He, the client next to me, does not watch the faces and demeanour of the people passing us; a lifetime of being looked at as "that guy in the chair" has made him more or less impervious to feeling 
like he is that guy in the chair. This is what calls my respect and my attention. He knows that where he goes people will look at him, and when he speaks, the very act of him doing so will challenge the ignorance of others. I do not think he is a hero; I simply respect his poise and endurance. Some days he bothers me, other days we argue, but mostly we get along. We share a sense of humour that is darker than most, and we like to make each other laugh.

I used to watch people's reactions much more and react more myself, and I now watch people much less and take more amusement from it. Now I have become like the client next to me: someone who is not halted by the prejudice of others.

I move to hold the doors or otherwise hit the automated door button for him as people step out of the way of his chair, and I follow just behind and beside him. I hit the elevator button and as the door retracts I hold my arm in the way to keep it from folding back out while he gets in. I step inside and push the button for the third floor. We step out into the cafeteria, and I take his student card from his wallet, hand it to the cashier to swipe, and take a meal tray and a couple of straws in my hand. Only our clients use the meal trays, so in a sea of students the attendants can be identified by their keyrings and meal trays.

We wait in the line for pasta, and he is telling me about a paper he is writing on the function of the large intestine is in the digestive system. The line moves slowly and other people are listening to our conversation. Still, when it comes our turn in the line, the pasta chef addresses me instead of him. I step back to allow the client to move forward and with a polite hand gesture I direct the pasta chef to address him. He has trouble seeing over the countertop so I tell him what ingredients are on the line. A few minutes later I take the plate on the tray, and garnish it with red pepper flakes and Parmesan cheese as directed. I ask him if he wants me to get anything else to eat before we sit down, he says he is fine and asks for a glass of milk. 
We go to find a free round table at which to sit, as they make the task of feeding another person much easier. I help him with a glass of milk and take a glass of water for myself. Attendants are not allowed any food in the cafeteria because they are not paying customers. The water gives me something to sip on when I feel hungry as I help my client eat. The cafeteria is full of noise and freshman from residence, and between pauses in our conversation I give him mouthfuls of pasta and sips of milk. My mind skips away from the conversation every now and again briefly to make small calculations as to whether the client might want a drink after so many mouthfuls of food. If I miss the rhythm, he will simply ask me for a drink. I hold glass up to his mouth, the index finger and middle finger directing the straw and holding its entrance above the bottom of the cup.

The phone rings in my pocket. The ringer is perpetually set to the loudest possible volume as a matter of policy and is jarringly loud even in the noisy cafeteria, not to mention on the overnight shift. I answer in the typical policy approved way: "Attendant services, Graham speaking," and I struggle to hear the attendant on the other end above the noise of the cafeteria. They ask me to do a call with another client, I tell them I am already busy in the cafeteria with a client. They ask how long it will be until I finish, and I tell them I have to help the client afterwards in their room and I ask the whereabouts of the other male attendant. They say they will call him and hang up with a polite goodbye.

I turn to the client to ask if he needs help in his room after his meal, and he nods. We go back to his room the same way we came; by way of elevators and underground tunnels. In the wheelchairaccessible residence buildings, the locked doors are triggered to open automatically by our proxy cards and the clients' student cards. We come back to the suite, which has a kitchen, two large bedrooms and a bathroom, all with enough free space to be fully wheelchair accessible.

I get another call from the main phone, and the same call I could not take while I was in the cafeteria has not been attended to yet. I ask again where the other male attendant is, and they tell me he 
is still busy with another client. I tell them "OK, I'll be there in 15." The first client has asked me to help him use the washroom, and the other needs the same. In the bedroom, I grab the sling, put it around the client's shoulders like a cape, and then under each leg just above the knee, with the leg straps looped through one another. He tells me not to forget to undo his seat-belt, in a motion I click it open and tuck it out and under both sides of the sling. I wheel the portable manual pneumatic lift over, drop the hammer on the lift's arm to lower it, and begin to secure the loops of the sling on the hooks of the lift arm. I re-engage the lock on the lift and pump it until my client is hovering half a foot off his chair, sitting in mid-air. I carefully wheel the lift around in a three-point turn, open the bathroom door, hit the lights and wheel him in as he swings gently back and forth. We're debating possible virtues of Disney being given the rights to make new Star Wars movies. "Of course there are many giant cashgrabs to come but at least J.J. Abrams is a much better director than George Lucas." I've had so many conversations with clients dangling from their lifts that the spectacle of someone hovering a meter off the ground, bound up in a sling does not even register as bizarre.

I wheel him over the toilet and disengage the lock on the lift to lower him slowly onto the commode. He tells me "grab the right side of the sling and pull me towards you as you lower" I oblige. He leans back, I unbutton his fly, he immediately leans forward and I help him lower his pants from the back, he then leans backward and I lift one leg at a time off the seat and slide the pants down past his knees.

I stand up and lean with my shoulder to the wall and we continue our conversation as he goes he's spent his entire life going to the bathroom with other people and I've spent the past few years helping people do the same. Neither of us are at all uncomfortable. "Well, I'm done" and I give him a wipe, flush, get his pants back up, set the sling again, attach it to the lift, raise him up, take him out of the washroom, lower him back into his chair, unhook the sling, slide it out from under him and do up 
his seat-belt. I pass him his cellphone and ask if he needs anything else. "I'm good," he says, and I gather my phone and my keyring and at a brisk pace head out of the room, out of the building and across the residence quad to the next client.

He has been waiting forty minutes to go to the washroom. I apologize and tell him we're swamped right now. He says that's ok. I tell him that this is rare, and the program is organized to help everyone with what they need when they need it, and he should expect nothing less. He has just come from another university where their accommodation of his disability consisted of about 15 hours of care per week. He had to schedule all of his needs into about three hours a day every weekday and was largely without service on the weekends except for the morning and the night. Having spent his weekends for the past semester in a state of almost perpetual dehydration, he accepts whatever we give him gratefully. At a recent staff meeting the management tell us if we are having trouble with other clients complaining we don't do enough for them, we should refer them to this new client to hear about his story (F: 01 2014). Thankfully many of us are of the mind that neglect does not warrant further neglect, so we try to do the best for him. This is only his second week, and though he does not think anything about having to wait forty minutes to go to the washroom, I apologize profusely.

I get his commode chair out of the washroom, place it next to his wheelchair, and put the brakes on so it stay in place while I transfer him onto it. I undo his seat-belt, move the foot-plates out of the way and pull him forward so that his feet are planted on the ground and he is leaning against the chair as I hold him upright with my hands under his arms. I pivot and turn with him to place him on the commode. I recall it was mentioned to me that he was close to getting an attendant service program started at his old University. Apparently there were several students pushing for it, with support from members of parliament, and people high up in the university administration onside with starting a program. But suddenly all that ground to a halt, inexplicably. There were whispers that an intervention 
had been staged by some individuals involved with the program at Carleton (F: 01/2014).

Thinking this over, I am sure whatever these people did to stop a program from being established at the other university was done in order to protect the program here, to protect our already paltry funding from being diluted and to preserve what we have managed to do. As I consider how I am justifying this in such a way I begin to feel ill at ease with my own position. Carleton is the only choice for someone with a physical disability if they want some greater degree of independence and freedom when going to an institution of higher education. In that moment I understand that we are not the best, we are the only ones. They come here and the program promises to take care of them, and they do not know any better or have any choice than to believe it. We are unique as a program, but from here it looks like a bitter prize. I hope our hospitals and schools never try to be unique by the same logic.

This has made me uncomfortable. I ask him if he needs anything else, and he asks for a glass of water. I get it for him and then leave preoccupied, walking out into the cold night, twenty below freezing, no coat, back to the windowless office. I check the phone for the time, and become aware of how I am no longer at ease. But it is not so simple to just walk away from helping the people who trust me to be there. My phone rings again, another call to go to. Regardless of the politics, they still need help to get out of bed, and I still need a job to pay rent, and neither of these things will change. 


\section{Chapter 4. POWER AND POLICY: THE SINKING SHIP}

\subsection{Policy and Management in the Attendant-Client relationship}

The dynamic which is usually taken as the defining characteristic of the attendant-client relationship is the dependency of the disabled on the able-bodied. In absolute terms and in the crudest sense this may be true, however it rarely factors in as a definitive characteristic of attendant-client relations, and if it does happen, it is in a situation where a client is being abused by the attendant. An attendant summarized this dynamic rather frankly for me in an interview,

"It's an interesting issue because a lot of the clients say that they feel like they have less power, that the attendants have all the power, but I feel differently. Like I don't feel inferior to them, but I feel that my ass would be on the line if I were to screw up, not the other way around. So I think they're the ones with the power, they're the ones that essentially hold my job in their hands, and if I don't perform well they could easily complain and have me fired for whatever reason. It is client-directed care after all” (I: B, 2013).

The central power dynamic is still one of an employment relation, where the attendant's position is precarious, having very few substantive labour rights as a seasonal part time, student-worker in the health-care sector. The client is the consumer of the attendants' services, and as such has a right to decide which service they do or do not like.

Another attendant added more nuance to this point in saying

"As a friend, I would say they are very dependent on me, and as their attendant, I would say I'm very dependent on them. This job is solely based upon how you can care for them and how you can present yourself without stepping over too many boundaries. And it's really hard to find that line, so I'm definitely dependent on them when I am at work"(I: J, 2014).

If a client does not like an attendant, they may recommend that the attendant be fired, and this is taken 
into consideration by the director who has the final say.

The institutional channels for complaints are in the form of an incident report, or in yearly evaluations. The incident report is written by an attendant or a client when a situation occurs that is out of the ordinary, whether negative or neutral. If a client is dropped by an attendant during a transfer, or an attendant is late getting a client out of bed, or does not respond to a call soon enough given the circumstance, an incidence report could be written. All parties involved in an incident report have to write their own account of the event. The report is sent to the director, who looks it over and decides what manner of disciplinary action should be taken, if any. Staff evaluations are yearly surveys filled out by all attendants and all clients. The rank-and-file attendants have their performance reviewed by other attendants and by clients. Many clients choose not to fill out evaluations, but it is mandatory for the attendants.

The basic fact of the relationship of clients to attendants is that attendants are replaceable and can be gotten rid of immediately if there is a problem. It should be noted that there have been clients expelled from the program due to abusive behaviour towards attendants, but such cases are rare. While this also appears to be the basic fact, it must be qualified in that it can be quite difficult to replace an experienced attendant who is familiar to the clients, and especially one who works a heavy share of hours. There is in fact a fairly high turnover rate of attendants as people graduate, find other part time jobs or more stable and reliable full time work, or simply burn out on the job. One attendant commented that

"When I first started working they would tell me "you're really good right now, please don't become like every other attendant who by their fourth or fifth year they're just shit and they hate working here. Please stay good" And It's crazy that it's like that common of a thing, and they know it, though it doesn't directly affect them, because they're still going to get up in the morning etc. But it still affects them to that level where they're like "please, don't burn out, don't hate this job."'” (I: A, 7: 2014) 
Though the position of attendants in the program is precarious and that of clients is not, the clients are still impacted by that precariousness.

Attendants are precarious labour, but, in an odd twist, precarious labour that is also not easily replaced. One client, having had a problem with one of the attendant, mentioned having certain reservations about complaining: "I had that dilemma again of 'do I get him fired?' Because he's liked by a lot of people and I don't want him to have to go away if there's one client that really prefers him in their care" (I: C, 5, 2014). The clients are reliant on good attendant service for their well-being, and attendants are reliant on clients' satisfaction with their work in order to maintain their livelihood. The emotional and social entanglements involved frequently make these relationships far more complex than a simple employment relationship. Doing one's job well as an attendant largely includes knowing the clients and having established a degree of comfort and trust; even if that trust is broken on the basis of the relationship between one particular attendant and one particular client, the much broader web of relationships those two are involved in within that community may constrain the options open to both of them to exit that relationship.

The clients bear the cost of a high turnover rate, having to establish some degree of trust and familiarity with, as well as train new attendants. This process can be tiring for clients and become impersonal and difficult; sometimes trainees decide they do not want the job even before they are done training - at which point they've already seen clients shower and go to the bathroom. It has been described to me as akin to having a bizarre one night stand, over and over again. One client explained:

"The idea that they are like this twenty or more people, it's like this revolving door where every single day there's this new person who is there that sees me incredibly vulnerable. And the fact that this happens every single day, on a subconscious level it becomes overwhelming to have all these people. Particularly, I kind of need to psychologically prepare myself when there are new people that are being trained. I find it incredibly invasive sometimes, and usually it's without notice too. Sometimes I just wake up in the morning and I see a stranger, and they just see everything. Just when you're comfortable with 3 or 4 people someone else comes in" (I: H, 
2014).

The training process has some drawbacks for the clients. There is sometimes not full disclosure to the attendant trainees of what the job entails, and the trainee only really finds out on their first day. It can make an already stressful and awkward situation worse when the trainee does not know what they are walking into, and the client sometimes is not told if someone new will be walking in to their home in the morning to help them out of bed and take a shower.

\section{2 "Telling a Dog not to Bark" : The Short Arm of Policy}

The attendant Code of Conduct provides the fundamental rules for attendants to follow in their interactions with clients and as employees in the Program. All attendants are required to read it and be tested on it as part of their training. It is composed of the following ethical points:

1. Attendants shall treat clients as equals when providing service.

2. Attendants shall deliver service in a respectful, courteous and timely manner. Attendants will provide service in a manner so the client is not unnecessarily placed in an embarrassing situation. The attendant will use discretion where appropriate.

3. Attendants shall respect the rules of the Department of Housing and lor Campus Living, including quiet hours. Attendants shall also abide by all regulations set out by other school departments and services. Attendants shall not assist clients to contravene any school policy.

4. The Program shall not deal with social interaction between clients and off duty Attendants. However if personal conflicts cause a situation where a Client and Attendant are unable to work together, the consequences of this situation shall be borne by the Attendant.

5. Attendants shall not work for Clients outside the program unless approval is given by the Executive Director.

6. Attendants shall not come to work under the influence of alcohol or other drugs, which may impact upon their ability to prove service.

7. Attendants shell not assist any Client to perform illegal acts. 
8. Attendants shall not lend or borrow money to/from Clients.

9. Attendants shall not complain to others about Clients or other staff of the Program outside of the proper Complaints Procedure.

10. Attendants shall not break rules of confidentiality.

11. In order to provide effective and responsible service to the clients, attendants will learn and regularly review the Polices and Procedures of the program.

12. Attendants are obliged to report any breaches of the Code of Conduct either by themselves or by other Attendants. (downloaded January 8, 2014, from attendant services forum)

Thus far in this section I have given a face-value appraisal of these and other rules of the program, but in order to understand how the complex social order of Attendant Services actually works, we must consider not just how these rules are followed, but which rules are systematically bent or broken and how, as well as why, these transgressions are treated as acceptable. In addition to these explicit rules, there is a large network of informal rules and practices that address ethical issues where a more context-dependent framework for action is needed.

Many of the formal code of conduct rules seem fairly obvious and commonsensical, but the most important are 1, 2, 4, and 10. Hidden meanings abound in these particular rules which are not apparent to an outsider. Perhaps attendants believe that it goes without saying that we must treat clients as equals, but this is hugely important, important enough that they are included in the Code for a number of reasons. First of all, it provides a political underpinning for all interaction under the umbrella of the program; not only are clients to be treated as equals to their able-bodied peers, but attendants themselves, in treating clients as equals, also establish the grounds for the attendants to protect themselves as workers - to not be treated as "servants."

Rule 2 stipulates that attendants be generally competent in carrying out their duties which, again, might appear a statement of the obvious. But the importance of both being able to use discretion and 
explicitly trying to avoid putting clients into embarrassing situations are extremely important not just as actions, but as ethical principles. This, again, is a broader ethical principle that is necessary to avoid abuse. Most clients rank social equality as one of the most important aspect of their relationship with attendants (F: 01, 2014)(I: 2013-2014).

Rule 4 addresses the issue of friendship and places it within the power dynamics of the attendant-client relationship. This rule explicitly states that the attendant is the one who, in the eyes of the program, is replaceable and thus bears professional responsibility for a friendship gone awry. This raises a problem in light of the testimony of every attendant interviewed who, one way or another, all spoke of having naturally established friendships with the clients, and saw the dominant aspect of the job to be social rather than physical. As one attendant described in an interview,

"I think it's the social aspect, for sure [that is the greater part of the job]; going in there, being friendly, pleasant, and making their day better. That's the big thing, that is the job, for me. Feeding them is such a small part of the job, you have to walk there, you have to show up to the room, you ask them how they're doing. You spend way more time talking to them than you do help assisting them." (I: K, 2014)

The tacit meaning of this rule then, after one understands what the job of the attendant entails, is that it is the attendant's job to maintain an amicable relationship, and if the clients do not like them, their employment could be at risk.

One of the more junior attendants interviewed understood their role as an attendant as "I'm their friend, that's it. I'm their friend that's there to help them, and I'm paid very little to do so"(I: K, 2014) Another more senior attendant testified that "The manuals that we read when we first get hired are so outdated compared to what we tell people when we are training them. To say to not be friends with the clients is like telling a dog not to bark" (I: J, 2014).

All evidence found in this study points towards the fact that attendants in this program, though 
advised by the rules to be wary of friendship with the clients and become friends at their own risk, almost inevitably develop friendships with the clients. Furthermore, in the experience of the attendants interviewed, the social aspect of the job was seen to be the most dominant with physical care being almost secondary. Doing well in the job depends more heavily on social interaction than on the technical aspects of doing care. In the words of one interviewee,

"When I was told that there were evaluations, where people would judge you based on your performance, then I realized it wasn't how well I could give care, but how well I could communicate with clients to make them actually like me. Because the care part was easy, it was getting them to like you for you instead of just for their care. It's very social instead of just personal care. If you're good with the care, then you're good with the care, but if you're good with the social aspect as well that's the way you'll get noticed and get good evals.”(I: J, 2014)

This characterization may seem to point in the direction that attendants are at the mercy of the clients, but this would be an over-simplification. It is not a perfect system wherein all clients always actively put good care or even amicable relationships ahead of the interests of attendants.

The feedback and report mechanisms available do not create a self-regulating market for the best attendants, because often clients choose not to report attendants for bad behaviour, and also not to evaluate them. Often it falls to the other attendants to police and regulate their colleagues, though their ability to do so is limited because they do not work directly with one another. Working in parallel means that attendants do not witness the larger part of the relationships between other attendants and the clients, and therefore the attendants have less recourse to report transgressions by their colleagues unless the clients do so in the first place. One attendant described it thus:

"If any of the clients have problems with any of the other staff, they don't IR things, and we get in trouble when we start bitching about it. There have been many times when a client has told me "I don't like this person, they messed up this, this and this," and I'll have to go tell [the team leaders]. And they'll be like "did they IR it?" And I say no they didn't because the separation between client confidentiality is so bad, like everyone knows everyone's business. The first thing management say is "this person is talking about you, what did you do wrong?" And then there are the evals where for "protects client confidentiality" everyone puts a [high grade], because nobody wants to out another person because then we'd all be outed." (I: J, 2014) 
Regulation of attendant behaviour is also very closely related to the culture of confidentiality (or rather, lack of confidentiality) in which the management, the attendants and clients are all implicated. Issues of confidentiality are addressed in Rule 10 .

Rule 10, concerning adherence to confidentiality, is perhaps most interesting for how it is interpreted and observed within the workplace. Confidentiality outside the workplace is more or less adequately observed, however, within the workplace itself, this is a far more slippery issue. In running a program, providing adequate service and governing clients and attendants, there must be a degree of talk about issues in the workplace, including information about clients and other attendants. Attendants, in discussing their work are ultimately discussing the clients, and clients, who discuss their care with their attendants, are usually discussing the care of other attendants where it has perhaps been lacking. These discussions operate as a kind of collective self-regulation within the program, but is by no means unproblematic or frictionless.

It may be very difficult and even undesirable to too strictly observe confidentiality among attendants. In talking about clients, attendants learn how to better serve their needs or interact with them. One attendant interviewed described this as "shop talk." This talk should not be malicious, and is almost never malicious, but can sometimes objectify the clients. The fact is that the forum which attendants use for scheduling, discussing and receiving new policy, or keeping up to date on routines of clients and important notes on their care, does share sensitive information about the clients broadly with all attendants.

Confidentiality does exist between the workplace and the outside world, but within the workplace seemingly "personal" or "sensitive" topics are discussed, almost as a matter of housekeeping. Indeed, one staff meeting was turned into a kind of workshop on what to do with policy in 
regards to problems that were occurring with specific clients. The issues raised were of the most sensitive nature, and were furthermore shared openly, by management and with the entire cast of attendants, regardless of whether they had ever served or would serve these clients.

What could properly be called "gossip" in the program is not often personal care-related, and this distinction is worth making. One client noted "I don't often hear about other people's care, like how they go to the bathroom, but in terms of issues not related to personal care I would say that there is a big problem with confidentiality at attendant services" (I: I, 2014). The fact that the tenth rule attendants must not break confidentiality -- is consistent only in how inconsistently it is observed by attendants perhaps affords them a more subtle means to exercising power over clients. The power to waive confidentiality is not a power of the attendant only, but rather an oppressive structural power within the Program. It can be toxic to the workplace because it both weakens the position of the clients and degrades trust of the attendants and management in general.

Many clients found that poor or selective observation of confidentiality was a very problematic aspect of the Attendant Services Program, and some actively tried to distance themselves from the program because of this. One client shared:

"I had a relationship with one of the other clients in the program... and I felt like when that was going on, nothing was really safe between me and the person I was seeing, and there was always someone else who had an input. There were always attendants involved, and they were very particular people, but they were good at their job. I didn't really feel comfortable complaining too much because it would be to the detriment of those clients who actually functioned really well because of these other people. So you have to manage these personal relationships with these people, tell them to back off or whatever, even though you really didn't sign up for it." (I: F, 2014)

Some respondents perceived Attendant Services as a perverse and somewhat suffocating subculture which needed to be kept at arm's length. This sentiment also made it quite difficult for the clients concerned to get the help and support they needed, because they felt if they did, they would be subtly 
pressed into consenting to an invasion of the private aspects of both their inner lives and their social lives.

"There were some major privacy issues. ... I found that attendant services within Carleton created this kind of really unhealthy clique, where the affairs of the clients were shared between anyone who had more than a professional relationship with the clients. If there were people who had a less than exemplary character they would, you know, very much spread that dynamic. (I: C, 2014)

This perspective was held by clients who required more help in their daily activities as well as those who needed less and were more functionally independent - feeling surveilled and limited by the program was not dependent upon having a certain level of engagement with the program.

Attendants reported having parallel issues with confidentiality. As an attendant explained in an interview, 'It's also not like a job in 'the real world' where you can take your grievances up the next rung in the hierarchy; confidentiality is not respected here at all” (I: G, 2014). Attendants found it difficult to deal with problems in the workplace that were of a sensitive or personal nature without it being known to everyone. This was an area where both clients and attendants alike insisted that policy needed to be more strictly enforced, and that management did not do their job to enforce it.

In contrast, clients and staff complained that policy was too rigidly enforced in areas where policy and procedure were perceived as backwards or outdated. These issues emerged in cases where policy could not be enforced upon everyone because it impeded the ability to meet the needs of some clients. An attendant respondent explained:

"What happens if you have a client who is bed-ridden who severely needs the things that are coming in the mail from the desk? We are not allowed to borrow cards, we are not allowed to pay for things, and if you need to pay for the package that was delivered at the front desk, you have to take their card, you have to sign off for them, you have to hope that the front desk people will believe you that you're an attendant and you have to use the clients money to pay for the thing. All of those things are against our rules. But yet the person who is in the bed really needs that stuff, so you have to bend and break the rules where you see fit. So professionalism in this program is really hard to define, because you're leaving it up to who is defining. Maybe the client thinks that you are being completely professional and completely confidential by not 
telling your bosses about that breach of the code. It's not a one size-fits-all program.” (I: G, 2014).

Attendants and clients alike often push back against policy that does not fit their needs, and do so because they have little faith in the management's willingness to respect their concerns. These circumstances affect the dynamics of the work culture, as is addressed in the following section.

\subsection{Together on a Sinking Ship: A Culture of Resistance Against Management}

One of the first things that became apparent about the workplace culture was a strong distrust of the management by both attendants and clients. This is perhaps nothing unique for a workplace, but it is nonetheless significant that in the majority of the opinions on management recorded in the fieldnotes, virtually all of them expressed that management was at best an incompetent governing body with good intentions, if not simply seen as a hindrance with a backward agenda. One respondent summarized this general feeling quite well: “They've been there since the dawn of time, and they've nestled themselves into this little environment that caters to them, but they have not really changed with the times. That's why you have somebody who is running the program who never goes on the floor, and never really attends himself to the issues that are being faced by the clients at a broader level" (I: C, 2014). The management is commonly viewed as absent and out-of-touch.

One attendant reported a situation when "The director asked [an attendant] what the name of [another attendant] was the other day... [that attendant] has been here for years! When was the last time [the director's] been on the floor helping a client? He doesn't know what they need, or what they want, or how they do their care or if we need any innovation" (I: G, 2014).

At the team-leader level, that is of the on-the-floor leaders of the attendants, who have salaries 
and benefits and are in charge of scheduling, the common view among attendants and clients is that they are disorganized and unreliable. An attendant shared that "I've had numerous clients tell me: "don't let [the team leader] come and do my wake-up, please do it instead, I don't want [the team leader] to like harass me while I'm getting woken up." And I'm like, how can you follow that example?" (I: G, 2014). Another attendant relayed that "A client threatened to go to the director after, for the entire summer, the team leader had been showing up to his wake-up 45 minutes late" (I: K 2014). The disconnected senior management, along with the disorganized junior management together create less than ideal working conditions for the attendants and less than ideal living conditions for the clients.

There is an understanding among the attendants and the clients that they are more or less on their own in keeping the program functional (F: 02, 2014). The management does not provide particularly strong leadership to the rank-and-file staff, or inspire confidence among its clients. The relationship between attendants and clients takes a more central role when the management is not seen as fulfilling its role. A senior attendant shared:

"I feel like we're all stuck on a sinking ship together. I was talking to a client and she described it like "I'm really glad I'm getting off this sinking ship before it sinks completely." Because a lot of us in the program, and I use it collectively from what I've heard from attendants and clients, have the perception that this program is going downhill fast. There's nothing that's going to stop it. I know that a lot of the better attendants are going to be leaving, and at the same time there's going to be nobody who has been around for a long time who understands the dynamics of the program to keep it from completely collapsing. I feel like a lot of the better attendants fill in the gaps that the lack of innovation in this program leaves." (I: G, 2014).

Consequently, this puts more pressure on the work relationship of attendants and clients because both parties feel the need to take more responsibility into their own hands.

One client explained "I hear stories about management and their treatment of attendants, and that really frustrates me, especially if they are friends with me. Because it's just like someone is being mean to your friend" (I: I, 2014). This is indicative of the kind of relationship that is forged between 
attendants and clients in such a situation. In a similar vein, an attendant expressed:

"I'm glad I only have one more year, but it's so hard to quit a job when you make such good friends with these people. That's the only reason why I'm here. The fact that we're not getting paid for a month kind of pisses me off, but the fact that my friends are here makes it difficult to leave. [A client who is also my friend] keeps telling me to quit. She says "you do not deserve to be here," she says "quit." But then I see who they're left with and there's no way I can quit." (I: J, 2014).

What was shared by this attendant perfectly demonstrates what happens when the responsibility is shifted from the management and policy to the attendants and clients themselves. Both attendants and clients view the attendants as the anchor of the program, and further, that this is not a desirable position.

An attendant explained that

"It stresses a lot of [the clients] out. I definitely hear a lot of complaints about our job, not what we're doing but the conditions we're doing it in, from [the clients]. ... A lot of the clients are like "thanks so much for helping me, do you want a snack, this and that, this and that... [they say] "it's shitty you're scheduled this way," or "you really need to be paid more" or "I wish I could run the office and make this better for everyone."”(I: A, 2013).

The attendant went on to say

"it's that stuff that makes me a grump at work, it's a bad workplace atmosphere and when you go into someone's room, they feel bad asking for anything. I'll try to hide it and put a smile on but they know. And they just know in general that the conditions are so shitty and they feel bad asking, and they feel bad asking for extra help. ... I feel like it affects their experience too, and makes them more aware that they're disabled, because it makes them feel extra bad asking for anything. I know we have some clients that feel so bad, and they're apologizing constantly when they're asking for anything... but that's when they come to us in first year and eventually they'll get more comfortable asking. But then it also swings back and they start to realize how shitty we have it and feel really bad." (I: A, 2013)

The working conditions of the attendants directly affect the living conditions of the clients, because the workplace is literally their homes, and the work of the attendants is helping with clients' basic needs so the presence of the Program is always felt.

Clients are often placed in a position of gratitude which, as the attendant quoted above mentioned, makes them feel further disabled because they feel guilty when asking for things they 
simply need. Another attendant shared:

[One of the clients] has admitted that she's begged some people to stay, because it's just one more person you can rely on for quality care. Which is sad, because it's a guilt relationship that is being brought in now. I've had other job offers, and the private clients that I work for are obviously paying better than this job now. We get severely underpaid and over-worked, and this job just brings so much stress, and the cost-benefit is not equalling. (I: A, 2013)

Yet attendants stay for the clients whom they serve, and the clients try to get the attendants whom they trust to stay because neither see any better option open to them.

The hallmark of this workplace culture is that loyalty is not typically given to the program, management, or policy but is held between the attendants and clients.

"[My loyalty is to] the clients, for sure. [Another attendant] and I talked about it, and we would not be here if it were not for certain clients who we felt an obligation to. How would you feel leaving your friends on a sinking ship and taking off? Who's going to come and pick up where you left off? I started school alongside some of these clients, and I want to see it through until they graduate. They'll graduate and I'll graduate and we'll be done with this together." (I: G, 2014).

There is solidarity between attendants due to the conditions that they share, and this solidarity translates into a culture of resistance against governance by management and policy. This culture is the result of dealing with a shared experience of precariousness. 


\section{Chapter 5. THE MUTUAL EXPERIENCE OF DISABLEMENT}

\subsection{Neoliberal Training-Wheels or Emancipation? :}

\section{The Transition to Independent Living}

Within this shared space of the university, the attendant-client relationship also takes on a particular character, and that is one which virtually all the respondents described as a unique peer-topeer kind of relationship. The basis for friendship was seen by respondents to be much more present than in other independent- or assisted-living residences out in the community. The shared space and identity as students makes the divide between attendants and clients into less of a defining dynamic in the relationships that develop at Attendant Services. This also serves to make the attendants more loyal to the clients than to the program itself. Shared experiences, though, lay a particularly strong foundation for not just friendship, but social equality between attendants and clients. As one respondent noted,

"I think that has a lot to do with age, what's great about Carleton is that we're roughly the same age. When you think about it, why there's so much sexual politics and other things at Carleton, that is the time of your life that you work these things out whether you are an attendant or not or a client or not, so to have people your same age going through the same things because you're all working on assignments and courses." (I: F, 2014)

This dynamic helped to create less of a binary divide between attendants and clients and allowed a 
different kind of interpersonal space to emerge where care was less work-oriented and more social.

"At Carleton I was definitely a lot closer [to the attendants], it was a lot more informal so we would hang out during and outside of when they were on shift. And because of the fact they were students, we definitely had a lot more in common. Not just age, but we're both studying at Carleton, and we had similar interests, and age obviously plays a factor, a common environment - a lot more in common. It was a lot easier, at least for me, to get closer to those attendants. Which as I said, is good in a lot of ways, because for me it made it a lot less uncomfortable when you have a rapport with the person who was helping you in those situations.. But also it was more fun. Now I've developed friendships where I go out somewhere and I can count on people who come with me to understand that I need help with a drink and there's no confusion with that. It adds less stress because of that whole friendship thing." (I: E, 2013)

The context-grounded relationship of student-to-student, rather than solely attendant-client relationship offers considerable space to allow for a different kind of engagement with and experience of disability, for both parties.

When asked about their relationship to the clients in an interview, an attendant expressed that "I wouldn't really see them any differently than I would my friends who are able-bodied" (I: B, 2013). Among the more senior staff in particular this attitude dominates. Another attendant said "I've told them "I don't see you as someone in a chair." When I got out with some of them--some of them are my really close friends-- I see them as people who are just my friends and I don't picture them in a chair because that's not what defines them"(I: J, 2014).

For newer attendants the change in attitude is quite clear, one respondent told me "I've definitely become more comfortable, I feel more comfortable facing them like I'd talk to any normal person. It's increased my comfort level touching them, talking to them, joking with them. And it's not that I wouldn't do that before, but I never got the chance to. I would have been fearful like.. .asking them what they have unless I knew them" (I: K, 2014). The honesty and open naïveté of this quote may seem problematic to those with a refined sense of the semantics surrounding disability, or the fact that someone even needed to learn these things may in itself appear offensive. But once again this is an 
honest expression of one of the elementary struggles of disability culture, which is to replace ignorance and fear with understanding and humanity. While the horizons of this culture and this struggle are always expanding, it is in the lived experiences of people both with and without disabilities that these struggles actually take place.

None of this is lost on the clients of the program. As one client said about the process of establishing a good working relationship, "for me it has to be reciprocal. And I think in the past I never realized that not only is the attendant offering me something, but I in turn offer them something too. I think this is the kind of experience that changes both parties. It really does. It changes me in a really profound way and from what I've been told it changes the person that provides the care: the attendant too" (I: H, 2014). Change and transition are recurring themes in the program.

The Program sees itself quite explicitly as facilitating the transition from home care to the much more formalized and professionalized mode of independent living residential care in the broader community. As one client who had recently made the transition explained candidly:

"Well I've heard them say this over and over again, that they view themselves as sort of a transition, and that was one of my anxieties about the thing. Like, in their mind, we move from our parents' homes where we've been supposedly coddled all of our lives - the traditional narrative-- and then we move to there as the place where we get our neoliberal training wheels and we prepare for the life society has dictated we have because of economic conditions. So I think they view themselves as preparing the way for us to be productive members of society. [The director] has engineered it in such a way that his favourite clients or clients that he sees show the most promise get positions out in the community quickly so that we can lead functional lives. I think the program views itself as a transition thing from parentalism to state funded social programming. (I: F, 2014).

The notion that the program is a transitional stage for its clients suggests a particular manner of socializing people with disabilities into the "mainstream" of society.

The question is, then, what kind of "disabled subject" arises out of this programme? It speaks of a disabled subject who knows what to expect in their lives. This is a crucial process of learning both 
what to ask for, and what not to ask for from others. The program teaches the clients about how to follow the rules, but more importantly about how the rules can and will be acceptably broken by themselves and their support workers.

The next logical question is: how is the role of the attendant instrumental in this transition? One client described his experience interacting with attendants:

"At Carleton, as problematic as I think this is to a liberal care-based model, it was refreshing to have some people say "no, I'm not doing that" or "ok I'll do that but I really don't want to" and you have to negotiate with them or explain why you want it done. It's much more about independence through consensus" (I: F, 2014).

This kind of social aspect is embedded in the everyday work of the attendant and is integral to achieving the broader goal of "transition" around which the program orients itself.

The ideal attendant for clients who have gone through the program is not necessarily the friend, but the trusted figure in the background with whom friendship is possible, but not necessary. One client explained:

"I eventually got it so that I had people follow me around sort of receding into the background as I lived my life... [so that they were] non-intrusive" (I: F, 2014). Similarly, another client expressed that "there are certain attendants I have a really wonderful relationship with, and wonderful not as in, you know, I wouldn't necessarily call them friends or hang out with them on the weekend, but wonderful in that they fit seamlessly into my life" (I: H, 2014).

The transition of the clients of the program is in learning the informal rules of how to behave with attendants, and how to set up that relationship in order to facilitate their own functional independence.

"I think first year I wanted to be everyone's friend. First year I was grateful for every little thing that they did for me. I was incredibly polite, I'd like to still think that I'm polite and that I'm not a bastard when I'm speaking to people. Certainly first year I was somewhat shy but you know, operating from this place of this perpetual gratitude. And now that's started to change. It's not that I'm not grateful, but now I begin to see it as, I don't know if this is good or bad, but I begin to see it more as you know, transactional. ... I'm starting to embrace the messiness of it, but also that I don't have an obligation to feel grateful. That sense of gratitude is not just a disservice to 
me, it's a disservice to the other person as well, and I'm only starting to realize that now." (I: H, 2014).

Clients are taught how to be effective managers of attendants, and how to main the balance between work and friendship that is required in a healthy care relationship. The transition is much deeper than learning a skill set, it also deals fundamentally with subject formation. With the client quoted above, explaining that gratitude is a disservice to themselves and their attendants, there is a recognition of themselves as a certain kind of subject. There is the recognition that they are not recipients of charity, but a social equal who is receiving what they deserve.

While client-directed care does imply that clients do work in directing care, it still leaves the actual task of care to another. There is, however, a great deal of work done by the client themselves to secure effective service. Interestingly, this client phrased their role in care in much the same way as many of the attendants did:

"I think it's a tough balancing act because you do need to retain professionalism when directing, so that you're ensured that you're getting the proper quality of care. So in general I think that I did a good job of making that distinction between instances where I felt I had to be professional and where I could be more social and friend-oriented. And in general, I was friends with all of the attendants and I got along with them well. And the attendants themselves had a good notion of when to separate a friendly relationship from a working relationship." (I: D, 2013).

Client-directed care also implies that clients take responsibility for themselves and for their care, and actively manage that relationship in much the same way the attendant does.

\subsection{Why Do We Care? The Attendant Experience of Disablement}

Although attendants are meant to facilitate eating and bathing on a regular basis, it is beyond their authority to insist on particulars of hygiene or diet. This is in line with the client-directed care model, and is primarily to give clients the most choice and control over their lives, and in turn get rid of 
paternalistic attitudes among the attendants. But in a situation where one person depends on another to do things essential to their well-being, the agency of the helping person can never be fully erased. It is a sensitive political boundary, but it is by no means a boundary that can ever be absolute; often clients rely on attendants' suggestions of how to do certain routines better or more efficiently, and sometimes simply on personal advice. The clients are the experts on their needs and bodies, but attendants, as much as they are theoretically supposed to be surrogate arms and legs, often become experts on how to best carry out the process of meeting those needs.

The word "care" comes up a lot in the workplace as a matter of habitual and common use, but those concerned with political semantics would most likely characterize what attendants do as help rather than care. The difference being that "help" is something that takes place between peers and relative equals, whereas "care" has a paternalistic and institutional attachment and history. Nonetheless, most attendants do talk about their work as "care" and most clients also talk about the service they receive as "care." Indeed, though the attendants care for the clients, it is frankly difficult for most attendants to not also care about what they do, because a condition of doing the job well is understanding that the actions of the attendant have a direct effect on the quality of life of the client who receives the care. Clients take an active role in receiving their care through directing it and providing feedback and engagement. Care does not imply paternalism in this case, but a mutually executed process.

Balancing "professional detachment" and caring is perhaps the most draining part of the job. It is also important to note that the two do not exist on a sliding scale where some attendants are more detached and care less on one side, and other attendants care more and are less professional on the other; these are two contradictory aspects which attendants deal with simultaneously, and it is not uncommon that people who "care" the least will also be the least professional, and those who care the 
most will more likely be the most professional. One client explained that "when you're friends it just makes it easier, and when they're not being professional it stands out more" (I: I, 2014). Attendants tend to be held to higher professional standards rather than lower standards by clients whom have become their friends. Trust is the currency in the attendant-client relationship, and with more trust comes a better standard of care. Professionalism and care can be mutually reinforcing, but the fact is that it takes considerable and constant effort to achieve this balance.

While the role of the attendant is clearly outlined and the policy appears to be very straightforward, it is important to remember that human interaction is a very complex thing and in the face of real life, policies often lend themselves to unexpected interpretations. An attendant noted that "We walk this line between professionalism and friendliness, and we just kind of sway between because it is dependent on your relationship with each client" (I: G, 2014). That is to say, the work of the attendant is difficult to understand without actually being one and having a multitude of different relationships in that capacity. The kind of labour the attendant does is unique because it is not only alienated in the sense that it is sold on the labour market for a wage, but also in that the "product" of the labour is the intimate care of another human being. It can be both reduced to the aspect of money, or alternatively cast as an paternalistic kind of altruism, but neither of these explanations have stood the test of research. It became apparent that the worker is squeezed from both sides: they may not be paid enough to care; or they may be convinced they care too much to be paid (well).

There was not a single attendant respondent who did not find the job to be emotionally draining. The basic fact is that "If you go to a client, and they're having a shitty day, and they're stressed out and they're crying, it's going to rub off on you and you're going to feel more stressed out" (I: A, 2013). When asked if they found the job emotionally draining, another attendant responded "Yes. Especially for long shifts; I go home and I am just happy to be by myself in my house. I actually turned down 
living with a friend this semester because I felt like at the end of my day all I want to do is go home and be by myself... and I'm a very social person"(I: G, 2014). Attendants also blamed their stress on the management, saying "There's the stress that our bosses put on us all the time, and if it were not for the people, I would not be here"(I: G, 2014). This was a common sentiment.

On top of problems arising out of frustration with management, the level of stress is further exacerbated by the dynamic of friendship which can explode easy answers on the appropriate role of the attendant. One attendant explained:

"When you read the manual ... it basically tells you not to be friends with the clients. What does that mean? -- the only thing I find that's really hard with our job is that we're not only attendants, we become psychologists, we become therapists etc. ... Where do you draw the line and say "I can't help you with what's going on in your life" I think that's the hardest part to draw a line on." (I: J, 2014).

Caring for and about clients and maintaining professional detachment in interactions with clients are two aspects that are often in direct tension with one another. Policy demands detachment while human beings demand connection. While some attendants and clients do remain "professionally detached" in their relationships, most do not, and most attendants do not seem to derive much satisfaction from their detached interactions, and this is indeed also the case of clients. Detached service is adequate, and adequate service is the baseline for fulfilling the clients' right to support, but actual positive interaction is a much more powerful and important experience. It is unlikely and unrealistic to make "having positive interaction" a policy but this dynamic of practice shows that policy can be only a framework to create a space where these care relations become possible.

Professional detachment, as it is actively deployed by the attendants in this study, did not mean a cold or distant demeanour but a degree of role-playing. What I mean by role-playing is that attendants have to, at some point, recognize their position in relation to the clients as workers who are paid a wage to do a job, rather than simply being their friend or their saviour (and/or stooge) by doing whatever the 
client requests of them.

As described by many of the respondents and participants, balancing friendship and the role of attendant/client is the most difficult but also most rewarding aspect of the job. When asked about how they saw their relationship to the clients, one attendant said "for the most part whether I wanted to or not, they've just really just become my friends"(I: B, 2013). Furthermore, this testimony is representative of the attitudes that the majority of attendants took towards the clients. Professional detachment in this case requires that the attendant still occupy the role of attendant first and foremost, before being a friend. So what detachment in reality demands is a kind of social negotiation between attendant and client where both play their respective roles and not let friendship skew the terms of that relationship in one direction or the other.

Professional detachment as a requirement of professionalism should be here reformulated as "professional engagement" to better encapsulate the fact that care requires engagement. Where friendship exists between an attendant and client, they are usually more likely to have a better working relationship because it helps them to more easily come to a tacit agreement on where the line between the duties of work and the expectations of friendship should be drawn. Erecting and maintaining this boundary is the work of both the attendant and the client, and is instrumental to building a trusting relationship. This is an active engagement, not a product of detachment. There are, however, wrong kinds of engagement.

There are certain situations in the overlap between friend and work relationships wherein the client may be pressed upon by the attendant to let them cut corners in their care or extend a break time. This was, however, in the feeling of many clients, not a common occurrence or a significant problem. "For some, very few attendants, the friendship aspect could lead to them being lazier with your care and affect the standard of care. And also it could lead - this didn't happen to me personally all that 
much - but it could lead to certain situations where attendants took advantage of the friendly relationship they had with clients" (I: D, 2013). Another client when asked if the friendship aspect created unequal power relations for them said "there were like maybe a small group of one or two people who I could think of were really close friends so they would try to encourage me to sleep in or that I didn't need a shower that day. I think that was because they thought our friendship would work in their favour in that way. Generally for the most part, definitely not" (I: E, 2013).

The opposite situation, of the attendant-friend being persuaded to work above and beyond the scope of their normal duties by a client, was by far a more common experience of the attendants, as well as a defining problematic of the work itself. One attendant who described the clients as friends explained "some people would never ask me for things but some people... like a big one is asking me for help with like things that they know that I shouldn't be helping them with. Things like alcohol and stuff like that, that I have to say 'sorry I know we're friends but it conflicts with what I'm able to do for you as an attendant'"'(I: B, 2013). It has a great impact on how the attendant is able to maintain professional distance while they are expected to be present both as an attendant and as a friend. Though the technical aspects of the job are foregrounded, and understood to be relatively straightforward, it is this balancing act which is the most difficult and essential thing to learn in order to succeed as an attendant.

A common experience of attendants starting out on the job is that they, in their newly acquired role, do not yet comprehend the complexity of their relationship to the clients. For many new attendants, for whom this job may be their first encounter with people with disabilities, they may be unfamiliar with how to interact or even at times uncomfortable with the intimacy of the things they are asked to do for relative strangers. They are often very eager to please, and thus can be readily exploited, because they do not yet fully understand their own position as workers (F: 01, 2014). Socializing a new 
attendant into the workplace culture takes time, and during that time they may not apprehend their role fully. What some people seem to anticipate is that they will be saviours in their capacity as workers. They want to be friends to the clients, and they want to be genuine in helping them. All of this is very noble in intent, but comes from a place where disability is still a foreigner (F: 01, 2014).

These attendants do not yet know how to say no to a client's demands when they are unreasonable, that is, either outlandish or of a low-priority as far as attendant calls are concerned (F: 12, 01, 02: 2014). For the new attendant, the novelty of interacting with a person with a disability may have been normalized after the first day, but in order to be a mature attendant one must normalize disability itself. In saying some attendants fail to normalize disability itself, it is meant that some still hold a misguided notion of their moral duty to help people who they have discovered are just like them, but are at a disadvantage because of impairment as well as the concomitant oppression they may face because of it. To normalize disability within the attendant-client relationship is to understand that disadvantage, but also to give consideration to one's own position as a worker who shares in that situation. One newer attendant explained how they perceived their role:

"I'm their friend, that's it. I'm their friend that's there to help them, and I'm paid very little to do so. I'm paid so little that I'm not doing anything extra, everything I do at that job I do because I want to. I'm helping them, and I'm not going to do a shitty job because these are real people. I do a good job because I respect them as people, I do a good job because I have to, I want to: not because I'm told to. ... I do a good job because I want to treat these people well and they deserve to be treated well, that's the only reason I do a good job. And because I like these people, they're my friends, I mean I look forward to coming into work to see what they're up to. I like helping them, I'm their friend." (I: K, 2014).

Once again, this is not an uncommon or improper reaction for new attendants to feel so strongly this way, but eschewing the monetary aspect wrongly assumes a foundation for the relationship which has not been consented to by both parties. A client explained in an interview that "At Carleton.... I think there were times I was expected to be a friend when I just wanted a work relationship type thing” (I: E, 
2013).

There is wide agreement that a certain distance between attendants and clients that must be maintained to make sure neither the attendant or the client is being unnecessarily imposed upon by the other. An attendant noted "I find the ones I get along the best with are the ones who are the most independent... and don't expect that friendship right off the bat. Whereas if I find someone who wants to be friends right away and get really close, and adds me on facebook, it's too much.” (I: A: 2013).

An interesting finding was that many attendants seemed to frown upon the clients whom they perceive to use their disability as a social "crutch." This attitude amongst attendants first appeared a problematic ableist outlook, but after careful examination it was found to be something rather different.

Attendants expressed particular distaste for interacting with clients who allow the category of disability to define their existence, or who treat their disability as a tragedy. One attendant shared that "there are some people at this job that just define themselves as that person in the chair, which makes it really hard. ...We do play a role in defining who they are, and definitely society does as well, but I think the only way they can come past that is if they don't define themselves as that either" (I: J, 2014). This was a common sentiment among attendants, which would appear at first blush to be rather oppressive in suggesting that people with disabilities simply need to get over their condition. But the attendants who expressed this view were mostly senior staff who were involved with the community and very forward-thinking in their conceptions of disability. These attendants also stressed the importance of social equality in their relations with clients.

This research found that attendants did not push the clients to embrace this kind of "sick role" where their disability is foregrounded. Quite the opposite is true: there was tacit agreement that "playing the disabled card" on attendants and other able-bodied people perpetuated a systemic social inequality and paternalism. One attendant's comment is particularly indicative of the ideal way in 
which attendants would like to have their relationship to the clients seen and treated: "My favourite people to run into when I'm out with someone [with a disability] is not the people who smile at us and say hi but the ones who ignore us. If I take an elevator with someone and they just do their own thing, they're not bending over backwards to get the elevator button for us, that's what I want" (I: A, 2013).

The culture established here is one in which disability must fade into the background of that interaction, and no longer be the master-signifier through which the attendant gives meaning to their interactions with the clients. One senior attendant described the transition from feeling a paternalistic moral duty toward clients to coming to understand their ethical position thus:

"I feel in the beginning, people with disabilities get put up on a little pedestal. And there are times when they do need to cut in line, and they do need this, and they do need special access to things. But now I'm starting to realize that everyone just thinks that they're these little fragile people and they're like "oh yes, yes just cut in front of me in line, oh yes, yes, just do this, and oh sweetie, I'll get you all this stuff, it's ok." and I'm like no! They're just in a chair, whatever, just let them do their thing. Don't cut in front of me, don't do this other stuff - it kind of strips away the condescension. And when I first started I probably would have been like that, too. But that's what this job has been, it's like whatever, they're just in a chair. They're the same, they're just in a chair, they might have a speech impediment, whatever. I'm just going to be your arms and your legs and just do things you can't do and that's it." (I: A, 2013).

What this attendant has described is the normalization of disability and the breaking down of its categorical significance. That takes place with experience and personal engagement in not only giving care, but in thinking about the task of care itself.

As the previous section mentioned the program structured as a kind of transition for the clients, it should be clear by now that another transition takes place, and that is of able-bodied people coming into disability culture. A key finding of this research has been that attendants learned and engaged with disability culture in entirely new ways that were unprecedented in the context of their lives. Every attendant interviewed or encountered in participant observation who did not have prior engagement with disability or disability culture, expressed that their views on disability had changed radically 
through their experience of working at Attendant Services.

"There are definitely changes. The main thing that I noticed is that people are really condescending towards people with disabilities, and I know I was like that before. Now when I treat someone with a disability as normal, like I tease them and make fun of them, people will look at me as a horrible human being. I think that's the biggest thing that changed. When I first started out I was still calling them "handicapped" and other ideas about their sexuality I wasn't really aware of, and all the stereotypes... It wasn't malicious, I just didn't know. They're disabled and not handicapped? And they're happy? I kind of had this image of them as sad, well not sad, but they're aware of how we think of them, that they must not have a very good standard of life. ... Now I notice it in everyone else.”(I: A, 2013).

This quote highlights not just that changes occurred, but how they occurred.

Indeed, a significant finding of this research was that attendants often identified with the oppression and barriers faced by disabled people. The attendants identified this way not for ideological reasons, but out of the experience of having faced material barriers of having to deal with a disability on a daily basis. A common story that came up was one of attendants not only feeling outrage at an inaccessible transit system or architecture, but in actually saying they felt disabled by it. One attendant shared "I remember going to Montreal with [a client], and it was just a nightmare of inaccessibility, and it made even me feel like I was on unequal footing with everyone else. Because I was having to take the same paths as him and use the elevator and wait in line, and I was like holy crap, even though I'm fully able bodied I don't feel like an able-bodied person right now" (I: A, 2013).

As much as the attendant and client are cast as oppositional roles in a binary relationship, it is important to foreground the fact that they are often sharing the same experiences from different angles. Furthermore, the experience of adversity that comes with disability is felt in no small part by the attendant in much the same way as the client, the one actually having a disability, does. It is not an exaggeration to say that sometimes experiencing this kind of adversity can, on occasion, be even more jarring to an attendant than a client, simply because the non-disabled attendant has never felt that oppression before. What became abundantly clear in this research was that Attendant Services provides 
an opportunity for building empathy and understanding, not just exercising it.

\subsection{Embracing Vulnerability: A Positive Culture}

Fundamental to a culture of resistance is the development of a positive self-image. From Steve Biko's famous slogan "Black is beautiful" in apartheid South Africa to the reinvigoration of Irish folk tales by the Irish literary geniuses during the times of Sinn Fein and Independence, reclaiming an ontological high ground from which to fight oppression has often involved the alleged inferiority of an oppressed group being reimagined as strength. The disability literature has struggled with the implications of this: it is no easy task to recast a degenerative condition or one which does not allow one to share in the same forms of pleasure as one's peers as positive. A positive self-image is difficult to develop around something which appears to most as objectively disadvantageous. But a positive selfimage should not be confused with the enjoyment of one's condition, which in this case would be perverse to suggest. This positive self-image is in thinking of oneself as equally human, equally part of nature, and deserving of dignity and respect as such. It would be folly to confuse a pleasant or painless life with a worthwhile and meaningful one, or to even assume that disability completely bars one from having a relatively pleasant and painless life. The following quotes collected from interviews display this culture in action:

"I think it can be positive, I kind of underscore the "can be" in that statement, also recognizing that, I'm not trying to sugarcoat the experience and say it's something that it's not because there are challenges, obviously there are. There's violence and there's trauma, you know, symbolic violence and symbolic trauma that's associated with the day to day experience that's very hard. Often I find that is an incomplete narrative, and we tend to only focus on that one narrative. And 
so the positive aspect, if I can use such a word, would be that I think what disability has offered to me, what it has meant to me, not just for a person that lives with a disability but I think for all of us, I think we all share in the making of disability. People would say that we all get disabled eventually, but not in that way, but in the actual way that we all make its meaning, we all construct its meaning every single day. I think it offers us alternative ways of being in the world, another way of existing. I think that other way of being opens up a space for multiple subjectivities, just multiplicity in general. The fact that I can still count as a human being without necessarily walking expands this notion of human, like what it means to be human on like an ideological level. Even on like a very practical every-day level, the fact that I do my assignments orally I think gives me a different perspective, it gives me a different vantage point. We can't all be the same, we can't all move through the world and understand the world the same. And the fact that I don't walk and the fact that I get weaker when I get older, that is certainly a gift in many ways to the world because again, as I said, it opens up the possibility for another way of being human, another way of being whatever, another way of being sexual, another way of being intimate, another way of being fun. It expands these categories and concepts that we have in our minds and we understand them in very normative ways, I think it opens up those categories such that there's more to explore and there's more to enjoy and live through" (I: H, 2013).

Among the clients who had spent several years in the program, literally all of the respondents

expressed having a positive self-image of their disability.

I think that my physical disability is positive in the sense that it has made me more accepting of others, and I'd say its made me more socially aware, because I've had to be. It's also helped me develop my interpersonal skills because I have to rely on other people for care. ... It's made me a little more level-headed because I'm able to put things in perspective like academia and other things because its not necessarily my sole concern. It's formed me as a person, my personality I think, it's forced me to be more outgoing because I need help, it's definitely made me more polite because I'm always dealing with people. (I: D, 2013)

Another client explained it in the same vein:

"I'm pretty good at being able to see things from many different perspectives, and I think that is hugely attributed to my disability, and I wouldn't trade that, for sure. If you asked me if I could walk tomorrow, I would only accept it if I knew my personality wouldn't change at all, and I don't think that would be possible. (I: E, 2013).

That this respondent candidly rejects walking as an intrinsically a superior and desirable way of being is telling of a very deeply cultivated resistance. Far from being perverse and fantastical treatment of impairment, this respondent is a shrewd realist in how they confront and challenge ideology at the basic level of what is deemed a desirable way of being. This kind of response was ubiquitous when asked if 
people saw their disabilities as positive. Another respondent replied:

"Absolutely, I mean how can it not be? I think that I am who I am because of all the experiences that I've had. And I like who I am, I really do. The way I've come to see the world, the way I've come to interact with people I think is directly linked to my lived experience as having a degenerative disability. I see the world in a very different way, in a radically different way, and I owe that experience to disability. It's certainly played a very important role in my life and I've definitely come to embrace that, one hundred percent" (I: H, 2014).

Another client, who had spent a much shorter time using the program told me that anyone who claims impairment is a positive thing, or that they embrace their impairment, is lying. This client's opinion was not widely shared, but represents a diversity of views. However, the work of resisting able-bodied norms should not be dismissed because it would belittle and trivialize real acts of resistance. This positive culture is not one of delusion, but it is in a very practical sense, a powerful form of resistance enacted by clients and attendants every day to elevate their lives and to reclaim dignity and humanity.

Ultimately, this praxis is rooted in embracing vulnerability. One former client expressed it thus:

[My disability] kind of allows me to get closer to people. Like, maybe that's a problem that people are more comfortable around me because I'm a little disadvantaged or whatever, but I can have some really interesting conversations with people, and I don't have to box myself in to a particular way of thinking or being. And I have a lot of the privileges of an able-bodied person, as long as I take advantage of the financial help that's at my disposal, and I continue to surround myself with people who not only help me but, you know, better me, then it's all good. And it's a struggle, but there's always a work-around. I also think I've been granted a way of looking at things that I probably would never have had if I were not disabled. It's always going to be hard, but at least there's no lack of substance. (I: C, 2014).

Embracing vulnerability may appear to be a platitude, but if it is taken more seriously it is a powerful political position. There is no way to have a frank conversation about disability without taking the fact of vulnerability as a starting point.

What another client shared represents a general attitude which the study uncovered: "I realized young that this is the way it's going to be, and has been and will forever be. I accepted that and I moved on, and now I really don't think twice about my disability. In a joking way, I think it's great because I 
never had to do fun-runs in elementary school" (I: I, 2014). This kind of acceptance is perhaps the most honest way to approach disability. Even more significantly though, this study locates a general consensus that being able to joke about one's condition and about disability in general was a way to create a positive space. There were multiple instances with different people, both clients and attendants, where the idea of basing something like a TV sitcom on Attendant Services was suggested and talked about at length (Interviews \& Fieldnotes, 2013, 2014). There are obvious pitfalls to embracing this too enthusiastically and uncritically, but it does speak to the repressed nature of disability issues when it is taboo to joke about them. Therefore, laughing about something which has been classified "a tragedy" may seem taboo to some, but it is a way people have to liberate themselves from the so-called "tragedy" of their condition. Attendant services, in this way, creates a safe space of respect and friendship where this taboo can be broken.

The program, as expressed by clients is cast, despite its flaws and limitations, as a positive experience that adds to their independence rather than taking away from it. This research suggests that experiencing and constructing disability in a positive space and on a basis of social equality with attendants, creates an opening for a view that accepts that impairment can add something and enrich the lives of individuals and communities. 


\section{Chapter 6. ANALYSIS}

\subsection{The Myth of Independence or The Case for Social Equality}

This ethnography has found that the "independent" aspect of independent living is far more complex than helping people be autonomous and productive by providing functional bodies to help them. Independence is not simply equal to autonomy, but requires a culture of social equality and respect for difference. This culture "cultivates" a world in which people with disabilities can meaningfully participate. Independence, and independent living for that matter, can only exist insofar as the conditions for interdependence and mutual recognition have been cultivated. Independence requires that a person can get help - help can only lead to independence if it is not charity - charity can never be relied upon as help, because by definition charity can never be deserved by someone requesting it (Bickenbach 1993: 196). Help can only exist in a relationship of interdependence, which requires relative equality between parties to be established first.

Achieving equality within the attendant-client relationship is not simply the concern of the client, but of the attendant, whose position is affected by their precariousness as workers. Perhaps the most clear bias I discovered in myself during this research was inherited almost directly from a faithful reading of disability studies literature. This is simply to say that not much is said in any considerable length or depth about the role and work of paid or unpaid care-givers - they are not the protagonists of that literature, and unfortunately have been often cast as antagonists or treated as incidental parts of the 
story (Shakespeare, 2006: 193). When they are referred to it is often negatively. This is understandable given the history of paternalism in "care," especially in its institutionalized forms. This perception of care, however, creates a skewed understanding of the disability economy in which care is instrumentalized and robbed of its human aspects. If this is the starting point for the political-economic relationship between attendants and clients, then there is clearly ground to be gained for attendants as equal partners in the relationship.

The way in which the attendants do their job has a large influence on how a severe disability is experienced and constructed. The working conditions of attendants also influence how attendants relate to disability. If attendants are subordinate to clients, it may give clients more power, but as a result it will prevent people with disabilities from being recognized as equals by attendants. The client will still remain an objectified other to the attendant as long as the power relationship is unequal.

The Independent Living movement that followed the revolt against the institutionalization of people with disabilities reacted by reducing the role of attendants, in theory, to mere instruments of self-help. This kind of characterization renders care work invisible, which is lacking in foresight because it is

"far more profitable to view economic dependence as biologists view ecological dependence: we are all involved with one another. Life is interdependent - and the most fruitful understanding of it can come from examining degrees and conditions of interdependency, rather than from isolating... a dichotomy between the dependent and the independent"(Rein, 1965: 87).

Recognizing the interdependence and shared situation of the attendants and clients is a first step toward equality. The task is then to mitigate or eliminate conditions of dependence by reorganizing the social arrangements which play a large role in exacerbating and creating both vulnerability and dependency. The revolt against institutionalization was an important step, but addressing the marginalization of care workers is the next. 
Care is indispensable when it is understood that dependency and vulnerability can never be fully eliminated. (Goodin, 1985: 192). It is here that the role of the attendant needs to be explored in depth as to how it is constructed as an ethical response to disability. This analysis follows the suggestion and argument of Tom Shakespeare that the non-disabled cannot be written off or treated simply as antagonists in understanding disability, and furthermore that "carers" must receive due attention as actors shaping the experience of disability (Shakespeare, 2006: 180, 186, 189, 193).

"In dramatic contrast to the total institutions, which strip people of their identities (Goffman, 1961), the helpers in this study see and assist in the accomplishment of individual identities for the people with disabilities with whom they are involved" (Taylor and Bogdan, 1998: 254). The findings of this study are in agreement with Taylor and Bogdan's findings in "the social construction of humanness." Of course, the reverse is also true: the people with disabilities in the study play a large role in forming the identities of their care-workers. Furthermore, the category of disability is transformed for both parties through their interaction and relationship with one another. Here, the social nature of disability grants the possibility of opening spaces wherein disability is not experienced as oppression, but as difference. The conditions of this "opening" can be better understood through digging into the political economy of the attendant-client relationship.

The "disability" economy is a central aspect of "disability" culture, and the separation between economy and culture, as well as the compartmentalization of a disability economy and culture, should here be firmly rejected. Not only does this economy ostensibly function on daily human practice, it not only creates, but necessitates the creation of a culture arising from these activities. This culture could be seen as a tapestry of the complex interactions and mutually constructed experiences of disability, and this is directly affected by the influence of policy on these relationships. The policy is, in this case, the structure of the attendant-client relationship under the rubric of Independent Living and client- 
directed care.

Without the development of an autonomous culture of resistance by disabled people, the Independent Living movement would not have occurred in the first place. The culture of resistance effectively changed the political structure of care, which had an important effect on the disability economy as well. The disability economy became far more consumer-centred and demand-oriented. The problem here is that even though consumers (people with disabilities) shape the marketplace and have the power to decide on how their care is delivered, being a consumer in the first place is still

premised upon the having financial resources (Albrecht, 1992: 291). While most analysis of this economy has centred on the recipients of care, the role of the attendant as a worker is vitally important to understanding the real conditions of Independent Living.

\subsection{The Dialectic of the Care Worker}

The central contradiction in the role of the attendant is the obligation to perform "caring" labour while simultaneously experiencing alienation. Not only do attendants sell their labour and are divorced from it and made dispassionate toward it, but they are also put in a situation where they are selling care - something which, by definition, cannot be treated dispassionately. In this way, the attendant is alienated from their genuine engagement with another human being - an engagement which is necessary to their job as a carer. The "product" of this labour is made and shared both by the attendant and the client.

The mutual production of care was described by one respondent: "when I was at Carleton it was much more like, my independence was a mutual task that we both derived satisfaction from. So my independence was related to your independence, because I became friends with more or less all of the 
male staff'(I: F, 2014). The crucial distinction is that the experience of disabilities is not solely the experience of the people who embody them, but of those who share in the experience through helping or interacting with them. As Paul Higgins explains, "disabled people, family members and those unfamiliar with disability may experience disability in conflicting ways. That conflict itself becomes part of experiencing disability" (Higgins, 1996: 149). The fundamental fact of the care/disability economy is that through this economy, its members share in the experience and construction of disability and care.

In contrasting the Carleton and Algonquin Program to Independent Living in the broader community, a respondent noted that "there they're concerned with your independence, but it's much more like how can I be a biological robot who facilitates your independence within these confines"(I: F, 2014). On one hand there is the alienation of being a kind of "biological robot" who functions as a self-help tool for the client, and on the other hand there is the vision of being a worker who participates in a joint production of care. The contradiction of the attendant again is that while genuine engagement with care cannot be bought and sold, genuine engagement cannot be divorced from the work, which is sold. Even if the worker is ostensibly a "biological robot," it does not erase this contradiction, but only pushes it deeper.

The difference between workers at Attendant Services and those in other Independent Living environments seems to be in the presence of a higher authority in the form of a head office which can effectively control how attendants engage with policy on stricter terms. A client who moved on to one of these residences described them as more "policed and policied" (I: E, 2013) than the program at Carleton. Another former client described the care in the community as being a more efficient, but less "human" form of care (I: F, 2014).

In the Carleton-Algonquin program, the distance of the management and the looseness of the 
policy and control contribute somewhat to making the care relationship less formal. One client said "it still has negative aspects to it because it's less formal, but the informality of it made it feel less like an institution"(I: E, 2013). An important distinction to make is that informality, in this case, denotes a workplace that is less tightly governed. The debate on the economy of informal (unpaid or less-paid) versus formal care (paid) is an important one, but obscures a line of distinction in the debate by focusing on fiduciary concerns. Formality is associated with efficiency and a kind of custodial caring for, and informality with perhaps a more humanizing experience that involves not just caring for but caring about. The reality is that both of these aspects need to be balanced together. It is worth considering that these two aspects, like care and professionalism, do not exist on a sliding scale.

Formality is an adherence to a set of procedures, but the crucial distinction that needs to be understood is that what is "informal" implies going beyond or outside those procedures and not necessarily against them. Therefore, informality can also be the fountainhead of new procedures and new solutions, and not just a set of uninformed practices. Ideally Independent Living necessarily presents a challenge to the very idea of having rigidly formal structures in care by placing control over the services in the hands of the clients.

The position of the attendant as a worker is not properly represented by the informalityformality debate because, as this study has found, the working conditions of the attendants are the living conditions of the clients. It has been commonly represented in disability studies that when more rights are given to carers, it is at the expense of the cared-for (Shakespeare 2000, 2006: 189). What this study has found is that creating better conditions for the interaction of attendants and clients, though it will never remove friction from that relationship completely, will help both parties overall and in general.

The argument that untrained support workers are better able to provide services on a basis of 
equality because they have not been inculcated with the paternalistic attitudes of professionalism is very important, but overlaps dangerously with arguments for keeping these people low-paid (F. Williams, 2001: 482). The Attendant Services Program shows this very point of tension in the debate, because while "untrained" workers are likely to be less paternalistic in their attitudes, they suffer from marginal working conditions. To understand how attendants are marginalized, one must understand the consequences of care being sold as a commodity.

The "social distance" that separates people as independent individuals can collapse in the relationship between attendants and clients when either party identifies too closely with the other. As commodity relations recreate the gap to re-establish that social-distance, it alienates both the care worker and their client while simultaneously protecting them from the over-proximity of the other. A more intensely commodified care means greater social distance and greater alienation of the careworker and their client from the mutual task of producing well-being. The well-being produced is a narrowly functional one in the context of these transactional relations.

In theory, reducing complex human interaction to the logic of economic transaction sanitizes the process of care by repressing anything outside of economic rationality or concerns of maximizing utility and efficiency. In practice the human aspect is far more difficult to repress in care work, because caring is a challenge to the notion itself that we are autonomous and self-supporting as human beings (Tronto, 1993: 134). At Attendant Services, the attendant has less recourse to policy which allows them to maintain social distance without their own active personal intervention to establish that distance (Interviews, 2013-2014). As a result, the attendant at Attendant Services has no choice but to engage in order to disengage, instead of simply invoking professionalism and using policy to excuse themselves from difficult situations.

The attendant at Attendant Services has less space to invoke the "argument of scarcity," that is, 
for an attendant to tell a client that they do not have time for them when they may in fact, because the community is held together more by social ties than by monetary ones. They are more likely to do more things and to indulge clients in things outside their immediate needs if their time permits, because they have little formal institution to hide behind. The function of the institution of attendant care in the broader community is in making the time of the attendant into an exclusive good, that is, in using policy to make their help a scarce resource. This applies even if help is available more often; the attendant still has recourse as a more detached professional to invoke a scarcity of time that their workplace has institutionalized through strict scheduling. The attendant at Attendant Services is more flexible because they are made more amenable to being flexible by the social ties in their workplace they become friends with people and so do more non-essential things for them. Not just friendship itself, but the ingrained expectation of friendship can often facilitate a different kind of work and work interactions.

The cooperative and task-oriented nature of the client-directed care provides a foundation for interactions in this work relationship and structures the work in a certain way. Within this broad scope the "product" of the care work has as many variations as there are combinations of people in the program. The attendant and the client together author the "functional independence" of the client based on that individual's needs and the way in which the attendant works to meet those needs and, therefore, the result of this complex interaction is not an unchanging or standard product. The interaction is complex in the precise sense that it resists standardization. By way of analogy, if one attempted to get 200 pairs of people to assume opposing roles to their partner and then give them a vague and general script for a conversation, it should be unsurprising that all of these conversations will end up happening quite differently, all with their own idiosyncrasies, though they reference the script. What this kind of exercise would suggest is that it is impossible to suggest the means of giving care cannot be effectively 
bifurcated from the end of receiving care, because in this kind of work, with people's well-being as the product, the ends of this process arises quite clearly from the means in a way which makes the two impossible to treat separately.

The problem is that universal morality congratulates attendants for their charity a priori their behaviour, and mourns for the suffering of clients, a priori their quality of life (Tronto, 1993). That the means is inseparable from the ends is a hallmark of contextual morality, whereas universal morality, to put it crudely, argues that the end justifies the means. To put this into practice, the institutionalization of all people with disabilities was deigned as moral by such a "universal" framework. The attendant Code of Conduct displayed earlier represents a universal ethic, but as this thesis has shown, is inept and silent in actually addressing the difficult ethical situations faced by attendants every day. Applying universal morality to the attendant-client relationship skews the actual political dynamics within it, and moreover nullifies the importance that these dynamics hold in the production of functional independence. To put it bluntly, within the framework of a universal ethics, if there is then nothing new one can learn from this relationship, it is because under this framework literally nothing can be known about it. All that can be known is whether it creates the desired end or not.

It is this kind of logic which sustained the institutionalization of disability as a worthy moral project. Indeed, in addressing impairment, creating a universal ethic to follow is the very definition of "institutionalization" itself. It is not surprising that many of the clients who have moved out into the community Independent Living residences with much tighter control and regulation of policy describe them as maintaining the function of Independent Living, while at their core being "more or less just a nicer form of institution" (I: F, 2014). Independent Living on some basic level could be construed as the ability, however limited, to be treated under the rubric of a contextual morality - that is, as a human being with particular needs, by another human being who recognizes those needs in their 
context. In care, an unflinching universal morality can dehumanize people by attempting to treat them outside of their context and circumstance.

There is nothing more fundamental to care than situational and relational understanding of an other (Tronto, 1993). The necessity of situational and contextual ethics needs to be acknowledged as an integral part of Independent Living. Moreover the consequences of institutionalizing Independent Living itself needs to be studied further, because even though it is a move in the right direction, problems will ultimately arise if it is treated as a rigid and unadaptive system.

\subsection{The Role of the Attendant Revisited: A Revised Worker's Manual}

What follows in this section is a summary of the insights gained through the research, framed in the practical terms of how to be an attendant in a commodified relationship. It has been written to fellow care workers. It contains recommendations, but it is also an analysis of the ideal attendant which became apparent after studying the culture of this particular economy of care. The ethics described here

are already embodied by most of the workers in this program, most of the time; the aim here is simply to further articulate them.

In order to understand the complexities of being an attendant, one must be able to follow the logic of the distinction between giving charity and respecting a right. By definition, no one will ever earn the right to another's charity, and therefore a charitable attitude cannot be sustainably employed to meet the end of satisfying the right of another. Many do not become successful attendants, for in attempting to be an attendant, people find a frustrating gap between their benevolent intentions and their mundane duties as a worker. It is largely a failure to fully mark the distinction between doing good things out of kindness and doing good things for a pay cheque. For people who are successful in 
the job, understanding this crucial difference seems to take, on average, a year (fieldnotes 2014, interviews, 2013, 2014). This may serve to explain the high burnout rate among attendants and the concomitantly high turnover rate. But this failure is understandable in the context of a first encounter with disability, because

"It is inevitable that they should be strongly moved by all this. The emotions of man are stirred more quickly than man's intelligence; and ... it is much more easy to have sympathy with suffering than it is to have sympathy with thought. Accordingly, with admirable, though misdirected intentions, they very seriously and very sentimentally set themselves to the task of remedying the evils that they see. But their remedies do not cure the disease: they merely prolong it. Indeed, their remedies are part of the disease." (Wilde 1997)

Though it may seem counter-intuitive, in order to break the endless cycles of commodification and to resist the hollowing out of "care," it must be acknowledged that the attendant-client relationship is already commodified. Any attendant who does not understand their task from the perspective of a worker immediately places themselves in a higher position: in a position from which they can charitably give to those whom they see as disadvantaged. Though having the most genuine intentions, they may in the end become something as disingenuous as a "friend for hire" who is no longer for hire; being a worker who cares is, on a fundamental level, a different ontology, not to mention a radically different ethical framework.

Rejecting the commodity basis of the relationship is also a sure way to be exploited as a worker. If the wage labourer does not recognize that they are in fact selling their labour, then they have no basis to judge when their labour is being undersold or overworked. Indeed, this program is held together by a manner of surplus extraction that is tied to romanticized and gendered notions that see caring not so much as "work" in the proper sense, but as an act of love. This should be resisted by workers, as workers. This is not to say that one should avoid caring too deeply or being a friend, but rather be cognisant that caring is work, and friendship is not given. 
The problem of the care worker is not one of efficiency or productivity but one of recognition. That is because the product of care cannot be divorced from the process of care, a process which is consistently gendered, racialized and rendered invisible as work. The attendant should not fool themselves into thinking that giving better care is necessarily a matter of efficiency and productivity: it is a matter of recognizing care as a process and a product (Tronto, 2010). Care is both the means and the end of a relationship with another person. An overly formalized structure of care can lead to an arrangement where care workers do not serve clients as much as they service them. The servicing metaphor is taken from Paul Higgins (1992: 153), who explains "to service an object is to fix or maintain it," and that the role of most societal institutions that deal with disability is precisely to service rather than serve people with disabilities. The kind of care that servicing represents is the care of an object, not of a human being.

On the other extreme, seeing oneself as a "friend for hire," though it may at first blush seem to describe the situation of attendants and clients somewhat, is either extremely naive, or extremely cynical. To put it bluntly, friendship, by its very definition, cannot be commodified. The distinction must be made that it is not impossible that something genuine could come of a commodified relationship, but for there to be a genuine engagement, it must be honest about the conditions from which it emerges. There are a few potential pitfalls in this process.

Disability may be seen by some as a personal tragedy, and this is completely valid to a certain point. Many able-bodied people intuitively embrace this narrative because in their attempts to empathize with disability, they confront an existential anxiety arising from the cracks in the fantasy of their able-bodiedness. But this idea of disability as a perpetual tragedy offers people with disabilities to be seen only ever as less than or more than human - never simply just human and hence never equal (Oliver and Barnes, 2012: 118). Furthermore, defining an impairment as always already a personal 
tragedy tends to make disability experienced as personal tragedy as well (ibid 14).

Experienced attendants at Attendant Services, knowin disability and the disability economy from the other side, eschew this understanding of disability as personal tragedy. Their work is simultaneously romanticized as being a great act of love helping others in need, and devalued as actual work by the very same logic - if it is an act of love and charity, why should anyone be paid well for it, should they not do it just out of virtue? Disability as personal tragedy also robs the attendant of any but the most perverse satisfaction they could get out of helping another person, since the task of care is mutual; if a client is unpleasant and dwelling in the tragedy of their condition, they are by default forced into being a saviour, whether willing or not, who cannot possibly deliver salvation.

The view of disability as personal tragedy does not build friendships or amicable work relations, it creates another whose state is one of perpetual grief, and whose only plausible fate is a bitter dependency. Attendants have been shown to resist this just as vigorously as the clients. Otherwise, if the attendant does not resist this kind of perverse script, they accept the oppressive cultural narrative of their task being one of well-meaning and altruistic futility and perpetual failure.

The task of the attendant is in constructing and maintaining a positive culture of social equality and resistance toward disabling norms, ideas, relationships and environments. One key aspect of this is resisting the disability-as-personal-tragedy narrative. The work environment and work relationships can create or destroy a positive culture around disability. There is no formula that can completely eliminate the friction in these relationships and, furthermore, some friction and conflict is necessary in order to establish proper boundaries in particular relationships where the invisible hand of policy does not evenly reach.

Conflict can also create understanding, because after all the clients and attendants are in a constant state of negotiating their relationships. What the experience of Attendant Services highlights is 
that this process of negotiation can be an incredibly positive thing, even a profound thing, for both parties. That clients and attendants negotiate does not mean that policy has failed - just that it could never completely succeed as a total ethical framework for care. Policy is necessary, but that space of negotiation between equals is what allows the category of disability to escape hierarchy and re-emerge as a difference. This kind of space has been cultivated in many instances between attendants and clients at Attendant Services, and with its uncertainties and irrationalities allows a more human experience.

\section{4 "Independent Living is Dead, Long Live Independent Living"9}

The relationship of power between the program and its clients is of great consequence and has a broader significance for policy makers. The relationship is defined by the Attendant Services Program at Carleton and Algonquin being the only program of its kind. This fact affords the Program structural power through the fact that for the clients, there is nothing better because there is nothing else. The mere fact, however, that the Program is the only available option does not stop people contending, quite logically, that there could and should be other programs like this one at other universities. One interviewee explained "I got lucky because the program I wanted to take was at Carleton. But there are so many people that you ask them why they went to Carleton and that's the reason. You should be able to get to choose where you get to go to school, and it shouldn't be dictated by where you're physically able to live" (I: E, 2013).

Some within the program have suggested that this model is not possible at other universities due

\footnotetext{
${ }^{9}$ As someone who is involved with this program, the main goal of my analysis is to point to new solutions and possible problems. This is meant to be an honest appraisal of the strengths and weaknesses of the program and how to address them. As such, the analysis and recommendation aspects are intermingled in the following section.
} 
to a lack of expertise, lack of funding and lack of people who "care" enough (F: 01 2014). It is quite natural to want to protect the Program at Carleton and Algonquin as it has been difficult to build and maintain its funding. Where this argument falls apart is that there are not people who care enough and are committed enough to make this work elsewhere, and furthermore that expertise cannot be shared. Funding is a very real obstacle, but encouraging the political will to fund and encourage accessibility should not be underestimated or be dismissed as an impossible horizon. The yearly cost of the program at Carleton, though an exact figure was not obtained, is relatively little considering the intrinsic value of what it achieves.

Indeed, at their root, these are reasonable contentions, but their effect on the power structure of the Program itself is rather more insidious. This kind of rhetoric fills in its own logical gaps by referencing a disturbingly Ableist ideology. As Oliver and Barnes note, "Individuals with impairments are socialized into viewing themselves as worthless, useless, of lesser value than their non-disabled peers and therefore completely dependent upon the goodwill of others for their very existence and survival" (Oliver and Barnes, 2012: 138).

The Program, in a very significant way, continues and reinforces the very structures it is trying to dismantle. Since it is insisted upon that no one cares enough to do this anywhere else, and there is no political will for funding, the clients are put in a position where the Program is sold as their only hope, and furthermore they should not expect political change because nobody really cares about them outside of it. What is Ableist in this is not that it recognizes that the political conditions for Independent Living are contingent, but that at the end of the day, this particular set of caring able-bodied people are the only ones who can create the conditions. This rhetoric seeks to take the power of political action away from people with disabilities and to encourage a kind of quiescence - an attitude of "we can't win so why bother?" toward seeing access as a right. 
Fortunately, many are not discouraged by this assertion, and frankly do not believe it. One client interviewee said the following: "In a bigger sense, they dictate what you can do based on what's available. There's nowhere else in Ottawa that I could live right now because there's only a handful of accessible apartments and they're all taken because the demand is way bigger than the supply"(I: E, 2013). The demand for new programs also has to come from their would-be clients, and when supply is not meeting this demand, it has become a political issue. An inefficient market requires intervention, but the intervention it has so far warranted is the maintenance of that under-supply, at the expense of the ability (not to mention the right) of people to live where their basic needs are met. The Gordian knot here appears to be that the gains made in the Program at Carleton and Algonquin must be protected, but also that the structure of this program encourages quiescence, in the final instance, to the ideological assumptions of Ableism: that able-bodied people hold the fate of people with disabilities within their hands, and paternalism becomes again justified. Moreover, this ideology would make it appear that the only protection from the destructive and silencing paternalism of institutional care is nothing but the goodwill of a few enlightened, able-bodied people.

The final message to the clients is "do what you want because you are independent, but always remember that your independence is dependent upon us." To cut the Gordian knot is then to turn this logic on its head: "always remember that your functional independence is a product of interdependent relationships, but in the end you are in a political situation where your belief in your own independence affects what is possible." It was not institutional paternalism that liberated disability as a concept from its own suffocating definitions; it was people with disabilities who threw off institutional paternalism and took control of their situation on the grounds that it was a political one.

The generalized distrust and disregard of management at Attendant Services and sympathy between clients and attendants is the resistance that keeps not only the spirit of Independent Living 
alive at Carleton and Algonquin, but its daily function as well. The transformative culture of Attendant Services has not emerged as the result of stability, well-enforced policy, and strong and present leadership: it has been the result of something like a constant situation of emergency where Independent Living is perpetually on the brink and policy and management seem impotent to do anything to save it. All that is left is the dead saviour, the absent paternal figure, and the attendants and clients who have no other alternative but to make it work; that is the honest reality.

As a recommendation, the first thing that could make the Attendant Services Program better is establishing more programs like it. An ex-client in this study put it in the following terms "And together through everyone's ideas and everyone's abilities, all of the campuses that are accessible ... all of them together will be able to collaborate and build a good foundation for what an attendant services program should look like. Because the one at Carleton is amazing, but definitely flawed" (I: E, 2013). This was echoed by many other clients as well as some attendants. 


\section{Chapter 7. RETURNING TO THEORY: TOWARD A THEORY OF IMPAIRMENT}

\subsection{Cutting the Gordian Knot: In Defence of a Social Model}

As E.P. Thompson put it, research should bring theory and evidence into dialogue. To return to theory from the discussion of the evidence, I find reason to confirm existing theory, as well as reason for the alteration of that theory.

First, the distinction between disability and impairment remains a useful one, because if disability is treated as a social construction it allows disability to become political and therefore to be a site of change and possibility. Impairment, as the mean physical fact behind disability, is no longer a site of political contention, but remains as the tether linking disability to the material world in an unassuming manner. This social theory should, however, be reformulated to clarify the role and function of impairment, because it is, in reality, hardly the unassuming tether as it is presented.

Indeed disability, as a social and political category, is an important tool for developing understanding and for political change. Disability should be seen as an historically situated category; disability can be thought of as the modern condition of impairment which accompanied industrial welfare policy and scientific governance. In this sense I am in agreement with much of the Social Model literature on the category of disability. The much more difficult question is then what to do with impairment as an "unsocialized universal" concept (Tremain, 2002). The call has been to create a social 
theory of impairment, but to do so would only more or less replicate the social model of disability while calling it by a different name.

Impairment seeks to grasp the material reality of disability: the fact of limitation itself. The problem lies in the fact that limitation cannot be defined outside of a cultural or geographical context. Therefore, this formulation leads back again to a totalizing kind of social model that does not adequately address the material. We are left again with a conservative rejection of the ability of social constructionism to liberate us from issues arising from our material condition. The solution is not to hand Ableism rights to the underlying facts of life, but a deeper examination of what impairment is, or rather of how a distinction between disability and impairment can be drawn.

Tom Shakespeare offers the strongest critique of the (British) Social Model's separation of disability and impairment and the consequent elision of the material. He presents the idea that universal access is simply a dream that will be impossible to graft onto reality - that the social model is, in other words, hopelessly utopian. The reasons Shakespeare offers to substantiate this assertion bear a closer look. In his critique, Shakespeare insists that "numerous parts of the natural world will remain inaccessible to many disabled people: mountains, bogs, beaches are almost impossible for wheelchair users to traverse," (Shakespeare 2013: 219). This provides an interesting clue as to where this distinction may lie. For all the value and insight in Shakespeare's critique of the social model, as it has surely been invaluable to this thesis, this is seemingly commonsensical statement is ideology at its most insidious.

I should not begin by saying that I know plenty of wheelchair users who have been to the beach, as well as many nondisabled people for whom mountains are inaccessible - but I could not resist. Furthermore, I should note that Carleton University has incorporated curb-cuts for wheelchair users alongside markers of where the sidewalk begins and ends for the visually impaired, which is another 
utopian dream of universal access which Shakespeare did not think possible. These small points aside, Shakespeare's argument is nonetheless powerful and presents an uncompromising knot.

When examining the relationship between human beings and their environment, the experience is one of overcoming limitation. The logic here is that left alone in a "natural environment," a disabled person will be unable to cope; and what is most interesting is that while this is not necessarily false, it is also a complete fantasy. Here one can see more clearly impairment's appeal: it is the final material limitation which people with disabilities must face, it is the reality of their condition. But it is at the same time precisely the opposite, it is fantasy, it is a condition divorced from the context that gives it meaning.

Following Shakespeare's logic, there are many places in the "natural world" that are inaccessible to virtually all human beings. Extending this logic further, not everyone can climb Everest, and more importantly, those who cannot are not thought of as impaired because they cannot do so. Again, by this extension of logic, every human being is more or less on a scale of impairment - which is true, but misses the point of seeing disability as an historical and political category that emerges alongside the capitalist mode of production. Impairment may be a spectrum, but disability is a political category which deserves to be maintained as such, and not subsumed under a kind of "natural" theory.

Perhaps this was the kind of naive question someone needed to ask within the disability literature, precisely because it puts on display how impairment as an "unsocialized universal" plays directly into the fantasy of Ableism. Impairment then, must be understood not as the reality or material basis for the social constructions of disability, but as a category of fantasy.

To understand the full implications of this fantasy, its point of origin must be explored. Impairment is in fact a reference to a state of nature, which is a hypothetical view of what human beings would be like outside of history and civilization. This is an important point of critical enquiry 
because "to paraphrase Derrida (1976), there is no space outside such cultural practices from which nature can be objectively known" (Braun 2002, 15). That is to say, these hypothetical states of nature are usually better reflections of the operating ideologies of the time than of the economic and political organization of any society that actually existed in the past.

Shakespeare's work, as such, can be mined for these ideological assumptions that do not so much reflect material reality as they refract ideology. Shakespeare's statements provide some remarkable artifacts which demonstrate a way in which disability is read in relation to nature. Take for example his statement that "even if it were practically possible, it would defeat the very idea of wilderness to create roads and other access facilities to unspoilt and inaccessible landscapes" (Shakespeare, 2006: 46). Nothing in this statement is apparently false, but however, in this seemingly innocuous claim and fair treatment of fact there lies ideology, in the purest form. Wilderness is, as Shakespeare himself points out in this statement, an idea and one that can be spoilt. It is well worth asking why exactly disability and accessibility spoil the very idea of an "untouched wilderness" as a hallowed natural space. It seems that we may have stumbled upon a deeper and more pervasive spatial segregation which Shakespeare has risen to defend.

To gain further insight, one should turn to the work of influential ecological historian, William Cronon, which takes apart this very idea at length in "the Trouble with Wilderness." Here wilderness is revealed as an ideological construct:

"The more one knows of its peculiar history, the more one realizes that wilderness is not quite what it seems. Far from being the one place on earth that stands apart from humanity, it is quite profoundly a human creation - indeed, the creation of very particular human cultures at very particular moments in human history. It is not a pristine sanctuary where the last remnant of an untouched, endangered, but still transcendent nature can for at least a little while longer be encountered without the contaminating taint of civilization. Instead, it's a product of that civilization, and could hardly be contaminated by the very stuff of which it is made. Wilderness hides its unnaturalness behind a mask that is all the more beguiling because it seems so natural. As we gaze into the mirror it holds up for us, we too easily imagine that what we behold is 
Nature when in fact we see the reflection of our own unexamined longings and desires." (Cronon, 1995)

These longings and desires have long been associated with normative embodiment; that is to say wilderness is only wilderness because it is inaccessible. Moreover, the concept itself has changed throughout history: "Wilderness had once been the antithesis of all that was orderly and good—it had been the darkness, one might say, on the far side of the garden wall—and yet now it was frequently likened to Eden itself" (Cronon, 1995). The comparison to Eden is indeed significant.

What Shakespeare (undoubtedly unconsciously) reproduces in these statements is that impairment can never participate in an Edenic state, for it is always already a fallen state of being, and moreover a failure to adapt to nature. In a word, wilderness, constructed as an Eden of inaccessible terrain, renders impairment unnatural. Cronon astutely recognizes this in contending "if we set too high a stock on wilderness, too many other corners of the earth become less than natural and too many other people become less than human, thereby giving us permission not to care much about their suffering or their fate"(Cronon 1995). Indeed, what Shakespeare offers as common sense and material fact is quite inextricable from ideological notions of normative embodiment.

In speaking exhaustively about a "disabling environment," the discussion must seriously consider the form and content of utopian visions of the environment because "utopian thought... would be a necessary aspect of a theory of oppression in that it denotes what it would mean for that oppression to be abolished." In other words, one must ask "what are the consequences for impaired people of your good society,"(Abberly, 1996: 67) or in this case, of your Eden? The way in which human beings should move through and interact with the environment is implicit in any utopian view of the environment. Wilderness-as-utopia demands a particularly rugged kind of able-bodiedness of its individual inhabitants in order to survive. 
In making the point that the material matters as well as the social and the ideological, Shakespeare has perhaps undermined his own contentions by making the social model into a straw-man. Thus "conservative conclusions are drawn from what are held to be unalterable features of the human predicament: from our own inner natures, from external nature, or from a combination of the two" (Benton, 1989: 57). In the most seemingly common sense part of Shakespeare's critique of the social model, there are completely arbitrary and ideological limitations of impairment.

These ideological limitations begin setting up boundaries by alienating disability from the natural environment, effectively separating disability from a natural order of things. This natural order of course refers to a state of nature where social relations are at their most basic. These basic social relationships then are not the kinds of ethical relationships that occur in the Attendant Services Program that are characterized by care for the other. They are instead always already characterized by the exclusion and dependency of those with impairments upon their more able peers.

The relationship between attendants and clients could, from this philosophical standpoint, be seen as unnatural or even an intervention against nature. Nature then becomes the source of a universal morality that congratulates attendants for their humanity a priori their behaviour and relegates people with disabilities to a state of misery a priori their quality of life. The hierarchy is firmly re-established when the basis of disability is given back to the concept of nature which posited it in the first place. With the ethnographic evidence here on hand, one can assert that the relationship between the disabled and the non-disabled cannot be reduced to a matter of natural hierarchy: moral and ethical objections aside, it simply does not reflect nor represent reality. In trying to theorize impairment, there needs to be an attempt to reach toward some manner of universal generalization which does not end in tautology.

Disability is impairment situated within time, space and social relations: disability is impairment in modernity. The real work lies in qualifying the universal of impairment which may be 
posited: it cannot be a sloppy and unsubstantiated assertion of human nature. Impairment needs to be similarly situated in space, time and social relations if it is to be explained by social theory.

Nature invoked in politics is never simply nature: it is a specific order of things. If we accept this, a productive discussion with substantive content can be had about nature. The best resource and method available for an attempt at discerning a kind of (social) theory of impairment is economic anthropology because it rejects the simplistic view offered by "states of nature." Not only that, but economic anthropology actively counteracts this by putting on display actual lived social relations in societies that may be said to exist in a "state of nature." It allows one to see the civilized and uncivilized worlds without an arbitrary delimitation between them. Thus the economy of care that this study has focused on can be appreciated in its own context rather than against an abstract universal which always already invalidates its existence as unnatural. Economic anthropology is useful in situating what is crudely referred to as "nature" as a real historical and social situation, in order that this theory of impairment may be given an empirical basis - that is to say, to make it a theory in the first place by providing the grounds upon which it can be both proven and falsified.

Since disability is an economic category arisen from the mode of social organization of emergent industrial society, one can surmise that in different modes of production the phenomenon and experience of impairment might take on quite a different cultural significance. Here, perhaps it makes more sense to speak in terms of varying "modes of subsistence" or "modes of social reproduction" than "modes of production" as the basic analytical unit of economy. This may serve to better express the human limitations of economy.

While addressing the relationship between impairment and subsistence in hunter-gatherer societies, this should be compared to the vision of the classical economist's savage, which provides modern economics' basic assumptions about the human being's relationship to their social and material 
environment. Through this comparison it can be discerned which material limits are real and which are purely ideological. In concluding, the place of the attendant in this configuration will be discussed.

\subsection{The Crippled Savage I: The Founding of a Utopia Outside of Nature}

A good historical materialist political economy is, in many ways, the study of human ecology. It studies the relationships between human beings, human beings and their environment, and human beings and other non-human organisms. It studies the system of how these things interact as a whole to produce a collective way of being, a kind of ecosystem. It constructs a view of the world from the relationships that occur within it. These relationships, however, are not simply given and absolute, but change, develop or stay the same over time. What separates this kind of study from ecology is that the ways of organizing these relationships and these material flows of energy is not given or determined: it is contingent and political.

Not only do human beings interact on the material level, but as social animals they construct a world of meanings with and through one another, to understand and symbolize the material world around them. This system of meanings adds a whole other complex and rich layer to the raw facts of their ecology; indeed, how this ecology itself is understood is a product of the representations human beings give to their material reality, and structure their ideas of possibility within it. These representations vary across time, culture and geography, and are thus political and contested.

Politics has been described as the realm of the possible, so to have a political issue is to have a fundamental argument about what is possible. To establish a stable day-to-day economic order, the 
political questions of how relationships should be structured and to what ends they should function need to be agreed upon. It is hardly simple, as these relationships are often in flux. The political economist studies precisely the conditions of this flux, its social and material causes and consequences, and its historical path.

Economic anthropology puts on display and takes as its object of interest the different and varying forms that these relationships can take, across all manner of different times, cultures and places (Godelier, 1978: 49-51). What this discipline teaches is that when it comes to human creativity towards organizing into functioning groups that can not only subsist but live well and happily, there are far more possibilities than are often presented in modern utopian visions. Every society and social order has a vision to which it aspires, and of course the reality always differs in important ways from the ideal, but the ideal nonetheless serves an important function of guiding the hopes, dreams and energies of the people within it.

Utopian visions also rely upon these other possibilities for social organization to serve as reminders as to why this certain utopian ideal is superior. Thus, a utopian vision constructs a kind of "savage other" to which it refers. This savage other has two primary purposes: one is that the savage is the human being without all the benefits and wonders of utopia, and is completely outside of civilization. She, the savage, is not only outside of civilization, but "temporally superseded" -- cast into the past and treated as an anachronism which has nothing to do with the modern utopia (Blaney and Inayatulah, 2010).

The basic relationships and possibilities available within human ecology are extrapolated from the ways in which this hypothetical savage is said to behave. The savage lives in a state of nature, and this state of nature determines the unalterable conditions of how society grows and works. In other words, the savage in the state of nature is a view in hindsight, a fantastical version of the past built on 
the terms of the present. The edifice of the savage in the state of nature serves to maintain a political order - to stabilize and direct what is possible in present and future society.

Understanding nature as an historically changing category allows for a less superficial engagement with the material barriers that structure what is possible for human beings and their societies, and as such is vital to political economy and the project of historical materialism. Analyzing change over time in the relationships of human beings to one another and to their environment can only advocate for justice within the confines of an already unjust framework, that is, if the category of the savage and of nature is left unexplored and untested in its assumptions.

The savage state is a priori a failure of social organization for economists, and "primitive man is not quite a man because he is not homo-economicus," (Meillassoux, 1978: 129) meaning that the savage does not act according to models of economic rationality. This savage state of nature is also the repressed fantasy of absolute freedom; hence Rousseau's famous pronouncement "man is born free and everywhere he is in chains"(Rousseau, orig. 1762), and the equally famous contrasting vision of Hobbes that life in the state of nature is "nasty, brutish and short"(Hobbes, orig. 1651). Rousseau decries the shackles of civilization in contrast to of the freedom of the savage, while Hobbes cautions us towards the horrors of that absolute freedom. ${ }^{10}$ What the social contract theorists more or less agreed upon was that this savage impulse to pursue the fantasy of absolute freedom had to be constructively repressed in order to build civilization. Building on the bifurcation of mind and body established in Descartes' first philosophy ${ }^{11}$ and Discourse on Method, these theorists, among many other Enlightenment thinkers, committed to a separation between "man" and nature (Braun and Moeckli,

\footnotetext{
10 Both accounts, however, have their fatal ontological flaws. Rousseau's Noble Savage was based on a romanticized misunderstanding of indigenous North American life, and Hobbes' state of nature was less a state of nature than a refraction of his own experiences of the breakdown of law and order suffered in the English Civil War.

${ }^{11}$ The first philosophy being "Cogito ergo sum (I think, therefore, I am).
} 
2001: 117).

"Man" always already belongs to civilization and should thus be civilized by repressing his savage impulses, while nature existed as the opposite of civilization: nature is the home of the repressed savage within civilized "man," a home to which civilized "man" has foreclosed the rights by being part of civilization. As a result of this repression, nature is a fantasy and a fetish of the so-called civilized man; it is an ideal that grew out of the experience of civilization rather than the experience of nature (R. Williams, 1980). What we are left with is one of the greatest epistemological knots ever tied: human beings cannot experience a pure natural state, though the pure natural state sets the boundaries of what is possible for human beings.

The classical political economists took this separation between human beings and their environment and enshrined it within the worldview of the nascent discipline of economics, structuring theory and method for generations to come (Daly, 1996: 48-49). The economic anthropologist Karl Polanyi explores at length the foundational moment for classical political economy's enduring vision of nature, noting:

"Economic society was founded upon the grim realities of Nature; if man disobeyed the laws which ruled that society, the fell executioner would strangle the offspring of the improvident. The laws of a competitive society were put under the sanction of the jungle. The true significance of the tormenting problem of poverty now stood revealed: the economic society was subjected to laws which were not human laws" (Polanyi, 1944: 98).

With this vision, the classical political economists more or less created human beings' inner nature in the image of their theories on economic rationality. They took a specific set of work and property relations arising at a specific time and specific place and abstracted it into a universal condition and basic tendency in humanity.

Economic anthropology is necessary to this enquiry because it offers a history of economy 
which treats the founding myths of economics as just that. Therefore economic anthropology does not foreclose possibilities offered by other ways of organization by putting them in a hierarchy of developmental stages. In other words, it is a tool to free ourselves from false histories by looking at the "savage in nature" as a symptom of the present, and not a reality of the past. In this way, we can be liberated from this supposed baseline of economic logic.

\subsection{The Crippled Savage II: The Question of Technology}

To talk about a history of impairment, what are we really talking about? A limitation of human physical or mental capacity defined in relation to their environment, which implies the ability of human beings to move within their material and social world. The material world raises questions of accessing food and shelter, and the social world dealing with issues of navigating the politics of interpersonal relationships and the confines of culture and tradition. So, in a basic sense, an impairment is only an impairment in relation to a set of conditions.

The history of humanity is often told in terms of the advance of technology: the neolithic revolution and the development of tools; the agricultural revolution and the development of farming; the industrial revolution and the development of complex machinery; and the digital revolution and the development of communication technology. Reading that history backwards, that is from our present standpoint looking at the past, one could easily see the regressions in technology as creating a more impaired human condition given the absence of medicine and modern tools, transportation and farming techniques as one goes back in time. So in many ways, this history of technology is a history of humanity overcoming its own limitations and intrinsic impairment (read mortality and vulnerability) through technology.

But in reading advances in technology as historical progress alongside the history of disability 
would tell a different story: as there is more technology to help human beings, there is also more exacerbation of the limitations and impairments of human beings. As Paul Higgins explains,

"Our manufactured progress may also be debilitating. Even as we improve our lives, we manufacture disability. .... We create it on the assembly line, in front of the video terminal, and in the executive suite. We produce it on the highway and at home. We create it in far away countries during armed aggression and in our neighborhoods during equally dangerous assaults. ...We grow and produce the substances with which we disable ourselves, and we glamorize them in order to turn a profit. ... In a tragically ironic and short-sighted way, making disability is big business -figuratively and literally." (Higgins, 1996: 66-67).

Not only are the material conditions for disability created by material progress, advances in social and political technologies also make disability.

The welfare state is one such example of this relationship between advances in technology and the problem of human impairment and limitation. Oliver and Barnes explain that, as we know it now, “disability" can be thought of as a product of public policy (Oliver and Barnes, 2012: 120). In looking through the World Health Organization's reports on Disability which collects data on disability from almost every government in the world, an interesting trend can be observed to prove this contention: in advanced capitalist, "Western," more technology-reliant and technological societies, the disability rate is reported much higher; in countries that are less so, it is generally reported lower. The stark contrast could be made between Australia on the one hand, with a disability incidence rate of close to $19 \%$, and Laos which reports a disability rate of around 1\%(WHO, 2011).

This suggests that how a state measures and accounts for disability varies according to cultural conceptions of what disability is. Since disability is primarily an economic category as far as the modern state is concerned, one could also infer from this data that the organization of the economy in things like the pace of work, and the means of subsistence have an impact on how disability is measured and accounted for. So before the enclosure movement in feudal Britain which threw peasants off their land and effectively robbed them of their means to subsist, disability did not exist as an 
administrative category because people organized in a cooperative, communal mode of subsistence, rather than the competitive, independent wage-labour mode of subsistence which followed and so disenfranchised and impoverished people with impairments. As Oliver and Barnes explain "large groups of dependent people were created by the process of industrialization... Families dependent solely upon the wages of able-bodied individuals could not provide for relatives unable to find work in the new factory system" (Oliver and Barnes, 2012: 83).

This new mode of subsistence that accompanied the mode of production of industrial capitalism worked on the premise that "work in the paid labour market is central to all capitalist societies simply because labour power produces the goods to sustain life. For most people therefore exclusion from or marginalization within the labour market will almost certainly result in poverty, social isolation and alienation" (Oliver and Barnes, 2012: 128). The lives of many people with impairments in early industrial capitalism were thus confined to the margins, to the "poor houses" where the new revolution in technology could not be said to have anything but the most indirect benefit, mixed with considerable detriment.

Here there is an obvious contradiction in the history of technological progress, which in illuminating I hope will remove the need to pursue the relationship between disability and technology in any greater depth here. This contradiction is that the advance of technology, while seeking to free or at least loosen human beings from the bindings of their material condition, has not done so in an even manner. In the following passage, Walter Benjamin notes the folly of conceiving technological progress as a boon to the oppressed:

"Technical developments counted to [the German working class] as the course of the stream, which they thought they were swimming in. From this, it was only a step to the illusion that the factory-labour set forth by the path of technological progress represented a political achievement. [Their movement] defined labour as "the source of all wealth and all culture." Suspecting the worst, Marx responded that human being, who owned no other property aside 
from his labour-power, "must be the slave of other human beings, who... have made themselves into property-owners." Oblivious to this, the confusion only increased, and soon afterwards Josef Dietzgen announced: "Labour is the saviour of modern times... In the... improvement... of labour... consists the wealth, which can now finally fulfil what no redeemer could hitherto achieve."

The messianic pretensions of technological history are here put quite clearly on display. Benjamin continues:

"This vulgar-Marxist concept of what labour is, does not bother to ask the question of how its products affect workers, so long as these are no longer at their disposal. It wishes to perceive only the progression of the exploitation of nature, not the regression of society. ...Labour, as it is henceforth conceived, is tantamount to the exploitation of nature, which is contrasted to the exploitation of the proletariat with naïve self-satisfaction" (Benjamin, 1940: xi).

As Benjamin points out, as far as the oppressed are concerned, it is only in a misguided sense that they share in the benefits of wealth and technical capacity.

In advocating a struggle for the control of the means of production by workers, Marx was more or less advocating for control of technology which could then serve the interests of all instead of the interests of a few. The lesson learned here is that just because the technology exists, does not mean that everyone who could benefit from it has access to control it. Furthermore, in a capitalist system, that access to control is the most jealously guarded thing - access to technology both creates wealth, and requires wealth in the first place. Technology, where it can be enclosed and monopolized, is the domain of capital: if it were shared freely and evenly, it simply would not be capitalism.

To further clarify, technology is indeed important for understanding disability, but in the context of capitalism, it is the distribution and access to this technology which is of the most immediate and real consequence to disability. In saying that technology is subordinate to social relations, and controlled by power, we can move forward to talk about those social relations and manners of organization that facilitate the conditions for access to technology, and not put technology on more of a 
pedestal than it deserves. What must be talked about instead is the creation of scarcity and its political function of restricting access.

\section{4: Scarcity's Utopia}

The foundation for Capitalism, far from being a natural evolutionary stage, is based on primitive accumulation. Primitive accumulation is the process of divorcing the producer from the means of production, which in real terms is the dispossession of people of the means that they rely upon for their very subsistence (Marx, 2011: 188). There is no natural tendency toward this arrangement, or toward greater productivity, this is the result of the capitalist/landlord/free labour class relationship which is a particular, rather than universal, political arrangement (Brenner, 1977). The exclusion, marginalization and dependency of people with disabilities in capitalist societies is therefore as "natural" as the thing that produced it: the emergence of the competitive wage-labour market which is to say it is not natural at all. David McNally explains that

"The simple fact is that across different societies people have always resisted this arrangement. Given virtually any other semi-viable option, people overwhelmingly prefer to cling to precarious conditions as farmers, fishers, hunters and the like rather than sell their human capacities to a buyer. It is only when there is no other way to survive, -- when, in short all other economic options have been taken away from them - that people reluctantly accept a life as wage-labourers" (McNally, 2007: 90-91).

When subsistence is completely controlled by that labour market, it is the people who cannot compete in the labour market who lose the ability to subsist: namely, the disabled.

The marginalization of disabled people is much less a fact of nature in human ecology as it is an 
historical condition created by political economic interests. These interests lay in the rise of private property and the movement to enclose all common lands, and these interests were, in no small part, influenced and directed by the philosophy of classical political economy. These interests were refracted in polemics against the English Poor Laws, which in the late $18^{\text {th }}$ and early $19^{\text {th }}$ century had been the nascent welfare state which took it upon itself to ameliorate the new forms of poverty that had emerged. Karl Polanyi explains the origin of the vision of nature adopted by the Classical Political Economists:

Townsend's Dissertation [on the poor laws], ten years after [Adam Smith's Wealth of Nations], centred on the theorem of the goats and the dogs. The scene is Robinson Crusoe's island in the Pacific Ocean, off the coast of Chile. On this island Juan Fernandez landed a few goats to provide meat in case of future visits. The goats had multiplied at a Biblical rate and became a convenient store of food for the privateers, mostly English, who were molesting Spanish trade. In order to destroy them, the Spanish authorities landed a dog and a bitch, which also, in the course of time, greatly multiplied, and diminished the number of goats on which they fed. "Then a new kind of balance was restored," wrote Townsend. "The weakest of both species were among the first to pay the debt of nature; the most active and vigorous preserved their lives." To which he added: "It is the quantity of food which regulates the number of the human species."(Polanyi, 1948: 114).

This philosophy of nature drops man, the independent wage-labourer who is concerned solely with keeping his own belly full, alone into nature as a predator. That the weak would die or be culled from the herd was not an idea of Charles Darwin and evolutionary biology first: it was articulated by Joseph Townsend, and later Thomas Malthus, one of the founding figures of Classical Political Economy (Young, 1973: 6). Karl Polanyi continues in saying:

Yet neither Darwin's theory of natural selection, nor Malthus' population laws might have exerted any appreciable influence on modern society but for the following maxims which Townsend deduced from his goats and dogs and wished to have applied to the reform of the Poor Law: "Hunger will tame the fiercest animals, it will teach decency and civility, obedience and subjection, to the most perverse. In general it is only hunger which can spur and goad them [the poor] on to labour; yet our laws have said they shall never hunger. The laws, it must be confessed, have likewise said, they shall be compelled to work."' (ibid.)

In suggesting that there is a surplus population that could not survive in harmony with the laws of nature, one should read Townsend's vision of nature as structuring his utopian vision. For what the 
vision of Townsend, Malthus, and many others like them offers is a concept of nature upon which to predicate an ideal society. What is being offered here as nature fundamentally structures the possibilities for what is being offered in utopia; the guiding laws of material possibility fully constrain the political and economic options of the present. This corresponds also with the character of immediate political goals, which for Malthus amounted to a tighter control of the poor and of labour. "The apparently insoluble problem of pauperism was forcing Malthus and Ricardo to endorse Townsend's lapse into naturalism"(ibid: 115), and those whom were hardest hit by this shift in philosophical landscape were those whom had been disabled by the conditions of the labour market.

To talk about what political economists thought, one must also have some sense of what political economy was, or, as Blaney and Inayatullah suggest, one must view it as a cultural project. Political economy grew out of statistics, which was one of the first applications of scientific method to governance. Statistics, through the census was essential to the development of the discipline of political economy during the Enlightenment. Through this pursuit there emerged the very concept of population itself as a measurable thing with testable characteristics (Polanyi 1948: Ch 10). The very concept of the norm, or normality came from statisticians, and significantly enough, virtually all early statisticians were also eugenicists (Davis, 2013: 3). The concept of normality "was implicitly defined as that which advanced progress (or at least that which did not impede it). Abnormality, conversely, was that which pulled humanity back toward its past, toward its animal origins" (Baynton, 2013: 19).

Looking back at a time before the Industrial Revolution, before the rise of Political Economy, and before the concept of "normality," may allow for a clearer picture of the "animal origins" upon which the new utopian vision was founded. In turning to Marshall Sahlins' work on hunter-gatherer societies, one must be brutally honest about the terms upon which actual hunter-gatherering societies exist if we hope to achieve this clarity. 
"The terms are, cold-bloodedly: diminishing returns at the margin of portability, minimum necessary equipment, elimination of duplicates, and so forth - that is to say, infanticide, senilicide, sexual continence for the duration of the nursing period, etc., practices for which many food-collecting peoples are well-known. The presumption that such devices are due to an inability to support more people is probably true - if "support" is understood in the sense of carrying them rather than feeding them. The people eliminated, as hunters sometimes sadly tell, are precisely those who cannot effectively transport themselves, who would hinder movement of family and camp" (Sahlins, 1972: 34).

It is clear that a mobility impairment would not do in the kind of nomadic hunter-gatherer group described above. But the important distinction to make is that this is a decision made based on immediate material limitations which are present and clear, and not based on political priorities.

Sahlins noted that the consistent rule of hunter-gatherers was that no one would starve unless everyone was starving (ibid). The necessity to be mobile is based on real scarcity, whereas in capitalism scarcity is institutionalized though nothing makes it strictly necessary. As Karl Polanyi described, our "animal dependence upon food has been bared and the naked fear of starvation permitted to run loose. Our humiliating enslavement to the material, which all human culture is designed to mitigate, was deliberately made more rigorous" (Polanyi, 1947: 115).

What can be learned from the hunter gatherers is that "our problems are not theirs ... Rather, a pristine affluence colours their economic arrangements, a trust in the abundance of nature's resources rather than despair at their inadequacy of human means" (Sahlins 1972, 29). In comparing industrial society to the Paleolithic, the intent is not to gain policy insights from history, but to put notions of human nature to the test. Though hunter-gatherer society is obviously not the utopia for the disabled, the abundance of means to subsist even relative to capitalism dethrones the edict that scarcity is a constant and universal condition of nature.

Sahlins makes a clear distinction in stating "scarcity is not an intrinsic property of technical means. It is a relation between means and ends"(ibid: 5). In other words, at the baseline of human 
economic organization, scarcity is not about ability, it is about willingness to share. Sahlins goes on to say that "this is the era of hunger unprecedented. Now, in the time of the greatest technical power, is hunger an institution" (ibid: 36).

The lesson here is once again that social orders and the relation of the whole to the means of subsistence is the intervening variable, and not the technological means itself. Though disability and poverty are highly correlated, it must be stressed that they are not the same thing (Bickenbach, 1993), but the fact remains that due to this correlation, scarcity's most severe consequences often fall on the disabled. The problem of "the savage" was not a greedy or capricious nature which led to accumulation and scarcity - the ethnographic records tells exactly the opposite.

Rather, the utopian vision offered by capitalist political economy stresses work as a way to escape the savage, animal state of scarcity which it has imagined. Again, this has more to do with the problems of modernity than those of the pre-industrial world. In this modern, industrial market economy, "where production and distribution are arranged through the behaviour of prices, and all livelihoods depend on getting and spending, insufficiency of material means becomes the explicit, calculable starting point of all economic activity" (Sahlins, 1972: 4). In understanding that this social order is not predicated upon laws of nature, one can also understand the twist in rhetoric at play when "having equipped the hunter with bourgeois impulses and Paleolithic tools, we judge his situation hopeless in advance" (ibid).

The "savage condition" of the hunter-gatherer is imagined as one wherein she is condemned to struggle, starvation and poverty against insufficient means, though this is in reality not the case. The fall from Eden of the hunter-gatherer overlaps with the situation of the disabled in capitalism, who is likewise condemned to struggle, starvation and poverty against the pressures of their own insufficient means. Both the savage and the disabled, in this utopian narrative, are crippled by nature. 


\subsection{Scarcity and the Theory of Impairment}

Scarcity is a political category that does, undeniably, exist as a real phenomenon, but is more often invoked for political purposes. Blaney and Inayatullah explain the logic at work here:

"And yet, there is a trick here. We can name it: it's the immaculate appearance of scarcity abstracted from any specific time/place/culture. We can see this trick in our everyday lives. When I say "no, thank you" to a request by a friend, I couch it in the following terms: "I would like to do what you ask, but I don't have the time to do it." Here I am employing scarcity; not enough hours in the day and all that... But, to say that I wish I had more time to do the things I wanted, or to say that I don't have time to do $\mathrm{x}, \mathrm{y}$ or $\mathrm{z}$, is to also say that $\mathrm{x}, \mathrm{y}$ and $\mathrm{z}$ are not particularly high on my list of priorities at this time, in this place, in this cultural context.’(Blaney and Inayatullah, 2010: 201).

Within the cultural context of capitalism, submitting to the worker-capitalist relationship was the only real way out of the deadlock of scarcity. The other way to subsist was charity, which involved an even greater degree of marginalization to which many people with impairments had to submit.

The welfare state represented a step in a different direction, away from using charity as a basis for addressing need when work already could not. It was an effort toward providing for need and vulnerability in a way that could be deserved by its recipients, and not just gratefully and meekly received as a grace. The "deserving poor" (more or less the equivalent of disabled) were always privileged above "the undeserving poor" (the people "able" to work). But in reality, the deserving poor were an uncomfortable reminder of the wound of wealth, which progress had been unable to mend.

There were a few suggestions how to deal with the condition of the most vulnerable people in industrial society where scarcity had been institutionalized. The eugenicists offered the vision of an able-bodied utopia achieved through what is tantamount to murder; the more charitable advocates of the welfare state offered "poor houses" and later, the total institution of the asylum. The 
institutionalization of "defective" persons during the $19^{\text {th }}$ century in the form of the clinic, the penitentiary and the asylum created sets of institutional knowledges which were hailed as the rational course for dealing with such people (Foucault, 1965).

The institutionalization of both scarcity, and concomitantly disability, depended on establishing an economy of time; the move from task-oriented work to time-oriented work in the Industrial Revolution achieved the fungibility of time and money (Thompson, 2009). This interchangeability set a pace to work, life and subsistence which literally bought all the time and energy of the workers, who in return were given a meagre subsistence. The time for social reproduction, for care of the self and the other, vanished. Not only were people with disabilities categorically unable to produce, they were consumers of the time of people who desperately needed to be working to support themselves. In order to be cared for, then, someone had to be paid to do it. The lack of care for the vulnerable was a product of the newly created scarcity of time for the wage labouring classes.

These developments in the political economy created a culture which espoused something which could be called, in its ideal form, the eugenics of productivity. The increasing rationalization of disability which accompanied the development of capitalism led to the view of impairment as a biological deficiency to be medically treated. It was this culture of trumpeting efficiency and productivity as the highest human virtues that disability became a source of shame which necessitated its physical segregation in institutions (Oliver and Barnes, 2012). The rationalized response to disability was then, simple repression. Disability had no place in the capitalist utopia; it was an uncomfortable reminder of the savage condition in nature, which was antithetical to progress.

To summarize, the position of the disabled was "[derived] from the economic relations of production, the cultural oppression of disabled people associated with the position of the 'flawed body' 
in western culture, and from anxieties of the able-bodied about their own position in the labour market" (Fulcher, 1996: 177, paraphrasing Hevey, 1993). The economic, cultural, and political position of people with disabilities is then perhaps more about able-bodiedness than the material basis of impairment. What is cast as the real and material in disability, namely impairment, is always-already socialized as disability in any actually existing relationship. However, the material aspect of impairment, and its intrinsic limitation, is based on a utopian conception of the civilized human being's relationship with a primordial savage social order in nature, which is competitive, individual and under a condition of universal scarcity.

This "savage condition," as economic anthropology has shown, does not reflect the lived experience of human beings actually in those conditions; scarcity and competition between individuals are absent in hunter-gatherer societies. The demystification of this state of nature would indicate that this relation to nature is not universal, but is always-already a reflection of situation of human beings in capitalism: a work in historical fiction which says more about its author's time than the one being written about. When impairment is invoked as the fact of nature behind the appearance of disability, this simply means that disability is still being treated as a category within in a particular mythology.

Disability designates a political relationship to the mode of production in one's society; and a relationship that is actively nominated as an inability to compete. This political designation is written into raison d'etre of the welfare state, and the medical profession has historically been tasked with making the distinction between disabled and able-bodied.

If impairment is an abstract universal, what designates impairment over non-impairment is not clear. Because impairment is a continuum of many different forms and functions, and without a framework for which of these forms and functions are valuable, there is no intrinsic means by which we can distinguish and categorize this. This distinction is always a political one because it nominates 
members of the social group upon whom scarcity will be invoked. What is left for establishing a threshold of impaired versus non-impaired is a speculation on the probability of survival of certain individuals with certain impairments in particular hypothetical situations. But this exercise in itself would necessarily make survival an apolitical and ahistorical affair of individual concern.

The way out of this is not a social theory of impairment. In making a social theory of impairment, and removing impairment from its place as an abstract universal to examine it as a concrete lived reality, one simply ends up with a carbon copy of what is already known and theorized as disability. Establishing a difference between a social model of impairment and a social model of disability would be an expert-level exercise in hair-splitting. Impairment then, can be more productively thought of as a category of fantasy emerging from a particular concept of the human being's relation to nature.

When pressed into service as a fantasy, impairment reveals itself as a dialectic, as something fundamentally in tension with its own premise. The basis of the dialectic is this: human beings are essentially alienated from nature by their own definition, and furthermore, there is no going back to nature. The cut has already been made. The basis of the dialectic is that it is already broken. Impairment is a horizon, it is a potentiality: it does not yet exist. Impairment is a hypothetical relationship with nature that is always already defined by scarcity, competition and inaccessibility. As such, impairment tries to refuse the proper starting point that it is already broken to replace it with the fantasy that some are not yet imperfect, that there are fully able-bodied independent individuals. This is a crucial line of distinction which the ideology of impairment blurs. Where the ideology of impairment smudges our lens is in propagating the conviction that impairment does exist and is concrete.

"In an ideological edifice, you need some pseudo-concrete image like this to fixate your imagination, and then this image can mobilize us," (Zizek, 2013: unpaginated) and in Ableism, this 
pseudo-concrete image is impairment. Furthermore, when we consider "ideology as a kind of filter: a frame so that if you look at the same ordinary reality through that frame, everything changes. ... It is not that the frame actually adds anything, it is just that the frame opens the abyss of suspicion" (ibid); the suspicion in Ableism is that a disabled existence is a less valuable way of being in the world, and that vulnerability should be repressed.

We would be duped if we were to believe that impairment is a neutral material fact and not the fantasy that stages the tragedy of the able-bodied fall from grace. Impairment, in this precise sense, is the final frontier of nature in our civilized bodies, and the way in which human beings can never escape the horizon of the natural world; after trying to separate humanity from nature, nature returns as impairment to show humanity that it cannot escape. It is a sad and narcissistic scene of those deemed able-bodied staging their own fall from the grace of an Edenic state over and over again. Impairment is the invocation of that fall, and it is ultimately as untenable as the separation between human beings and nature.

The potential for emancipation lies in accepting that "it is already broken," and that there is nothing further behind the appearance of disability linking it to an a-political hierarchical order. There is no fall from grace, there is only the inevitable fact of human vulnerability and interdependence, and the political question of how must these be addressed. 


\section{Chapter 8. CONCLUSION}

Attendant Services is a space where meanings around disability can be remade outside of this fantasy of impairment, through engagement with and care for the other. The realization of interdependence and the culture of social equality, set against this history, are a radical revision of so called "natural" orders and hierarchies. If the social order of Attendant Services is artificial, so is the state of nature whose order it violates. Impairment can no longer function as a fantasy of so called ablebodiedness if impairment is not intrinsically bad. The culture of Attendant Services that allows for people with disabilities to frame their conditions in a positive way resists this fantasy of impairment. This resistance also occurs, in no small part because there is space for the able-bodied to also confront and reject this fantasy. "Disabled" still endures as a category while impairment does not, because disability rests on a political designation whose meaning is fluid, while impairment fixes itself to a positive definition whose meaning is fixed, and fixed to a particular fantasy of Ableism.

Following Davis (2013), who argues that in reality "form follows dysfunction," disability is already a better representation of "the condition itself" than impairment. There is no need for a social theory of impairment, because it already exists: it is called Ableism. As a "natural theory," impairment already rests upon a social theory which simply has the pretensions to call itself "natural." We should wonder about the sources of this "nature," because "What is often being argued, it seems to me, in the 
idea of nature is the idea of man; and this not only generally, or in ultimate ways, but the idea of man in society, indeed the idea of kinds of societies" (R. Williams, 1980: 70-71). We currently live in a society where impairment is seen as bad, as intrinsically limiting, and so forth. But we do not have to accept this society as the ultimate, unchangeable and natural order of the world because frankly, it does not have to be.

The present is made of the memories and possibilities: there is the memory of the past, always filtered through human minds as history; and there is the possibility of the future, always embroiled in the political. History is the constitution of the present, it is the story of this moment which leads to the next. In deciding what the next moment will be, human beings resort to politics: an argument over history. To surrender political will is to let go of the horizon of possibility, to accept one's role as the supporting cast(e) in history.

Survival is a social task, and subsistence is a necessarily an interdependent endeavour. The relationship of the client and the attendant shows how these two actors together perform disability as a social phenomena, and how a different script can offer the opportunity to break the categories and change the performance itself. In following Shakespeare's contention that the role of the able-bodied, as well as the role of care must be examined for the role they play in making disability, I have tested and rejected the other half of his hypothesis, namely toward a social theory of impairment. Tom Shakespeare's push towards a social theory of impairment is resigned to this: that impairment actually is a social phenomenon, but it is inherently negative, therefore any question of why it is inherently negative is a moot point, because it always already just is. It would appear that he is making an argument about nature and/or human nature: positing an unalterable law.

As Blaney and Inayatulah note, the "savage" can never be fully excised from the modern condition; it is an alternative way of being which is constitutive of "modern" forms of social 
organization and being (Blaney and Inayatullah, 2010: 16-18). Attendant Services offers a thoroughly "modern" approach to addressing human limitation through professionalization, commodification, policy and codes of conduct, and a structured work environment. But all of these apparently modern, universalistic and individualized things rely on some fundamentally dismodern ${ }^{12}$ elements to prop them up and give them their substance. The elements of care, contextual morality, friendship, and a cooperative engagement with work exist in tension with their sanitized and institutionalized counterparts.

The attendant is at the heart of the struggle to dress this "wound of wealth" -- the exclusion of people with disabilities -- through a commodified relationship. This attempt is always already incapable to close the wound because commodity relations alienate rather than promote the mutual engagement which care demands. As such, the attendant exists in the centre of this dialectic tension between sanitized progress of commodified relationships versus the gritty, dirty work of social reproduction, upon the exploitation of which the entire civilized edifice rests. The attendant performs a professionalized role based on universal morals, but in order to carry out the actual task of care, both the professionalized (in the sense of detached) and universal (in the sense of universally applicable) elements are subverted by contextual engagement.

Care is repressed as a marginal aspect of the modern economy, but its role is essential in the "social reproduction" -- the basic needs of subsistence - of every economy, no matter how archaic or remote. Claude Meillassoux, an economic anthropologist conducting research on the subsistence economies of primitive agricultural societies, found that the consequence of agricultural cooperation was that "everyone's unspecified labour was mingled in a common product. Everyone's labour is blended and each participates in the product of the other's labour" (Meillassoux, 1978: 292). The

\footnotetext{
${ }^{12}$ To once again follow Lennard Davis, (2013) and borrow a term.
} 
depiction of how resources and labour are distributed presents an economy that is premised upon an ethic of care rather than an ethic of work. That is to say, survival is not the task of the individual worker, but of the communal whole, the main concern of which is caring for its members' needs. Social reproduction, rather than production is foregrounded in this web of interdependent relationships, and in this precise way, it shows a parallel with the attendant-client relationship.

The "end" of this economic relationship is ostensibly the wage of the attendant on one hand and the functional independence of the client on the other. The "means" of production here is care, which as defined by Tronto (1993) is an attentive, responsive, competent and responsible engagement with the other. The means and ends cannot be separated here because "care" is in itself both the means and the end: the attendant must "care" to earn a wage and therefore subsist, and the client requires care to exercise functional independence and therefore subsist. Though both parties participate differently in this process, their labour is nonetheless "mingled in a common product."

The role of the attendant in the liberal moral-political universe is to sanitize and demystify the disabled other: sanitizing occurs both in a crude literal sense of helping with the daily tasks of hygiene as well as figuratively making disability palatable to society by "servicing" it. The attendant demystifies disability by engaging with it on the social and physical level in order to integrate it into the liberal-multicultural social order. Demystifying is a process of stripping away the apparent essence of disability by providing functionality, and in this sense the other is no longer genuinely other, thus can be integrated in a sanitized, non-threatening form into liberal multiculturalism. The liberal ideal of the role of the attendant, however, is not to create a disabled identity, but precisely to prevent an overidentification by society and persons with disabilities themselves with the very idea of disablement.

If the previous paragraph sounded dehumanizing it was to demonstrate the character of the liberal project as detached, sanitary and impersonal. These are not intrinsically bad characteristics, but 
they elide the real role of the attendant and the real work of care, and are hopelessly impotent when it comes to dealing with a particular emergency situation. Care and disability subvert the cultural and humanitarian project of liberal capitalism by the impossibility of their integration into the market-based framework of governance. The ideal of the laissez-faire market is, at its most fundamental, a passive reliance on a natural order of things; care, on the other hand, is unceremoniously thrown into the world of social meaning, where rational passivity is hardly a neutral or natural stance, or even a viable option.

Disability is a state of exception within a market economy, as such it is unlikely that the Attendant Services Program would have existed if it were left to market forces - the category of disability demands an active intervention of care into "the natural order of things" to preserve the humanitarian values of liberalism. It is important here to remember that Attendant Services also represents a very privileged segment of the population of people with disabilities. While the market may work to provide services for a few very privileged people with disabilities, most others will be excluded by that same mechanism, and care-workers will likely be further marginalized in the process.

Care is instrumental in arriving at a concept of history that, as Walter Benjamin suggests, understands that the emergency situation in which we live is the rule, because care addresses the consequences of this situation head on. The concept of impairment is an attempt to bypass this reality and live in the fantasy that some of us are unimpaired. In this precise sense, impairment is an imagined relationship with nature, consistent with liberal economic thought whose intellectual edifice rests on the separation of culture from nature. Disability is a bridge between nature and culture, and care is the way in which human beings traverse that bridge, so to speak; our real and universal material limitation is always a going social concern which has historically demanded the intervention of care.

Impairment is not a representation of this relationship to our "emergency situation." Impairment cannot be passively deferred to as the hard fact of material reality which actually exists beyond the 
social construction of disability. That impairment is a fantasy does not mean that it does not have consequence: precisely the opposite, it is what structures the apolitical reading of disability as personal tragedy. We are kept from understanding the emergency of our situation, which is of our mortality, limitation and finitude, by this fantasy that impairment can be abstract and isolated.

What is greater than the physical fear embodied in the fantasy of impairment -- and this is why the fantasy focuses explicitly on this physical fear-- is the fundamental anxiety that is revealed when, if the subject with the able-bodied fantasy of impairment becomes disabled, it becomes known to the subject that they, as formerly able-bodied were complicit in creating the actual conditions in which they find themselves when disabled. In other words, the social construction of disability opens up a space of too much radical contingency, where negativity (the unknowable consequences of what could have been) and nothingness (the meaninglessness of what is) become too traumatic to confront directly. Impairment fills in the void of utter contingency and negativity with simple meanings of natural order, which brutal as they may be, pose no threat to the status quo.

Disability implicates everyone in its creation, while impairment frees the able-bodied from identifying or over-identifying with their role in making disability. The problem of over-identification with the other is put brilliantly on display in one of the final scenes of Dostoyevsky's The Brothers Karamazov (Book XII, Chapter X). The defence lawyer gives an impassioned speech which implicates the whole cast of the novel and Russian society at large in Dmitry Karamazov's murder of his father. The entire courtroom is moved to tears by this recognition and are deeply struck by the power of this truth. Nonetheless Dmitry is convicted, because this identification with the other is, in this moment, a tremendous threat to the authority and universal morality of the legal system. The argument of the defence lawyer is not an argument positing what is and what should be, but rather one about the tension between what already is, yet cannot be within the existing framework of what should be. 
The modern condition of disability is something that for liberal capitalism both is and cannot be. The horizon of technological progress is looked upon to erase this tension. Impairment, as part of the able-bodied fantasy, clings to an able-bodiedness which is temporary and always incomplete, and suggests a fundamentally normative position that people should not be impaired.

In order to have an emancipatory disability politics we must forego the crutch of impairment that allows us to distance ourselves from the reality of disability. The culture of Attendant Services at Carleton University and Algonquin College represents an achievement in a genuine engagement on more or less equal terms between care and disability, the non-bodied and disabled. Both clients and attendants at this program share the material and social consequences of disability and perform the task of care mutually. The radically open engagement of the peer-to-peer care relationship makes a reevaluation of ableist norms and ideals possible. The result is that care and disability both recede into the background of these interactions. Disability does not define the clients, and the attendant is not rendered a mere instrument of self-help.

Despite its flaws, Attendant Services is an oasis; there may be change and humanity within the program, but the attendants and clients both are left on the margins of political and economic priorities in the greater scheme of things. The model of this program nonetheless presents the opportunity for these experiences and this culture to reshape disability on a much larger level. Programs like this are a major step beyond accessibility and toward equality. This equality is not simply achieved by including people with disabilities, but by transforming both the disabled and the non-disabled through an engagement with the everyday tasks of care. 


\section{REFERENCES}

Abberly, Paul. "Work, Impairment and Utopia." in Disability and Society: Emerging Issues and Insights. Ed. Len Barton. Addison Wesley Longman Ltd, Essex: 1996.

Albrecht, Gary. The Disability Business: Rehabilitation in America. Sage Publications, London: 1992.

Armstrong, Pat. "Restructuring the Public and Private, Women's Paid and Unpaid Work." in Challenging the Public/Private Divide: Feminism, Law, and Public Policy. University of Toronto Press, Toronto, 1997.

Baynton, Douglas. "Disability and the Justification of Inequality in American History" in The Disability Studies Reader, $4^{\text {th }}$ edition. Ed. Lennard Davis. Routledge, New York: 2013.

Benjamin, Walter. "On the Concept of History” 1940. http://www.marxists.org/reference/archive/benjamin/1940/history.htm

Benton, Ted. "Marx and Natural Limits” in New Left Review, Vol. 178, London: 1989.

Bickenbach, Jerome E. Physical Disability and Social Policy. University of Toronto Press, Toronto: 1993.

Blaney, David and Naeem Inayatullah. Savage Economics: Wealth Poverty, and the Temporal Walls of Capitalism. New York: Routledge, 2010.

Braun, Bruce. The Intemperate Rainforest: Nature, Culture, and Power on Canada's West Coast. University of Minnesota Press, Minneapolis: 2002.

Braun, Bruce and Noel Castree, eds. Social Nature: Theory Practice and Politics. Blackwell, Oxford: 2001 .

Braun, Bruce and Jan Moeckli, "Gendered Natures" in Social Nature: Theory Practice and Politics. eds. Bruce Braun and Noel Castree, Blackwell, Oxford: 2001.

Brewer, D. J. Opinion of the Kansas Supreme Court. Griffith v. Osawkee Township. 14 Kansas 418. 
Reprinted in Abbot 1940. 1875.

COPOH. Defining the Parameters of Independent Living. ed. April D'Aubin. Winnipeg, Manitoba, Year not given.

Castree, Noel. "Socializing Nature," in Social Nature: Theory Practice and Politics. eds. Bruce Braun and Noel Castree, Blackwell, Oxford: 2001.

Charlton, James. Nothing About Us Without Us: Disability Oppression and Empowerment. University of California Press, Berkeley: 1998

Clow, Michael. "Bahro on Ecology" in Studies in Political Economy, Vol. 20. Ottawa: 1986.

Cronon, William. Uncommon Ground: Rethinking the Human Place in Nature. W W Norton \& Company Incorporated. 1995.

http://www.williamcronon.net/writing/Trouble_with_Wilderness_Main.html

Daly, Herman. Beyond Growth. Beacon Press, Boston MA, 1996.

Davis, Lennard. "Disability, Normality and Power." in The Disability Studies Reader, $4^{\text {th }}$ edition. Ed. Lennard Davis. Routledge, New York: 2013.

Davis, Lennard. "The End of Identity Politics: On Disability as an Unstable Category." in The Disability Studies Reader, $4^{\text {th }}$ edition. Ed. Lennard Davis. Routledge, New York: 2013.

DeJong, Gerben. "Independent Living: From Social Movement to Analytic Paradigm." in Arch Phys Med Rehabil, Vol 60, 1979.

DFID, "Disability, poverty and development" 2000, 2011. dfid.gov.uk/pubs

Dostoyevsky, Fyodor. The Brothers Karamazov. Orig. 1880. http://www.gutenberg.org/ebooks/28054

Emerson, Robert, Rachel Fretz and Linda Shaw. Writing Ethnographic Fieldnotes, Chicago: University of Chicago Press, 2011.

Finklestein, Vic. "A Question of Choice.” in Disability Challenge. No. 1, UPIAS, 1981. http://disability-studies.leeds.ac.uk/files/library/finkelstein-A-Question-of-Choice.pdf

Foucault, M., Madness and Civilization: A History of Insanity in the Age of Reason. New York: Pantheon, 1965.

Fulcher, Gillian. "Beyond Normalization but not Utopia" in Disability and Society: Emerging Issues and Insights. Ed. Len Barton. Addison Wesley Longman Ltd, Essex, 1996.

Gluckman, Max. "Anthropological Problems Arising from the African Industrial Revolution." in Industrial Work and Life, An Anthropological Reader eds. Masimiliano Mollona, Geert de Neve, 
and Johnathan Parry. Berg, New York, 2009.

Godelier, Maurice. "The Object and Method of Economic Anthropology." in Relations of Production: Marxist Approaches to Economic Anthropology, ed. David Seddon. Frank Cass and Company Limited, London, 1978.

Goffman, Erving. "On the Characteristics of Total Institutions" in SAGE Encyclopedia of Social Theory, London: Sage Publications, Inc. 2013. Originally published in Asylum, 1961.

Goodin, Robert E. Protecting the Vulnerable: A Reanalysis of our Social Responsibilities. Chicago: University of Chicago Press, 1985.

Hann, Chris and Keith Hart. Economic Anthropology: History, Ethnography, Critique. Cambridge: Polity Press, 2011.

Higgins, Paul C. Making Disability: Exploring the Social Transformation of Human Variation. Charles C. Thomas Publisher, Springfield Illinois, 1992.

Hobbes, Thomas. Leviathan: Or the Matter, Form and Power of a Commonwealth, Ecclesiastical and Civil. London: J.M. Dent and Sons LTD, 1965, orig. 1651.

Lord, John. Impact: Changing the Way We View Disability: The History, Perspective, and Vision of the Independent Living Movement in Canada. Ottawa, Independent Living Canada, 2010.

Marx, Karl. Capital: Volume One: A Critique of Political Economy. Ed. Friedrich Engels, translated by Samuel Moore and Edward Aveling, Dover Edition, New York: Dover Publishing Inc., 2011, orig. 1906.

Matza, David. Becoming Deviant. New Jersey: Prentice-Hall, Inc. 1969.

McNally, David. Another World is Possible: Globalization and Anti-Capitalism. The Merlin Press, Monmouth, Wales 2006.

McRuer, Robert. "Compulsory Able-Bodiedness and Queer/Disabled Experience." in The Disability Studies Reader, $4^{\text {th }}$ edition. Ed. Lennard Davis. Routledge, New York: 2013.

Meillassoux, Claude. "Kinship Relations and Relations of Production" in Relations of Production: Marxist Approaches to Economic Anthropology, ed. David Seddon. Frank Cass and Company Limited, London, 1978. (a)

Meillassoux, Claude. "'The Economy' in Agricultural Self-Sustaining Societies: A Preliminary Analysis" in Relations of Production: Marxist Approaches to Economic Anthropology, ed. David Seddon. Frank Cass and Company Limited, London, 1978. (b)

Morris, Jenny. Independent Lives: Community Care of Disabled People. MacMillan Press, Ltd, London, 
1993.

Oliver, Michael. "A Sociology of Disability or a Disablist Sociology?” in Disability and Society: Emerging Issues and Insights. Ed. Len Barton. Addison Wesley Longman Ltd, Essex, 1996.

Oliver, Michael and Colin Barnes. The New Politics of Disablement. Palgrave MacMillan, Hampshire, UK, 2012 .

Paul Menton Centre For Students with Disabilities "History of the Attendant Services Program" http://carleton.ca/pmc/attendant-services-program/history/

Polanyi, Karl. The Great Transformation: The Political and Economic Origins of our Times. Beacon Press, Boston, 2001. Orig. 1945.

Rein, M. “The Strange Case of Public Dependency,” in Social Policy, New York: Random House, 1965.

Rose, Gillian. "Situating Knowledges: Positionality, Reflexivities, and Other Tactics" in Progressive Human Geography, vol. 21 no. 3, 1997.

Rousseau, J. J. "The Social Contract” orig. 1762, accessed at http://www.constitution.org/jjr/socon.htm, Feb. 13, 2014.

Ryan, J. and F. Thomas. The Politics of Mental Handicap. Penguin, Harmondsworth: 1980.

Sahlins, Marshall. Stone Age Economics. Aldine-Atherton Inc., Chicago, 1972.

Shakespeare, Tom. Disability Rights and Wrongs. Routledge, New York: 2006.

Shakespeare, Tom. “The Social Model of Disability. "in The Disability Studies Reader, $4^{\text {th }}$ edition. Ed. Lennard Davis. Routledge, New York: 2013.

Smith, Neil. Uneven Development: Nature, Capital and the Production of Space. 1984. http://neilsmith.net/unev en-development

Strauss, Anselm and Juliet Corbin. Grounded Theory in Practice. London: SAGE Publications, Inc. 1997.

Taylor, Steven J. and Robert Bogdan. Introduction to Qualitative Research Methods, $3^{\text {rd }}$ Edition. Toronto, John Wiley and Sons Inc., 1998.

Taylor, Steven J. and Robert Bogdan. "The Social Construction of Humanness" in Introduction to Qualitative Research Methods, $3^{\text {rd }}$ Edition. Toronto, John Wiley and Sons Inc., 1998.

Thompson, E.P. "Time, Work Discipline, and Industrial Capitalism.” in Industrial Work and Life, An Anthropological Reader eds. Masimiliano Mollona, Geert de Neve, and Johnathan Parry. Berg, 
New York, 2009.

Tremain, Shelley. "On the Subject of Impairment." in Disability/Postmodernity: Embodying Disability Theory, eds. Tom Shakespeare and M. Corker. Continuum, London: 2002.

Tronto, Joan. Moral Boundaries: A Political Argument for an Ethic of Care. New York: Routledge, 1993.

Tronto, Joan. "Creation Caring Institutions: Politics, Plurality, and Purpose." in Ethics and Social Welfare, 4:2, 158-171, 2010. DOI: 10.1080/17496535.2010.484259

UNENABLE, “Factsheet,” 2011, via http://drpi.research.yorku.ca/

UPIAS, "Fundamental Principles of Disability." Union of the Physically Impaired Against Segregation, London: 1976. http://disability-studies.leeds.ac.uk/files/library/UPIAS-fundamentalprinciples.pdf

World Health Organization. "World Report on Disability: Southeast Asia and Oceania.” 2011.

Wilde, Oscar. "The Soul of Man Under Socialism” http://www.gutenberg.org/ebooks/1017

Williams, Fiona. "In and Beyond New Labour: Towards a New Political Ethics of Care." in Critical Social Policy, SAGE publications, 2001.

Williams, Raymond. "Ideas of Nature," in Problems in Materialism and Culture. Verso Books, London, 1980.

Willis, Paul E. Learning to Labour: How Working Class Kids Get Working Class Jobs. New York: Columbia University Press, 1977.

World Bank, 2008, via http://drpi.research.yorku.ca/

Young, Robert M. “The Human Limits of Nature” orig. 1973, accessed January 152014 at http:/human-nature.com/rmyoung/papers/

Zizek, Slavoj. "Rules, Race and Mel Gibson.” European Grad School Video, 2006 https://www.youtube.com/watch?v=5BIUkUUtvFI

Zizek, Slavoj. The Pervert's Guide to Ideology. Dir. Sophie Fiennes. Ireland: Blinder Films, 2013. www.youtube.com/watch?v=uejTAJ3R3ic 
Sub-Appendix A: Informed Consent Form

Carleton

U N I V E R S I T Y

Canada's Capital University

Consent Form

Title: A Cultural Study of Work in an Independent Living Attendant Service Program

Date of ethics clearance: To be determined by the REB

Ethics Clearance for the Collection of Data Expires: To be determined by the REB

I choose to participate in a study on

Attendant-Client relations in Independent Living situations. This study aims to examine the culture and ideas of work within these arrangements. The researcher for this study is Graham Macdonald in the Institute of Political Economy at Carleton University. He is working under the supervision of Prof. Susan Braedley in the Department of Social Work at Carleton University.

This study involves one 60 minute interview. With your consent, interviews will be audiorecorded. Once the recording has been transcribed, the audio-recording will be destroyed.

As this project will ask you about your employment, there are some potential professional risks to you if your statements are critical of your employer. While this risk is expected to be minimal, I will take precautions to protect your identity. This will be done by keeping all responses anonymous and allowing you to request that certain responses not be included in the final project. Should you experience any distress during the interview, you will be provided with contact information for counseling services available nearby.

You have the right to end your participation in the study at any time, for any reason, up until the end of January 2014. You can withdraw by phoning or emailing the researcher or the research supervisor. If you withdraw from the study, all information you have provided will be immediately destroyed. 
All research data, including audio-recordings and any notes will be encrypted and passwordprotected. Any hard copies of data (including any handwritten notes or USB keys) will be kept in a locked cabinet at Carleton University. Research data will only be accessible by the researcher and the research supervisor.

Once the project is completed, all research data will be kept for five years and potentially used for other research projects on this same topic. At the end of five years, all research data will be securely destroyed. (Electronic data will be erased and hard copies will be shredded.)

If you would like a copy of the finished research project, you are invited to contact the researcher to request an electronic copy which will be provided to you.

This project was reviewed by the Carleton University Research Ethics Board, which provided clearance to carry out the research. Should you have questions or concerns related to your involvement in this research, please contact:

\section{REB contact information:}

Professor Andy Adler, Chair

Professor Louise Heslop, Vice-Chair

Research Ethics Board

Carleton University

1325 Dunton Tower

1125 Colonel By Drive

Ottawa, ON K1S 5B6

Tel: 613-520-2517

ethics@carleton.ca

Researcher contact information:

Graham Macdonald

Institue: Political Economy

Carleton University

Email: Graham.macdonald@carleton.ca
Supervisor contact information:

Prof. Susan Braedley

Department of Social Work

Carleton University

Email: Susan.Braedley@carleton.ca

Do you agree to be audio-recorded: Yes No

Signature of participant

Signature of researcher

\section{Date}

Date 


\section{Sub-Appendix B: Interview Schedules}

\section{Key Informant Semi-structured Interview Guide Attendants and ex-attendants}

This interview will take approximately 45-90 minutes. The following lists of topics will be discussed in the interviews. This is a semi-structured interview schedule therefore the topics will vary slightly based on the answers to questions.

\section{Individual Background:}

1 - How long have you been/did you work at the program? What was your first impression of this job?

2 - How and why did you come to work this job?

\section{General Descriptive:}

3 - What experience or acquaintance did you have with disability or people with disabilities when you began working here?

4 - Did your ideas about disability and people with disabilities change throughout your time as an attendant?

5 - How did/do you see your relationship as an attendant to the client? (as equals, as employer/employee, as care-giver/care-receiver, as friends etc.)

6 - Do you see yourself as different from your clients, why/why not and in what ways? Do you see yourself as different from people with disabilities, in what ways and why/why not?

7 - Do you feel that the clients were ultimately dependent upon you or other attendants for their wellbeing? Did you feel that your well-being was dependent upon them?

8 - In a hypothetical state of nature, do you think your clients would survive, with or without your help?

9 - Do you think disability is a problem? And if so, how is it a problem, and how may it be fixed? 
Are there any questions you feel I should have asked, and didn't?

Thank you for your time and your participation

\section{Key informant Semi-structured Interview Guide Ex-Clients and Clients}

This interview will take approximately 60-90 minutes. The following lists of topics will be discussed in the interviews. This is a semi-structured interview schedule therefore the topics will vary slightly based on the answers to questions.

\section{Individual Background:}

1 - What is the nature of your impairment?

2 - How long have you had attendants? How long have you been in an Independent Living arrangement?

\section{General Descriptive:}

3 - What has your experience been with this program and the attendants, overall?

4 - Do you feel that this program contributed to, or took away from your independence?

5 - How would you characterize your relationship to the attendants? Are they friends, co-workers, employees, extensions of your body?

6 - Do you feel that the attendants are dependent on you in any way?

7 - Do you feel that you are different from your attendants, why/why not and in what ways/how?

8 - In your everyday life, do you find that your impairment gets in the way of what you want to do?

9 - In your everyday life, do you find that the way others treat you has anything to do with your impairment? Does this help or hinder you, in what ways and how?

10 - Which would you say - your impairment or how people treat you because of your impairment - is a bigger obstacle for you?

11 - Do you, or have you ever seen your impairment as a positive thing? 
12 - Do you feel that your impairment is natural?

13 - Do you feel separate from nature because of your impairment?

Are there any questions you feel I should have asked, and didn't?

Thank you for your time and your participation

Sub-Appendix C: Key Informant (Attendants) Interview Recruitment Email or Phone Call

Dear

I am conducting a research project to examine Independent Living arrangements for people with disabilities, and attendant-client relations specifically. I would like to talk with you about this research project, to see if you would agree to be interviewed, at a convenient time. This research will contribute to my thesis project, which I anticipate to lead to academic publications.

I am asking your help with the following:

1. To identify key points of friction and conflict between attendants and clients

2. To identify where policy and practice diverge from one another in Independent Living programs.

3 . To gain a better sense of the actual lived relationships between attendants and clients

4. To gain understanding of attendants' attitudes to their work and their clients

If you are willing to participate, please respond to this email. Upon receiving your agreement, I will contact you by telephone to set up an appointment for the interview. We will also provide you with an information and informed consent form in advance. I look forward to speaking with you! 
Sub-Appendix D: Letter of Introduction

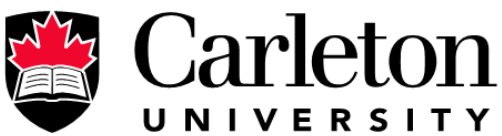

Canada's Capital University

\section{Letter of Invitation}

Title: A Cultural Study of Work in an Independent Living Attendant Service Program

Date of ethics clearance: To be determined by the REB

Ethics Clearance for the Collection of Data Expires: To be determined by the REB

November 27, 2013

Dear Sir or Madam,

My name is Graham Macdonald and I am a Master's student in the Institute of Political Economy at Carleton University. I am working on a research project under the supervision of Prof. Susan Braedley.

I am writing to you today to invite you to participate in a study on AttendantClient relations in Independent Living situations. This study aims to examine the culture and ideas of work within these arrangements.

This study involves one 60 minute interview that will take place in a mutually convenient, safe location. With your consent, interviews will be audio-recorded. Once the recording has been transcribed, the audio-recording will be destroyed. 
While this project does involve some professional and emotional risks, care will be taken to protect your identity. This will be done by keeping all responses anonymous and allowing you to request that certain responses not be included in the final project.

You will have the right to end your participation in the study at any time, for any reason, up until the end of January 2014. If you choose to withdraw, all the information you have provided will be destroyed.

As a token of appreciation, I will be providing you with refreshments during the interview. No other compensation will be provided.

All research data, including audio-recordings and any notes will be encrypted and password-protected. Any hard copies of data (including any handwritten notes or USB keys) will be kept in a locked cabinet at Carleton University. Research data will only be accessible by the researcher and the research supervisor.

This project was reviewed by the Carleton University Research Ethics Board, which provided clearance to carry out the research. Should you have questions or concerns related to your involvement in this research, please contact:

\section{REB contact information:}

Professor Andy Adler, Chair

Professor Louise Heslop, Vice-Chair

Research Ethics Board

Carleton University

1325 Dunton Tower

1125 Colonel By Drive

Ottawa, ON K1S 5B6

Tel: $613-520-2517$

ethics@carleton.ca

If you would like to participate in this research project, or have any questions, please contact me at graham.macdonald@carleton.ca.

Sincerely,

(Insert signature)

Graham G Macdonald 
Sub-Appendix E: Research Ethics Protocol Application

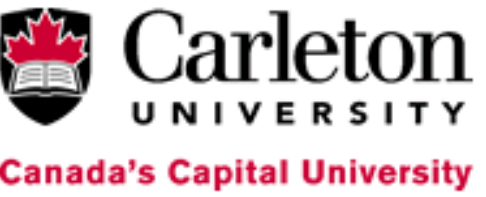

\author{
Research Ethics Board \\ 1325 Dunton Tower \\ Tel: 613-520-2517 \\ ethics@carleton.ca
}

\title{
Research Ethics Protocol Application
}

Office use only:

Project number:

Type of review: $\square$ Expedited $\square$ Full

\section{Section 1: Project Registration}

\subsection{Project Title:}

The Political Ecology of Independence: Ethnography of an Independent Living Program

1.2 Purpose of the research (one or two sentences):

To build theory based on the experiences of attendants and clients in Independent Living programs, in order to address a lack of attention in the literature to actual lived relationships between able-bodied and disabled people.

1.3 Researcher Information: (Students must also complete the supervisor section)

Name: Graham G Macdonald

Department: Institute of Political Economy

University E-mail Address: graham_macdonald@carleton.ca

Position: 
Faculty Member

$\square$ Instructor or Sessional

$\square$ Library staff

Post-Doctoral

Fellow

$\square$ Ph.D. student

x Master's student

$\square 4^{\text {th }}$ year project

Undergraduate

\subsection{Thesis or Project supervisor (Students only)}

Name: Susan Braedley

Department: Social Work

University E-mail Address: susan_braedley@carleton.ca

\subsection{Was this project granted an Approval in Principle for the purpose of releasing research funds? $\square$ Yes $\quad x$ No}

If Yes: Please provide the project number:

1.6 Is this protocol a main or umbrella application for others projects? $\square$ Yes $x$ No

\subsection{Proposed Research Dates:}

Date to begin recruiting participants: October $30^{\text {th }}, 2013$

Date project will conclude: February $28^{\text {th }}, 2014$

1.8 Other researchers: $\mathbf{x}$ Not applicable

\begin{tabular}{|l|l|l|}
\hline Name & E-mail Address & Role \\
\hline & & \\
\hline & & \\
\hline & & \\
\hline & & \\
\hline
\end{tabular}

1.8 Student Research Assistants: x Not applicable $\quad \square$ To be named later

\begin{tabular}{|l|l|l|}
\hline Name & Department/School & E-mail \\
\hline & & \\
\hline & & \\
\hline & & \\
\hline
\end{tabular}

1.9 Research funding: Is this project funded? $\square$ Received $\square$ Pending $\quad \mathrm{x}$ Not funded 
Funding source and program

If funded, will other protocols be submitted under this funding? $\square$ Yes $\quad \square$ No

\subsection{Researcher signature:}

I agree to conduct this research in accordance with the Tri-Council Policy Statement: Ethical Conduct for Research Involving Humans $2^{\text {nd }}$ edition the Carleton University Policies and

Procedures for the Ethical Conduct of Research and the conditions of clearance established by the Carleton University Research Ethics Board.

Signature of researcher

\section{Research supervisor (Student projects only)}

I have assisted with, read and approved the research ethics protocol. I will ensure that the student researcher conducts the research in accordance with the Tri-Council Policy Statement: Ethical Conduct for Research Involving Humans $2^{\text {nd }}$ edition, the Carleton University Policies and Procedures for the Ethical Conduct of Research and the conditions of clearance established by the Carleton University Research Ethics Board and agree to provide all necessary supervision to the student.

Signature

Date:

\section{Section 2: Research Project Information (Complete all sections below)}

\subsection{Description of research project and its objectives: (Limit 200 words.)}

This research project is an interdisciplinary approach to the political economy of disability which aims to put different disciplines and theories in conversation with one another. My case study will be an independent living attendant service program based on Carleton $U$ and Algonquin College campuses. These programs hire students to help students with physical impairments living in residence with physical tasks in their daily lives. These tasks range from getting out of bed, going to the washroom, to helping to cook and eat, and doing laundry.

The projects is intended to fill a gap where attendants perspectives are not represented, and adequate attention is not given to the nuances of the relationships between disabled and able-bodied people. Definitions of disability have polarized between a medical positivist outlook and a socialconstructionist viewpoint, both of which in different ways leave the everyday lives of disabled people and their attendants largley unrepresented (Tom Shakespeare, Disability Rights and Wrongs, 2006). 
This project will take a social-ecology approach to the relationship between impairment (represented by the WHO as the physical fact of loss of function) and disability (the social-environmental consequences of impairment), to expose the limitations of a strong distinction between the two. The impairment/disability distinction plays a key role in casting the "disabled" as oppressed by definition, and the attendant as a tool, a non-entity, or an oppressor. It is the aim of this project to build a theory which can step beyond that distinction, and the everyday lives of physically impaired people and their attendants is its grounding.

2.2 Methodology/Procedures: Describe all methods and procedures that will be used to obtain data

Participant observation: working as an attendant with Attendant Services at Carleton and taking detailed fieldnotes three days a week for two months. This will include the doing the regular duties of an attendant, in a workplace in which I have worked for two years; the only difference will be that I will take fieldnotes on my down-time. My research in this capacity will be unobtrusive and almost invisible to others involved in the program and will not effect the regular functioning of service provision. Due to the nature of the activities which an attendant participates in, I will be careful to not reveal any information that is unnecessarily invasive, in ine with Article 10.3 of the Tri Council statement on Research Ethics (second edition) concerning the privacy of those observed, and will take full consideration of the implications of Article 4.7 which concerns the study of Vulnerable Populations. In Line with article 10.2 of the Tri-Council statement on research ethics (second edition), I will disclose the purposes of my research, with the help of the Director of the Program, through the attendant and client email network before beginning my participant observation.

Semi-structured interviews: I will conduct up to 12 semi structured interviews with former clients of the program and former and current attendants there about their experiences with this particular program. Due to the concerns outlined in Article 4.7 of the Tri-Council statement on Research Ethics, dealing with research involving Vulnerable Populations, I have chosen not to interview clients who are currently living under the service provision of Attendant Services Carleton, because I am focusing on more high-needs clients, the implications of interviewing someone about the service they are currently receiving and will be receiving for the foreseeable future could prove problematic or invasive. Also, I will take special precaution interviewing people about attendant service provision while $I$ am their attendant due to how that may put them in a more vulnerable position. One exception would be clients who will be leaving before this study is completed or published, which would be before May 2014.

In interviewing attendants, the risk is less because they are not in a position of vulnerability, and I will be working only in parallel and not in a direct relationship with them, though I will have a preference for ex-attendants whose testimony may be more open and candid.

The interviewees will be contacted through email, potential candidates will be identified through the contacts I have from working in this community for the past two years.

2.3 Location where the research will take place:

Carleton University X Region of Ottawa-Carleton

Canada (please specify):

Other (please specify):

2.4 Will additional ethics reviews be required? $\square$ Yes $\quad x$ No

If Yes, please provide the name, address and contact information/person for each board or 
committee.

2.5 Visa or research license required:

Yes. Documentation attached

Yes. Documentation to follow

$x$ No

\section{Faculty projects only:}

\subsection{Training and supervision of student researchers: $\quad \square$ Yes $\quad$ o No}

a) Training/experience: Please outline the training that student researchers will receive before commencing work on this project. Include information about their experience to date and their qualifications to work on the project.

b) Supervision: Please outline the supervision plan for student researchers.

\section{Section 3: Research Participants}

\subsection{Recruitment}

a) Describe how potential research participants will be identified and recruited. (Include information on recruitment methods; i.e. email, letter, poster, etc., and where applicable the location of recruitment.)

I will work by word of mouth with contacts I have gained through working with this community for the past two years, to identify potential interviewees. See Appendices 4 and 5 for recruitment emails.

b) Proposed number of participants in the study: 12, plus the workplace itself as a site of participant observation (this would potentially be $30-50$ indirect subjects).

c) Vulnerable research population: $\square$ Not applicable

Describe steps to be taken to ensure that the needs of research participants are respected and the collection of data method will ensure the safe and ethical conduct of the research. Where applicable describe your knowledge, training and experience working with the identified population.

I have worked in this community and this workplace for two years, I will not violate the ethical frameworks of the attendant job in order to do research, or conduct my research in a way counter to the ethical framework of Article 4.7 of the Tri-Council statement on Research Ethics that deals with working with vulnerable populations. The research participants will be interviewed on a voluntary 
basis, and may terminate the interview at any time should they feel justified in doing so. No data, quotes or testimony will be attributed to any individual unless otherwise approved and consented to by that individual. Clients currently in the program and who are not leaving in the near future will not be interviewed.

3.2 Append the required recruitment documents. (Letters, Telephone scripts, emails, posters, etc. Please identify each clearly, example: Appendix 1: Letter of Invitation)

Appendix 1 - Interview Schedule for Attendants/Ex-attendants

Appendix 2 - Interview Schedule for ex-clients

Appendix 3 - Informed Consent interview form

Appendix 4 - Recruitment Email - attendants/ex-attendants

Appendix 5 - Recruitment Email - ex-Clients

\section{Section 4: Conflict of Interest/Power Relationships}

4.1 Are potential research participants employees, clients or persons you have worked with in a professional or volunteer capacity (past or present)? $\times$ Yes $\quad \square$ No

If Yes: Please explain your relationship to or authority over the potential participants and what steps you will take to ensure that the participants' decision to take part in the research will not be influenced by their relationship to you.

I have worked with many of the people I plan to interview (attendants and clients) some are friends, some acquaintances. These friendships and acquaintances have been formed while working at Attendant Services at Carleton University and Algonquin College. I have worked alongside these attendants and for these former clients in the past. I continue to keep in touch with many of them. The interviews will take place on a voluntary basis, and may be terminated at any time if the interviewee feels uncomfortable. There is minimal risk involved to the interviewees, though some subjects may be difficult to talk about for some people. The relationship during the interview will be kept at a professional distance.

\subsection{Are potential research participants, friends, relatives or students? $\times$ Yes}

$\square$ No

If Yes: Please explain your relationship to or authority over the potential participants and what steps you will take to ensure that the participants' decision to take part in the research will not be influenced by their relationship to you.

I have worked with many of the people I plan to interview (attendants and clients) some are friends, some acquaintances. These friendships and acquaintances have been formed while working at Attendant Services at Carleton University and Algonquin College. I have worked alongside these attendants and for these former clients in the past. I continue to keep in touch with many of them. The interviews will take place on a voluntary basis, and may be terminated at any time if the interviewee feels uncomfortable. There is minimal risk involved to the interviewees, though some subjects may be difficult to talk about for some people. The relationship during the interview will be kept at a professional distance. 


\subsection{Do you or any members of the research team have any financial and/or commercialization interest in the research results? $\square$ Yes $x$ No}

If Yes: Please explain in detail your interest.

\section{Section 5: Risks and Benefits}

5.1 Risks to participants: (Check all that apply)

Physical harm or discomfort (This includes any bodily contact, application of equipment, management of any substance.)

x Psychological/emotional harm (This includes feeling demeaned, embarrassed worried or upset.)

Social and economic harm (This includes loss of status, reputation, ridicule form peers, family or friends.)

Economic risk (This includes loss of income, threat to employment, loss of work time)

Legal risk (This includes possible criminal charges or legal suit, duty to report.)

$x$ Other inconveniences (This includes travel costs, disruption to normal activities, time commitment, etc.)

\section{Explain in detail all possible risks to research participants.}

The risks are very minor to participants, the main one being that perhaps some subjects will be emotionally draining to discuss for some individuals, though much of what I hope to enquire about are experiences people encounter on a regular basis, and thus talking about it is not likely to traumatize any interviewee. I do realize that even these experiences, when looked at from a different angle, in a different situation may have an emotional impact. The participant observation poses little risk, given that I am an experienced attendant, and the only difference will be my private recording of fieldnotes, away from clients and unbeknownst to co-workers. The main risk is associated with the privacy and confidentiality of the research participants.

5.2 Managing risk: Describe what steps will be taken to reduce harm to participants.

I will be sensitive in guiding the semi-structured interviews in a way which is not emotionally damaging to those taking place in it, I hope to make it a positive experience for the participants and not a chore or a depressing reflection. In participant observation I will try to maintain the most confidentiality possible and will refrain from using names or specifics that could lead an individual to be identified. I will pay for transport, and food for whomever chooses to participate in an interview.

5.3 Deception: Is there any deception involved in this research project? $\square$ Yes $\quad$ x No

If Yes: Please describe why participants will be deceived, how the deception will be carried out and how and when you will debrief participants. 
5.4 Are there any risks to you or the research team? $\square$ Yes $\quad x$ No

If Yes: Please describe the steps that will be taken to ensure researcher safety.

5.5 Are there any benefits to the research participants? $x$ Yes o No If Yes: Please describe those benefits.

Only very indirect ones. It can be empowering and validating for some to have a platform to tell their stories and experiences and have them reflected upon. If this research is published, it could potentially have a modest impact on policy.

5.6 If you are working with an agency or community group describe what benefits they may receive from this research.

Attendant Services at Carleton may gain some exposure for being the one program of its kind in a Canadian University, there is a small chance they may also have policy applications for some of the findings of the research.

\section{Section 6: Compensation/Remuneration}

\subsection{Will research participants (including an organization) receive compensation or remuneration for their participation? $\quad \square$ Yes $\quad x$ No}

If Yes:

a) Describe the compensation/remuneration (money, gift, transportation, childcare costs, etc.)

b) What is the monetary value of the compensation/remuneration?

\subsection{Will you or members of the research team provide time or assistance to the community participants? $x$ Yes $\quad \square$ No}

If Yes: Please describe the assistance that will be provided.

In the capacity of an attendant, yes. 


\section{Section 7: Anonymity and Confidentiality}

7.1 Anonymity (Treatment of the identity of participants)

a) Will the identity of participants be known to the researcher(s) during the collection of personal information, data gathering or testing?
$x$ Yes
$\square$ No

b) Will the identity of participants be revealed in any reports, papers, research articles, recordings, photographs or presentations, etc?

$\square$ Yes $\quad x$ No

c) Will the identity of participants be known to other participants in the study?

$x$ Yes $\square$ No

d) Will the identity of participants be known to non-participants in the study? (colleagues, family friends of the participants)

$\square$ Yes $\quad x$ No

7.2 Confidentiality: (Attribution of responses and data)

a) The responses/data collected will not be attributed to participants. $x$ Yes o No

b) The data collected will be attributed to participants

$\square$ Yes $\quad$ No

If YES to $b$ ):

Will participants have the opportunity to request that certain responses remain non-attributable?

$\square$ Yes $\quad \square$ No

7.3 Limitations on anonymity and confidentiality: If the researcher(s) anticipate a situation that may result in a duty to report the names of participants and other relevant information please describe those circumstances here.

\section{Section 8: Informed Consent}

8.1 Describe the procedures for obtaining informed consent for each part of the research project.

All participants will be asked to review information provided about the project and sign a document of informed consent that indicates they have read and agreed to the interview. 
8.2 Participant withdrawal: (Please explain the procedures for participants to withdraw from the research study.)

Any participant may withdraw from the study at any time, without giving a reason, by stating this wish to me. If a participant decides to withdraw, they will have their data destroyed immediately.

\subsection{Future use of data:}

The research information forms ( see Appendix 3) clearly outlines all possible future uses of the collected data. The data collected will be destroyed once the project is complete.

8.4 Append the required informed consent form (For Carleton researchers all consent forms must be on Carleton University letterhead.)

\section{Appendix 3}

\section{Section 9: Data Collection, Storage and Dissemination}

9.1 On-line survey section only: $x$ Not applicable

Is the host survey company Canadian? $\square$ Yes $\quad \square$ No

If No, in what country will the host data be stored?

Describe the process for transferring the data from the host server to you and verification that the host server is no longer in possession of the data.

\subsection{Audio, video recording or photographs}

Will the research participants be audio, video recorded or photographed? $\mathrm{x}$ Yes

No

If Yes: Please describe how the recordings or photographs will be used. If they are to be used in any presentations of the research participants must be made aware of this and consent to their use. If recordings or photographs are to be destroyed explain how and when this will occur.

The interviews will be audio recorded for the purposes of data collection only. The audio recordings will be encrypted using Truecrypt, an open source encryption program, and will be maintained in a password protected folder on a secure server (Carleton University) with a coded non-identifying label ( eg. SB 1001). These recordings will be deleted once transcription is complete.

\subsection{Translation and transcription of data:}


Will the research project require the services of a translator or transcriber? (Check No if researcher is translator) $\quad \square$ Yes $\quad x$ No

If Yes: please describe what steps will be taken to ensure the privacy and confidentiality of the participants. Please provide a copy of the confidentiality agreement for the translator or transcriber.

9.4 Storage of data: (Explain how the data will be stored during the course of the study. If the data will be used in future studies explain how it will be stored.)

During the course of the study, the data will be maintained in a password protected folder on the Carleton University server. The data will be encrypted, using Truecrypt, an open source encryption tool that has worked well for other research studies.

9.5 Access to data: (Explain who will have access to the data during the course of the study.)

Only me, the researcher.

9.6 Disposition of Data (After analysis and completion of report)

Data will be:

Returned to participants (Describe in what format the data will be returned and approximate date it will be returned to participants.)

x Destroyed (Describe how the data will be destroyed and approximate date of destruction. This includes all audio tapes, digital recordings, videos and photographs.)

The digital recordings will be retained on the Carleton University server until the transcripts are received then will be eliminated entirely, using the directions of IT services. No paper copies of the transcripts will be maintained. The code for the transcripts will be kept in an unlabelled notebook in a locked drawer in the researchers' office. The transcripts will be destroyed in May 2014 when the project is meant to end.

Data to be stored and used in future projects (Describe how the data will be stored; including the storage format and how it may be used in future research projects. NOTE: the data can only be used in the same or related projects.)

9.7 Dissemination: (Check all that apply) 
x Academic journals

$\square \operatorname{Book}(\mathrm{s})$

$x$ Conferences

$\square$ Final report to organization
Web site/publication (open to the public)

Workshops

Classroom presentations/exercises

$x$ Final report to participants, upon request

\section{Section 10: Research Instrument}

Append a copy of all research instruments for the project. This includes questionnaires, interview guides, sample questions, written tests and assignments, descriptions of apparatus and

equipments.

Appendix 1 Interview Schedule guide for key informants (attendants, ex-attendants)

Appendix 2 Interview Schedule guide for key informants (ex-clients) 
Sub- Appendix F: Ethics Clearance Form 


\section{Carleton \\ U N I VERS I T Y}

Canada's Capital University
Carleton University Research Office Research Ethics Board

1325 Dunton Tower

1125 Colonel By Drive

Ottawa, ON K1S $5 B 6$ Canada

Tel: $613-520-2517$

ethicsalacarletion.ce

\section{Ethics Clearance Form}

This is to certify that the Carleton University Research Ethics Board has examined the application for ethical clearance. The REB found the research project to meet appropriate ethical standards as outlined in the Tri-Council Policy Statement: Ethical Conduct for Research Involving Humans, $2^{\text {nd }}$ edition and, the Carleton University Policies and Procedures for the Ethical Conduct of Research.

$X$ New clearance

- Renewal of original clearance
Original date of clearance:
Date of clearance

Researcher

Department

Supervisor

Project number

Title of project
5 December 2013

Graham G. Macdonald, Master's student

Institute of Political Economy

Prof. Susan Braedley, School of Social Work

100536

The political ecology of independence: Ethnography of an independent living program

\section{Clearance expires: 31 May 2014}

\section{All researchers are governed by the following conditions:}

Annual Status Report: You are required to submit an Annual Status Report to either renew clearance or close the file. Failure to submit the Annual Status Report will result in the immediate suspension of the project. Funded projects will have accounts suspended until the report is submitted and approved.

Changes to the project: Any changes to the project must be submitted to the Carleton University Research Ethics Board for approval. All changes must be approved prior to the continuance of the research.

Adverse events: Should any participant suffer adversely from their participation in the project you are required to report the matter to the Carleton University Research Ethics Board. You must submit a written record of the event and indicate what steps you have taken to resolve the situation.

Suspension or termination of clearance: Failure to conduct the research in accordance with the principles of the Tri-Council Policy Statement: Ethical Conduct for Research Involving Humans, $2^{\text {nd }}$ edition and the Carleton University Policies and Procedures for the Ethical Conduct of Research may result in the suspension or termination of the research project.

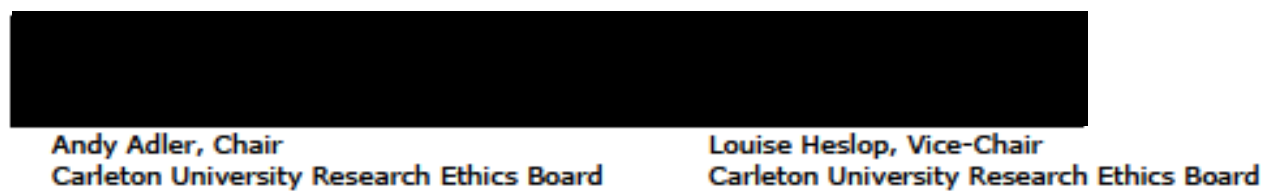

